\title{
Coarse-Grained Effective Action and Renormalization Group Theory in Semiclassical Gravity and Cosmology
}

\author{
E. A. Calzetta* \\ Departamento de Física and IAFE, Facultad de Ciencias Exactas y Naturales \\ Universidad de Buenos Aires- Ciudad Universitaria, Pabellón I \\ 1428 Buenos Aires, Argentina \\ B. L. $\mathrm{Hu}^{\dagger}$ \\ Department of Physics, University of Maryland \\ College Park, MD 20742, USA \\ Francisco D. Mazzitelli $\ddagger$ \\ Departamento de Física \\ Facultad de Ciencias Exactas y Naturales \\ Universidad de Buenos Aires- Ciudad Universitaria, Pabellón I \\ 1428 Buenos Aires, Argentina
}

(October 22, 2018)

In this report we introduce the basic techniques (of the closed-time-path coarse-grained effective action) and ideas (scaling, coarse-graining and backreaction) behind the treatment of quantum processes in dynamical background spacetimes and fields. We show how they are useful for the construction of renormalization group (RG) theories for studying these nonequilibrium processes and discuss the underlying issues. Examples are drawn from quantum field processes in an inflationary universe, semiclassical cosmology and stochastic gravity. In Part I we begin by establishing a relation between scaling and inflation, and show how eternal inflation (where the scale factor of the universe grows exponentially) can be treated as static critical phenomena, while a 'slow-roll' or power-law inflation can be treated as dynamical critical phenomena. In Part II we introduce the key concepts in open systems and discuss the relation of coarse-graining and backreaction. We recount how the (in-out, or Schwinger-DeWitt) coarse-grained effective action (CGEA) devised by Hu and Zhang can be used to treat some aspects of the effects of the environment on the system. This is illustrated by the stochastic inflation model where quantum fluctuations appearing as noise backreact on the inflaton field. We show how RG techniques can be usefully applied to obtain the running of coupling constants in the inflaton field, followed by a discussion of the cosmological and theoretical implications. In Part III we present the Closed-Time-Path (CTP, in-in, or Schwinger-Keldysh) CGEA introduced by $\mathrm{Hu}$ and Sinha. We show how to calculate perturbatively the CTP CGEA for the $\lambda \Phi^{4}$ model. We mention how it is useful for calculating the backreaction of environmental fields on the system field (e.g. light on heavy, fast on slow) or one sector of a field on another (e.g. high momentum modes on low, inhomogeneous modes on homogeneous), and problems in other areas of physics where this method can be usefully applied. This is followed by an introduction to the influence functional in the (Feynman-Vernon) formulation of quantum open systems, illustrated by the quantum Brownian motion models. We show its relation to the CTP CGEA, and indicate how to identify the noise and dissipation kernels therein. We derive the master and Langevin equations for interacting quantum fields, represented in the works of Lombardo and Mazzitelli and indicate how they can be applied to the problem of coarse-graining, decoherence and structure formation in de Sitter universe. We perform a nonperturbative evaluation of the CTP CGEA and show how to derive the renormalization group equations under an adiabatic approximation adopted for the modes by Dalvit and Mazzitelli. We assert that this approximation is incomplete as the effect of noise is suppressed. We then discuss why noise is expected in the RG equations for nonequilibrium processes. In Part IV, following

\footnotetext{
*Email: calzetta@df.uba.ar

${ }^{\dagger}$ Email: hub@physics.umd.edu

${ }^{\ddagger}$ Email: fmazzi@df.uba.ar
} 
Lombardo and Mazzitelli, we use the RG equations to derive the Einstein-Langevin equation in stochastic semiclassical gravity. As an example, we calculate the quantum correction to the Newtonian potential. We end with a discussion on why a stochastic component of RG equations is expected for nonequilibrium processes.

\section{AIM AND SCOPE}

We discuss how the concepts of open systems and the techniques of the coarse-grained effective action and influence functionals can be applied to nonequilibrium quantum processes in the early universe and in semiclassical gravity, leading to renormalization group $(R G)$ theories for the description of the interaction dynamics of these theories.

Our wish is that by examining a sample class of problems of fully dynamic nature -as different from equilibrium (finite temperature) or near-equilibrium (linear response theory) - we can lay out the issues and approaches useful for the construction of a RG theory for nonequilibrium (NEq) processes involving quantum fields.

Description of phase transitions involves a scale that measures the behavior of the order parameter field in the critical region. The energetics of the system is characterized by its quantum dynamical and statistical mechanical properties. At the heart of NEq statistical mechanics is the interplay of the dynamical scales of the system (from the time-dependent order-parameter field) and some background (e.g., all physical processes in an expanding universe are measured against the time-dependent metric function such as the scale factor $a(t)$ ). In equilibrium treatment, this is usually captured by a finite temperature effective potential. But since the order parameter field is generally time dependent, and there may not be a thermal equilibrium environment present in these dynamical processes, one should really be working with an effective action or a free energy density functional. Unlike scattering problems commonly found in particle physics where one can determine the transition amplitude between the in and out states based on the in-out or Schwinger-DeWitt effective actions, in evolutionary problems frequently encountered in statistical mechanics, the development of the expectation value (of an operator associated with some physical variable, such as the energy momentum tensor) need be obtained from the in-in or Schwinger-Keldysh effective actions. When open system concepts like coarse-graining and backreaction of the system and environment are applied, the CTP CGEA or the influence action are more appropriate. (For the development and application of these ideas applied to problems in gravitation and cosmology, see, e.g., [1] 8 ).

This paper is in the nature of a report rather than a review - in that we will present or develop only those works which are useful for the construction of the conceptual and technical frameworks to treat this broad class of problems, taking specific examples from semiclassical gravity and inflationary cosmology as illustrations.

RG concepts and techniques have been used in other areas of gravitation and cosmology. We mention some representative works:

1) RG in gravitational collapse: The use of universality and scaling ideas in classical gravitational collapse first discussed by Choptuik [9] has grown since then into an interesting area of classical graviational research. Fractal structure and scaling laws in a self-gravitating gas have been investigated by de Vega, Sanchez and Combes 10

2) RG in quantum field theory in curved spacetime: notable work since the 80 's by Calzetta, Hu, O'Connor, Jack, Parker, Toms and others as well as the Tomsk group can be found in the book of Buchbinder, Odintsov and Shapiro [1]. For more recent works see 12 and [13].

3) Scaling in quantum gravity has been pursued by Ambjorn in a simplicial gravity approach [14 and by Antoniadis, Mazur and Mottola [15, to mention just a few notable avenues of inquiry.

The organization of this paper is as follows: In Sec. 2 we first give a descriptive summary of the nature of the problems encountered in phase transitions in the early universe, focussing on the inflationary cosmology. We distinguish the case of eternal inflation from that of slow roll and indicate why they can be viewed as static and dynamic critical phenomena respectively. In Sec. 3 we begin a discussion of the relation of scaling, coarse-graining and backreaction with the example of stochastic inflation, thus bringing out the basic concepts of open systems. In Sec. 4 we introduce the ('in-out') coarse-grained effective action (CGEA) for a $\lambda \phi^{4}$ field to incorporate the backreaction effect of the short wavelength modes viewed as the environment, on the long wavelength modes of the system. In Sec. 5 we perform a rescaling of the modes and the field in the spirit of RG transformations and derive the correspondng $R G$ equations for the coupling constants 
of the system field. We briefly discuss the theoretical and cosmological implications. In Sec. 6, we present the 'in-in' or closed-time-path (CTP) CGEA and show how it is useful for the derivation of real and causal dynamical equations for expectation values of operators of the system fields with backreaction from the environment field. We carry out a perturbative evaluation of the CTP CGEA and show how it could be useful for the consideration of backreaction of environment field (modes) on the system field (modes). In Sec. 7 we introduce the influence functional formulation via the quantum Brownian motion model and show its relation with the CTP CGEA. In this process we obtain the master and semiclassical Langevin equations for interacting quantum fields. We show how the noise kernels can be identified in this equation and how their behavior can be used as a measure of decoherence. In Sec. 8 we carry out a nonperturbative evaluation of the CTP CGEA, and derive the RG equations under different approximations. We indicate how our realtime CTP Coarse-Grained Effective Action (CGEA) approach differs from the Euclidean Averaged Effective Action (EAEA) approach. In Sec. 9 we use RG theory to derive the Einstein-Langevin equation in stochastic semiclassical gravity. In Sec. 10 we show how the RG equations change the Newtonian potential. In Sec. 11, following a short summary, we discuss the salient features of RG theory for nonequilibrium processes and argue why there is a stochastic component in the RG equations for such systems.

Those readers who only wish to learn the methodology which underlies nonequilibrium quantum field processes, but have no special interest in the specifics of such processes in gravitation and cosmology, can do just read Sections 4, 6, 8 and 11, from which they will be able to apply the methods to their own areas of research (e.g., nuclear/particle, atomic/optical physics).

\section{PART ONE: Scaling and Inflation}

\section{PHASE TRANSITIONS IN THE EARLY UNIVERSE}

We use phase transitions in the early universe (from the Planck time to the GUT time, or even at the later electroweak era) as working examples to illustrate the basic issues and methods involved. This is because phase transitions in the early universe are mediated by and involve many NEq processes of fundamental interest (e.g., nucleation, spinodal decomposition, particle creation, decoherence) that lead to many important physical consequences such as entropy generation and structure formation. We will only dwell on those aspects which illustrate the applications of the RG theory and suggest its extension to dynamical processes. The development of a RG theory for NEq processes here - from the construction of the CGEA to the derivation of the RG equations - is based on the concepts (scaling, coarse-graining, backreaction) and techniques (CTPEA and influence functional) of nonequilibrium mechanics and quantum field theory. These ideas and techniques are generally applicable also to problems outside of gravitation and cosmology, such as the quark-gluon plasma and atoms interacting with a Bose-Einstein condensate. On the other hand this parallel presentation of RG theory in the context of NEq processes may be helpful to cosmologists who wish to find a more solid theoretical anchor for the discussion of phase transitions in the early universe.

\section{A. Effective Action for Dynamic Order-Parameter Fields}

As noted earlier knowledge of the exact form of the CTP CGEA holds the key to a complete description of a phase transition. One can deduce not only the qualitative features (first or second order) but also the quantitative details (mechanisms and processes). Therefore the construction of the effective action for

the order parameter field in different cosmological spacetimes is usually the necessary first step towards a description of phase transitions in the very early universe.

For the purpose of illustration it is useful to establish the connection with ideas of RG theory at the outset. The two themes established in [4,5], which we follow here are: 1) Eternal inflation can be described equivalently as an exponential scale transformation, thus rendering this special class of dynamics as effectively static. 2) The class of 'slow-roll' inflation can be treated as a dynamical perturbation off the effectively static 
class of exponential inflation and be understood as a dynamical critical phenomenon in cosmology. I

Let us concentrate on situations where the order parameter field changes either with space or time. (A familiar example in condensed matter physics is anisotropic superconductivity where one can use a gradient expansion in the Landau-Ginzburg-Wilson effective potential to account for the differences coming from the next-to-nearest neighbor interactions.) For cosmological problems, it is the time-dependence of the background field which one needs to deal with. 2 Thus for a realistic description of many inflationary transitions one needs to treat the case of a dynamical field and a nonflat or even quasi-static potential. The form of the potential and the metric of the background spacetime together determine the behavior of the scalar field in the Laplace-Beltrami equation, but the field in turn provides the source of the Einstein equation which determines the behavior of the background spacetime metric. Hence they ought to be solved self-consistently. (One usually considers only the homogeneous mode of the scalar field for the dynamics of inflation and the inhomogeneous modes of quantum fluctuations for processes like structure formation.)

At the classical level, the wave equation for the background scalar field (assumed homogeneous) with self-interaction potential $V(\phi)$ in a spatially-flat Robertson-Walker (RW) spacetime is given by

$$
\ddot{\phi}+3 H(t) \dot{\phi}+V^{\prime}(\phi)=0
$$

and the Einstein equations read

$$
\dot{H}+3 H^{2}=8 \pi G V(\phi), \quad \dot{H}=-4 \pi G \dot{\phi}^{2}
$$

where $H(t) \equiv \dot{a} / a$ is the Hubble rate, a dot denotes derivative with respect to cosmic time $t$, and a prime denotes a derivative taken with respect to its argument. A trivial but important solution to these equations is obtained by assuming that $V(\phi)=V_{0}=$ constant, $\phi=\phi_{0}=$ constant and $H=H_{0}=$ constant, which is the de Sitter universe $a=e^{H_{0} t}$ with a constant field. A less trivial but useful solution is the so-called 'power-law' inflation models [22], with an exponential potential and a slowly-varying inflaton field. One can carry out a derivative expansion of the background field to obtain a quasi-local effective action for the description of such classes of spacetimes and fields. (An example of 'slow-roll' transition.)

\footnotetext{
${ }^{1} \mathrm{~A}$ comment on the meaning of the term 'critical dynamics' as used in the context of cosmological phase transitions is in order. By it we refer to studies of phase transitions mediated by a time-dependent order parameter field in contrast to static critical phenomena where the order-parameter field is constant in time. We are using this term in a general sense, not necessarily referring to the specific conditions of critical phenomena as discussed in condensed matter systems [16]. For example, critical phenomena usually deals with the change of the order parameter field near the critical point as a function of temperature. In cosmology, temperature $T$ is a parameter usually (e.g. under the assumption of adiabatic expansion) tied in with the scale factor $a$ and does not play the same role as in critical phenomena. In the new inflationary scenario, the critical temperature $T_{c}$ is defined as the temperature at which a global ground state (the true vacuum) first appears. The stage when vacuum energy begins to dominate and inflation starts is the beginning of the phase transition. The stage when the system begins to enter the true vacuum and reheat can be regarded for practical purposes as the end of the phase transition. Throughout the process of inflation the system is in a 'critical' state. The progression of a cosmological phase transition is measured not by temperature, but by the change of field configurations in time. Criticality corresponds to the physical condition that the correlation length $\xi=m_{e f f}^{-1} \rightarrow \infty$, or $m_{\text {eff }}^{2}=d^{2} V /\left.d \phi^{2}\right|_{\phi=0} \rightarrow 0$ (which may or may not be possible). In critical dynamics studies of condensed matter systems one usually analyses the time-dependent Landau-Ginzburg equation, with a noise term representing the effect of a thermal bath, and studies how the system (order-parameter field) settles into equilibrium as it approaches the critical point. We are not concerned with the corresponding cosmological problem here. An attempt to describe this aspect of the inflationary transition was made in [17]. See also [18].

${ }^{2}$ Strictly speaking, phase transition studies usually carried out assuming a constant field in the de Sitter universe are unrealistic, in that they only address the situation after the universe has entered the inflationary stage and inflates indefinitely. This model cannot be used to answer questions raised concerning the likelihood that the universe will still inflate if it had started from a more general, less symmetric initial state, such as the mixmaster universe 1921. Nor can one use this model to study the actual process of phase transition (e.g., slow roll-over), and the problem of exit (graceful or not) to the 'true' Friedmann phase. To do this, as is well-known, one usually assumes that the potential is not exactly flat, but has a downward slope which enables the inflaton field to gradually (so as to give sufficient inflation) settle into a global ground state. The cosmological solution is, of course, no longer a de Sitter universe.
} 


\section{B. Inflation as Scaling: Static Critical Phenomena}

This idea arose from the work of $\mathrm{Hu}$ and Zhang [4] on coarse-graining and backreaction in stochastic inflation. In trying to compare the inflationary universe with phase transitions in the Landau-Ginzburg model, using a $\lambda \phi^{4}$ field as example, they realized that the exponential expansion of the scale factor can be viewed as the system undergoing a Kadanoff-Migdal scale transformation [23] (this is explained in detail in Sec. 3.B). In other words, time in this case plays the role of a scaling parameter. It does not have to be viewed as a dynamical parameter. Thus for this special class of expansion, the dynamics of spacetime can be replaced equivalently by a scaling transformation. In so doing one renders eternal inflation into a static setting. By contrast, the larger class of power-law expansion $a=t^{\gamma}$ does not possess this scaling property. A useful parameter which marks the difference between these two classes of dynamics is $\zeta=|\dot{H}| / H^{2}=\ddot{\alpha} / \dot{\alpha}^{2}$, where $\alpha \equiv \ln a$, which can be regarded as a 'nonadiabaticity parameter' of dynamics: the de Sitter exponential behavior with $\zeta=0$ is 'static', the slow-roll with small $\zeta<<1$ is 'adiabatic', while the RW low-power-law with $\zeta \approx 1$ is 'nonadiabatic'.

\section{Quasi-Static Field, 'Slow-Roll' as Dynamical Critical Phenomena}

The effective potential $V(\phi)$ gives a well defined description of phase transition only for a constant background (order-parameter) field. If the order-parameter field is dynamic, the effective potential is ill- defined and a host of problems will arise. Indeed, the very meaning of phase transition can become questionable. This is because as the field changes the effective action functional changes, and the location of the minima changes also. The notion of symmetry breaking and restoration is meaningful only when there exist well-defined global and local minima which do not change much in the time scale of the phase transition. Changing background fields will also engender particle creation, which affects the nature and energetics of phase transition as well. Therefore, in the context of phase transitions involving dynamic fields, short of creating a new framework, one can at best discuss the problem in a perturbative sense, where the background field is nearly constant (quasi-static), so that an effective quasi-potential can still be defined [24,25]. An effective Lagrangian for a slowly-varying background field can be obtained by carrying out a quasilocal expansion in derivatives of the field, the leading term being the effective potential [26].

$$
\mathcal{L}=\mathcal{L}\left(\phi, \partial_{\mu} \phi, \partial_{\mu} \partial_{\nu} \phi, \ldots\right)
$$

One can use this method to derive effective quasi-potentials for scalar fields in flat space (for an example of its application to electroweak finite temperature transition see, e.g., Moss et al [27]) or (in conformal time) for the conformally-flat Robertson-Walker spacetimes. This is useful for studying cosmological phase transitions where the background spacetime changes only gradually, as in the Friedmann (low-power law) solutions $a=t^{\gamma}, \gamma<1$. (For a description see [25].) However, for the inflationary universe where the scale factor undergoes rapid expansion following either an exponential $a=e^{H t}$ or a high power-law behavior, the quasilocal expansion which assumes that the background field varies slowly is usually inadequate.

Using the conceptual framework introduced above, one can understand why the particular subclass of high-power-law expansion associated with an exponential potential can hence be viewed as quasi-static. It is in this context that one can introduce the quasi-'static' approximation to derive the effective action for scalar fields to depict this more realistic 'slow-roll' inflation, now carried out as a quasilocal perturbation from the de Sitter space, which is viewed as effectively static. For slowly-varying background fields one can use the method of derivative expansion to derive the quasilocal effective Lagrangian. Usually this makes sense only for static (or conformally-static, like the RW) spacetimes. However, one can view the special class of exponential expansion as effectively 'static'. This can be understood with the ideas of 'dynamical finite size effect' [28] and implemented by treating inflation as 'scaling' transformations [- The 'slow roll-over' type of phase transition used in many inflationary models can be viewed as a quasi-static case, and derived as a dynamic perturbation from the de Sitter universe [5]. This view reveals a close analogy of this case with dynamical critical phenomena where the scaling parameter $s$ plays the role of inflation and the time parameter $t$ measures how much the system departs from the exponential expansion solution. The main points proposed in [5] can be summarized by the following schematic diagram:

Constant Field in Static or SCALING Exponential Expansion $a=e^{H t}$ Conformally Static Spacetimes — > 'Eternal Inflation' 
(Finite Size Effect)

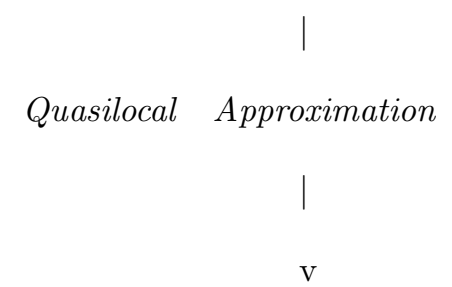

(Dynamical Finite Size Effect)

Derivative Expansion

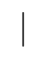

V

Slowly Varying Field in

High Power-Law expansion $a=t^{\gamma}$

RW Universe (Low Power-Law)

$->$ 'Slow Roll-Over'

The inadequacy of the finite temperature effective potential for the description of phase transitions in the early universe was what motivated us to look for more general methods useful for dynamic and nonequilibrium processes, especially involving quantum fields. We will trace out this pathway with two themes, one for the exposition of methods to treat NEq processes, and the other for the development of RG theories for such processes. This will lead us from the effective action to the influence functional methods, and for RG theory, from CTP CGEA to RG equations.

It is better to start with a physical example to motivate, so we first discuss a simple conceptual point which will enable us to see inflation in the light of scaling. 3. $^{\text {. }}$

\section{COARSE-GRAINING, SCALING AND INFLATION}

\section{A. Inflation}

Consider a massive $(\mathrm{m})$, self-coupled $(\lambda)$ scalar field $\phi(\vec{x}, t)$ in a background spacetime with action

$$
S[\phi]=\int d^{4} x \sqrt{-g}\left[-\frac{1}{2} \phi \square \phi-\frac{1}{2}\left(m^{2}+\xi R\right) \phi^{2}-\frac{1}{4 !} \lambda \phi^{4}\right]
$$

where $\square=\frac{1}{\sqrt{-g}} \frac{\partial}{\partial x^{\mu}} g^{\mu \nu} \sqrt{-g} \frac{\partial}{\partial x^{\nu}}$ is the Laplace-Beltrami operator and $\xi=0, \frac{1}{6}$ correspond respectively to minimal and conformal coupling of the field with the background spacetime with scalar curvature $R$. We will consider Robertson-Walker (RW) and de Sitter (DS) spacetimes with line element

$$
d s^{2}=d t^{2}-a^{2} d \Omega^{2}
$$

where $d \Omega^{2}$ is that of the 3 -space $\left(d \Omega^{2}=d \vec{x}^{2}\right.$ for spatially flat). We restrict our attention here only to the spatially homogeneous and isotropic RW, or the spacetime- homogeneous and isotropic DS cases, where a single isotropic scaling parameter is applicable. For the RW universe

$$
a(t)=t^{\gamma},
$$

where $\gamma=1 / 2$ for a radiation-dominated source and $\gamma=2 / 3$ for a pressureless dust source. For a de Sitter universe (in the spatially-flat RW coordinatization)

$$
a(t)=\exp (H t) .
$$

Spatially homogeneous but anisotropic spacetimes are more complicated as they require different scaling parameters in different directions.

In a spatially-homogeneous spacetime, the scalar wave function $\Phi(\vec{x}, t)$

\footnotetext{
${ }^{3}$ Sec. 3, 4, 5 are excerpted from Lectures I and II of [ [4]
} 


$$
\Phi(\vec{x}, t)=\sum \phi_{k}(t) u_{k}(\vec{x})
$$

is the product of a function of time $\phi_{k}(t)$ and a function of space $u_{k}(x)\left[=e^{i \vec{k} \cdot \vec{x}}\right.$ for spatially-flat, and $=Y_{l m n}(\chi, \theta, \phi), k=(l, m, n)$ for spatially-closed cases.] The wave equation for the time-dependent amplitude function $\phi_{k}(t)$ associated with the kth mode of a scalar field $\Phi(\vec{x}, t)$ with self-interaction potential $V(\Phi)$ in a RW spatially-flat spacetime is given by

$$
\frac{d^{2} \phi_{k}}{d t^{2}}+3 H(t) \frac{d \phi_{k}}{d t}+\left(\frac{k^{2}}{a^{2}}+m^{2}+\xi R\right) \phi_{k}+V_{k}^{\prime}(\phi)=0
$$

where $H(t) \equiv \dot{a} / a=\dot{\alpha}$, or $\alpha=\ln a=\int H d t$. (Here a dot denotes derivative with respect to cosmic time $t$ ).

The solution to III.5 depends on $V^{\prime}(\phi)=d V / d \phi$. For inflation we are interested in cases where i) $V^{\prime}(\phi)<<3 H \dot{\phi}$. This would correspond to considering only the relatively flat portion of the potential, where inflation takes place for an extended period of time (usually assumed $\Delta \alpha=\int_{t 0}^{t_{1}} H d t>68 e$-folding time to engender sufficient entropy corresponding to the observed universe). In new inflation, this corresponds to assuming a flat plateau; and in chaotic inflation, a gradual downward slope. In the realistic de Sitter case, the order parameter field $\phi$ changes very slowly in this inflation regime (slow roll-over), its rate controlled by $3 H d \phi / d t$, which can be regarded as a viscous term in the dynamics. One can safely also assume that ii) $\ddot{\phi}<<3 H \dot{\phi}$ in this regime. (By contrast the $\ddot{\phi}$ term dominates the dynamics in the reheating regime). A further simplification is to assume that $\phi=$ constant in this regime throughout, corresponding physically to eternal inflation, i.e., $a=e^{H t}$ for all time. This idealized case is of course unphysical in that the universe will never roll-over (in new and chaotic inflation), or tunnel (in old inflation) to the true vacuum. Nevertheless it captures the salient features of inflation. We shall consider this idealized case in detail here in the light of static scaling. In summary one can distinguish the following cases:

a)Minkowski field theory (static) $\quad \mathrm{H}=0$

b)Eternal inflation (stationary) $\quad \mathrm{H}=$ constant, $\dot{\phi}=0$

c) Slow-roll inflation (quasi-stationary) $\mathrm{H} \simeq$ constant, $\dot{\phi} \neq 0, \ddot{\phi}=0$

d)Realistic inflation (dynamic) $\quad \mathrm{H} \simeq$ constant, $\dot{\phi} \neq 0, \ddot{\phi} \neq 0$

In both cases a) and b), the term $3 H \dot{\phi}$ in III.5 is ignored, while in cases b) and c) the term $\ddot{\phi}$ is ignored.

We now discuss the effect of (eternal) inflation on the scalar field. We will show that a scalar field with $\lambda \phi^{4}$ self-interaction in an isotropically expanding spacetime undergoing eternal inflation behaves exactly like the same field in a static spacetime undergoing a scaling transformation. The physics of inflation (we refer specifically to the initial stage only, but not the subsequent slow roll-over nor the reheating stages) can thus be understood in terms of scaling completely.

\section{B. Scaling}

To fix ideas, consider the spatially-flat RW metric Eq.III.2 with $a=$ constant. This is just the Minkowski spacetime. Let us consider an ordered sequence of such static hyperspaces (foliation) with scales $a_{0}, a_{1}, a_{2}$, etc, parameterized by $t_{n}=t_{0}+n \Delta t, n=0,1,2, \ldots$. These spacetimes have the same geometry and topology but differ only in the physical scale in space. One can always redefine the physical scale length $x_{(n)}^{\prime}=a_{n} x$ to render them equivalent. If each copy has scale length magnified by a fixed factor $e^{H \Delta t}$ over the previous one in the sequence, i.e. $a_{n+1} / a_{n}=e^{H \Delta t}$, we get exactly the physical picture as in an eternal inflation. After $n$-iterations i.e. $a_{n} / a_{0}=e^{n(H \Delta t)}$, or, in terms of a continuous parameter $a(t)=a_{0} e^{H t}$. It is important to recognize here that $t$ can be any real parameter not necessarily describing the dynamics. Time in this depiction plays the role of a scaling parameter, and dynamics is nothing other than scaling.

To see this simple point in another light, let us describe a totally different physical situation (which we will see at the end is exactly identical) where scaling plays the dominant role but one would otherwise not be inclined to associate with the physics of inflationary cosmology. This is the Kadanoff-Migdal transformation 23,29] widely applied to condensed matter systems for the study of critical behavior. Consider a 3 -dimensional cubic lattice with lattice length $L$, and an order parameter field $\phi$ describing the magnetization of the system. The Ginsburg-Landau-Wilson Hamiltonian for a Heisenberg magnet bears similarity with the $\lambda \phi^{4}$ theory in a well-known manner [30].

The Kadanoff-Migdal transform is an artificial procedure for relating the microscopic and macroscopic properties of a system based on the existence of scaling properties in the system in the infrared limit. It 
involves taking a certain number of fixed spin sites (e.g. 4 in 2 dimensions, 8 in 3 dimensions) and replacing them by one block spin with adjusted (e.g. doubled bond strength) interaction strength between nearest neighbors. Carrying out this transformation $n$ times leads to a coarse-grained system. If the system (ferromagnet) manifests scaling properties near the critical point, as had been observed before the renormalization group theory was invented for its description, then the resulting rescaled macroscopic system should preserve the same properties as the original microscopic system. The appearance of long range order near the critical point makes this procedure a viable one, permitting enormous simplification while preserving the salient features of the system.

Let us examine a two-dimensional example of this process, sometimes also called decimation. Denote by $a$ the original length and $a_{n}$ the scale length after carrying out the $K-M$ transformation $n$ times. If 4 -spins are combined into 1 block spin in 2 dimensions, $a_{1}=2 a_{0}$, and $a_{n} / a_{0}=2^{n}$. This gives the same result as inflation, except that the scaling factor $s \equiv a_{n+1} / a_{n}$ here is 2 , instead of $s=e^{(H \Delta t)}$. Defining

$$
s \equiv \frac{a_{n+1}}{a_{n}} \equiv e^{\sigma},
$$

$\sigma$ for inflation is $H \Delta t$, a constant, while for decimation in the above example is $\ln 2$. We shall see that it is precisely this scaling property in inflation which imparts all the distinct and desirable physical features, from the scale-invariant Zel'dovich-Harrison perturbation spectrum to the Hawking effect.

While the above real-space scaling explains well the physical idea, the dual transformation in momentum space is easier to calculate. This is the original Wilson-Fisher RG transformation [29] [30]. In momentum space, the lattice spacing acts like an ultraviolet cut off $\Lambda$ of wave numbers $k$ ( $\Lambda=\infty$ for continuous systems). The block spin transformation corresponds to a) eliminating the higher wave number modes (e.g. $k>\Lambda / s$ ) and b) rescaling (e.g., $k \rightarrow k^{\prime}=k s$ ). The real parameter $s>0$ acts in a) like a coarse-graining (cut parameter) and in b) like a rescaling parameter. In our example above, $s=2$. In every iteration, wavemodes with $k>\Lambda / 2$ are integrated out first, then the remaining modes from $0<k<\Lambda / 2$ are rescaled such that the new $k^{\prime}$ space covers the full range with the same $U V$ cutoff. For definiteness, we will refer to these two steps as coarse-graining (separation) and rescaling. Together these two steps constitute the renormalization group transformation.

Thus in this example, at any iteration, wave numbers $\Lambda / s<k<\Lambda$ are being integrated away, and only the lower wave mode sector is kept. For the long wavelength, low $k$ cutoff, we assume the horizon size $H^{-1}$, because those with lower $k$ having wavelengths greater than the horizon size are of no physical significance to the system, at least during the inflationary stage. (Some of these wavelength will reenter the horizon in the reheating phase and influence later events.) In the language of subdynamics one can call the former the environment (irrelevant sector), and the latter the system (relevant sector). The division of these two sectors changes with the order of iteration (in the language of Kadanoff-Migdal transformation) or time-evolution (for inflationary cosmology) of the system. The coupling of the environment with the system is determined by $V(\phi)$. In addition, for a self-interacting field there are low-low and also high-high mode couplings. A useful way to keep track of these interactions, in particular, is by way of the so-called coarse-grained effective action which we will discuss in the next section.

\section{Coarse-graining and Stochastic Inflation}

In the above discussion we have shown how one can transcribe the dynamical process of inflation to the static transformation of scaling. We also showed how the evolution of the universe during inflation can be viewed as a succession of coarse-grainings, with the higher wave number modes cast away and their effect on the remaining (low $k$ )modes accounted for by a coarse-grained effective action. The resulting RG equations describe the flow of the coupling constants. Using this equivalence, we can conceptually (and technically) replace the inflationary expansion process in time by a running of the field towards the infrared regime.

Let us compare the coarse-graining scheme used in the critical phenomena example above (CP, or Case A) with that of an important class of inflationary cosmologies called stochastic inflation (SI, or Case B), because there are close similarities and basic differences. Starobinsky's scheme [31] is to divide a free scalar field into two parts: the system contains long-wavelength modes outside the horizon $\left(p=k / a<\epsilon H, \ell=p^{-1}\right.$ being the physical wavelength and $\epsilon<1$ a small parameter), which are treated classically; and the bath contains quantum fluctuations of wavelengths $\ell$ shorter than the horizon. Thus the bath in stochastic inflation is obtained from the full spectrum by a window function $\theta(k-\epsilon a H)$, and changes at each moment. Many 
authors have claimed that these high $k$ modes with a dynamic cutoff which obey the wave equation (III.5) (with first order or with both first and second order time derivative terms) give rise to a white noise. They then proceed to set up and solve a Langevin equation for the classical field $\phi$ driven by the de Sitter dynamics and with this white noise source. Thus the name stochastic inflation. If

The physical setup of Starobinsky's stochastic inflation differs from the example we used above to illustrate the relation of scaling and inflation in how the system is defined. Technically there exists a simple transformation between coarse-graining in critical phenomena (CP, Case A) and that in stochastic inflation (SI, Case B). Let us explain:

Compare the composition of the system (S) and the environment (E) at the beginning and end of inflation for critical phenomena (Case A) and for stochastic inflation (Case B). For Case B, at the start $t_{0}$, assume $a\left(t_{0}\right)=1$ and $\epsilon=1$ for simplicity. At any later time the system consists of all modes $k<H a$ or $\ell>H^{-1}$. As the universe inflates $a=e^{H t}$, more $k$ modes are being red-shifted out of the horizon into the system. Thus $S$ of stochastic inflation increases (from the $k$ region $H_{0} K$ at $t_{0}$ to $H K$ at $t_{*}^{H}$ to $G K$ at $t_{*}^{G}$, see Fig. 1). The reason why in the stochastic inflationary program the system is taken to consist of modes with wavelengths greater than the horizon $H^{-1}$ in the inflation regime is because these are the modes which would reenter the RW horizon in the radiation-dominated RW phase; and only those higher $\left(k>k_{G}\right)$ modes (here treated as environment ) which reenter the horizon before galaxy formation can physically influence $k_{G}$ in the RW phase. So the system in stochastic inflation is really designed for and chosen by the physics in the later radiation-dominated $R W$ regime, rather than during the inflationary regime. Note that modes with wavelengths greater than the horizon size at the onset of inflation have not yet reentered today's horizon, because in, say, the new inflationary scenario, today's observable universe is believed to be only a small portion $\left(l_{H}\right)$ of a much larger bubble inflated from a fluctuation domain $\left(l_{D}\right)$ at $t_{0}$. The mode $k_{H}$ exits the horizon at time $t_{*}^{H}$. The wave mode $k_{H}$ of physical wavelength $\ell_{H}$ (today's Hubble radius) which is just entering the RW horizon today [and which disappeared outside the de Sitter horizon earlier than any observable physical scale $\left(H R_{H} H_{\text {now }}\right.$ in Fig. 1)] forms the upper limit of $\ell_{\text {phys }}$. Of course more wave modes with $\ell>\ell_{H}$ will be entering into the RW horizon tomorrow. These modes have not yet come into contact with our universe so they should be considered as unphysical by today's observers. However, they had interacted previously in the de Sitter phase at times $t<t_{*}^{H}$ with all other k modes $<k_{H_{0}}$ within the de Sitter horizon. In the inflationary phase, it is this interaction which one should address.

By contrast, in critical phenomema (Case A) depicted earlier, both the system and the bath are contained in the horizon throughout the period of inflation from $t_{0}$ to $t_{1}$ (the $\mathrm{OLH}_{1}$ portion in Fig. 1). $H^{-1}$ is the lower (IR) cutoff. The partition is defined by the incremental fraction $s=e^{H \Delta t}=a(t+\Delta t) / a(t)$ of $k$ modes slipping into the bath at each moment $\Delta t$ (or each iteration) by coarse-graining. For example, one possible partition $k_{L}$ (point $L$ in Fig.1) is determined by the last wave mode which left the horizon at the end of inflation at $t_{1}$. All modes with $k>k_{L}$ never made it out and always stayed inside of the DS horizon. These will be the common "bath" to all systems at all times in the DS phase. Another choice more geared towards the galaxy formation problem is defined by the $k$ modes of interest (e.g. galaxy scale $k_{G}$ ) and made up of $k<k_{G}$. The environment then contains modes $k>k_{G}$, including the common portion $k>k_{L}$. In this designati on we make sure that: 1) The system consists of all scales within today's Hubble radius (RW horizon). 2) Their interactions in the inflationary phase with the higher and lower modes are incorporated. 3) The environment is always within the de Sitter horizon. We are interested in two effects: 1) The effect of the higher $k$ modes field $\phi_{>}$on the system field made up of lower $\mathrm{k}$ modes $\phi_{<}$, and 2) How the field parameters (such as mass $m$ and coupling constants $\lambda$ ) change in this process of inflation (or scaling). These are answered respectively by 1) deriving the effective action and the associated effective equations for $\phi_{<}$, and 2) deriving the renormalization group equation for these field parameters.

Despite the difference in the aims of the two programs the coarse-graining technique used in both cases are related in a simple manner. By replacing $s \rightarrow s^{-1}$, or equivalently $(\Delta t) \rightarrow-(\Delta t)$ in Case $\mathrm{A}$, one gets the procedure in Case $B$ for stochastic inflation. This amounts to changing inflation to deflation (time-reversal), or interchanging system and bath. One can also view the difference as representing a passive versus an active

\footnotetext{
${ }^{4}$ Authors of 4 6 62 question how a white noise can be deduced by this dynamic partitioning of a free field. They suggested instead how colored noise can be generated from coarse-graining an interacting field without assuming any dynamic partitioning (see Section VII B). This provides a more general scheme for stochastic inflation, where one can discuss issues like non-Gaussian galaxy distribution from vacuum fluctuations.
} 
view. By this we mean inflating the background (spacetime) has, for a local observer in a scale-invariant system, the same effect as coarse-graining the system in a fixed background.

A figurative description which encapsulates the main ideas in this comparison is the effect of a zoom lens. The physics of a scale-independent system under inflation is equivalent to holding the system static and viewing it through a zoom lens. Here zooming (scaling) replaces dynamics (inflation). Features of an evolving system undergoing inflation (the passive interpretation) can be depicted equally by the magnifying view through a zoom lens (scaling) of a fixed system (the active interpretation). Obviously this relation holds only if the structure is homogeneous and the lens has uniform magnification. (This corresponds to homogeneity in space and uniform scaling.)

In the next section we present an effective action formalism for carrying out the coarse-graining. We shall keep the formalism general and non-specific to the system-environment stipulation so that it can be applied to different situations in a variety of problems.

\section{PART TWO: Coarse-Graining and Renormalization Group}

\section{COARSE-GRAINED EFFECTIVE ACTION}

\section{A. Coarse Graining and Backreaction}

In quantum field theory, when the background field or spacetime dynamics follows some simple discernable (e.g., classical) behavior one commonly performs what is called a background field decomposition,

$$
\phi=\phi_{c}+\phi_{q}
$$

on a (e.g., self-interacting) scalar field $\phi$. One then calculates the effect of the fluctuation field $\phi_{q}$ on the background field $\phi_{c}$ (as well as the background geometry $g_{\mu \nu}$ when necessary) using a loop expansion in the in-out effective action. For many problems such as those encountered in statistical mechanics where one is interested in the detailed behavior of only a part of the overall system (call it the system) interacting with its surrounding (call it the environment), one can decompose the field describing the overall system $\phi$ according to

$$
\phi=\phi_{S}+\phi_{E}
$$

where $\phi_{S}$ denotes the system field and $\phi_{E}$ the environment field. One can calculate the backreaction of the latter on the former with the 'coarse-grained effective action' (CGEA) first introduced by Hu and Zhang (4), where one does not integrate over the quantum fluctuations, as in the conventional definition (SchwingerDeWitt) of effective action in orders of $\hbar$, but rather with respect to a small parameter $\epsilon$ which can generally be expressed as the ratio of two time, length, mass or momentum scales characteristic of the system and environment discrepancy (examples are slow-fast time scale, long-short length scale, light-heavy mass scale or soft-hard momentum scale). In the stochastic inflation scheme [31], one regards the system field as containing only the lower modes and the environmental field as containing the higher modes with the division provided by the event horizon in de Sitter spacetime. In post-inflationary reheating, the quantum (inhomogeneous) fluctuation fields are parametrically amplified by the time-dependent (homogeneous) inflaton (background) field, resulting in particle creation. Its backreaction effect is to damp away the oscillations of the inflaton field and reheat the universe. (See, e.g., [33,34]). Similar processes of particle creation at the Planck time leading to damping of anisotropy of the background spacetime were earlier treated by means of a closed-time-path (CTP), or in-in effective action. (See, e.g., 11). In quantum cosmology one usually makes the so-called 'minisuperspace approximation' of quantizing only the homogeneous universes and ignoring the inhomogeneous ones. The validity of this truncation scheme can be examined as a backreaction problem. One can view the homogeneous and inhomogeneous universes as the low and high lying modes of excitation of spacetime (this notion can be made more precise in terms of gravitational perturbations off a background spacetime as described by the Lifshitz wave operator), treat the former as constituting the system field (minisuperspace) and the latter as the environmental field. The magnitude of the backreaction of the latter on the former will give a measure of the validity of the minisuperspace approximation. This 
approach was taken by $\mathrm{Hu}$ and Sinha who introduced the CTP CGEA [35]. The same idea and method should be applicable to backreaction effects of hard thermal loops on the soft sector in quark-gluon plasma [36.

In the high-low mode division, one can think of the system as the average of the field over a spatial volume $\Lambda_{c}^{-3}, \Lambda_{c}=\frac{\Lambda}{s}$ being the inverse of the critical wavelength that separates the system from the environment. The Euclidean version of this coarse-graining leads to the so-called average effective action (AEA), introduced by Wetterich [37. These two formalisms, Euclidean and real time CGEA, though sharing similar ideas have rather different ranges of applications. Wetterich's average effective action averages the fields over a spacetime Euclidean region, while Hu's [4] coarse-grained effective action averages field over a spatial region. The Euclidean AEA is more suitable for near-equilibrium systems, and has indeed been applied to QCD problems like quark-gluon plasma in a quasi-stationary state, while the real-time CTP CGEA gives causal evolution equations for expectation values of quantum operator and is more suitable for the analysis of dynamical problems. We will invoke the AEA in Section VIII A.

\section{B. 'In-Out' Coarse Grained Effective Action}

We now introduce the in-out version of the CGEA. This is because is pedagogically simpler, and is closer to what one learns from textbooks, i.e., the Schwinger-DeWitt effective action. Functionally it is also adequate for the introduction of ideas on coarse-graining and backreaction to the extent of seeing the running of coupling constants leading to a RG theory. But the effective equation of motion with backreaction obtained from an in-out version contains non real and non causal terms. This was first noticed by DeWitt and Jordan [38,39] and Calzetta and $\mathrm{Hu}$ [1,2] in a study of the backreaction of particle creation on a background spacetime or field. For a correct treatment of the backreaction of the environment on the system one needs the in-in or CTP version, which we will discuss in Sec. 6.

Consider the $\lambda \phi^{4}$ model with action (III.1) in a spatially-flat Robertson-Walker universe. The scale factor $a(t)=e^{\alpha}$ is left unspecified for now. Rather than viewing $a(t)$ as a dynamical function determined from Einstein's equations we will, in the spirit of scaling as explained in an earlier section, regard $a$ as a constant and $t$ as a parameter. Although the space can have different scales $a(t)$ at different times $t$, spacetime remains flat at all times. (One can rescale $\vec{x}$ at different times to make them all equal in value.) Thus we can simply think of doing Minkowski field theory here, leaving $a(t)$ as a parameter while carrying out coarse-graining in the 3-dimensional space. The content of this subsection can thus be used without reference to cosmology (simply set $a(t)=1)$. However we do want to tag the scale factor $a(t)$ along so that later we can perform scaling transformations and discuss inflationary cosmology.

For the RW spacetime, the action (III.1) reads

$$
S[\phi]=\int d^{3} x \int d t a(t)\left[-\frac{1}{2} \phi\left(\hat{d}_{2}+\hat{d}_{1}-\hat{\Delta}+\tilde{m}^{2}\right) \phi-\frac{1}{4 !} \tilde{\lambda} \phi^{4}\right]
$$

where

$$
\hat{d}_{2}=a^{2}(t) \partial^{2} / \partial t^{2}, \quad \hat{d}_{1}=a^{2}(t) 3 H \partial / \partial t, \quad \hat{\Delta}=a^{2}(t) \Delta
$$

are the second and first order time derivative operators and $\Delta$ is the spatial Helmholtz operator respectively. Here $H \equiv \dot{a} / a=\dot{\alpha}$ and

$$
\begin{gathered}
\tilde{m}^{2}(t)=a^{2}(t)\left(m^{2}+\xi R\right) \\
\tilde{\lambda}(t)=a^{2}(t) \lambda
\end{gathered}
$$

are the conformally-related generalized mass and field self-coupling parameter respectively. We will now assume $\hat{d}_{1}=\hat{d}_{2}=0$, i.e., consider the case of eternal inflation. In this 'static' limit, one can introduce the momentum space representation on the spatial section

$$
\phi(\vec{x}, t)=(2 \pi)^{-3 / 2} \int d^{3} k e^{i \vec{k} \cdot \vec{x}} \phi(\vec{k}, t)
$$


Then

$$
\begin{gathered}
S[\phi]=\int d t a(t) \int d^{3} k\left[-\frac{1}{2}\left(k^{2}+\tilde{m}^{2}(t)\right) \phi(\vec{k}) \phi(-\vec{k})\right] \\
+(2 \pi)^{-3} \int d t a(t) \int d^{3} \vec{k}_{1} d^{3} \vec{k}_{2} d^{3} \vec{k}_{3} d^{3} \vec{k}_{4}\left[-\frac{1}{4 !} \tilde{\lambda}(t) \delta\left(\vec{k}_{1}+\vec{k}_{2}+\vec{k}_{3}+\vec{k}_{4}\right)\right. \\
\left.\phi\left(\vec{k}_{1}, t\right) \phi\left(\vec{k}_{2}, t\right) \phi\left(\vec{k}_{3}, t\right) \phi\left(\vec{k}_{4}, t\right)\right]
\end{gathered}
$$

For interacting fields in a static spacetime mode-mixing is manifest. If $\hat{d}_{1} \neq 0$ "time" translation-invariance is lost and there will be frequency mixing. This becomes relevant in the context of critical dynamics, as mentioned in Sec. 2.

Now we separate the field $\phi(x, t)$ into two parts, $\phi_{<}$and $\phi_{>}$, which one can refer to as the system and the environment,

$$
\phi=\phi_{<}+\phi_{>}
$$

We assume that $\phi_{<}$contains the lower $k$ wave modes and $\phi_{>}$the higher $k$ modes and consider these two cases:

Case A (critical phenomena)

$$
\phi_{<}:|\vec{k}|<\Lambda / s, \quad \phi_{>}: \Lambda / s<|\vec{k}|<\Lambda,
$$

Case B (stochastic inflation)

$$
\phi_{<}:|\vec{k}|<\epsilon H a, \quad \phi_{>}:|\vec{k}|>\epsilon H a, \quad \epsilon \approx 1 .
$$

Here $\Lambda$ is the ultraviolet cutoff and $s>1$ is the coarse-graining parameter which gives the fraction of total $k$ modes counted in the environment. The separation of $\phi$ can also be made in other manners, depending on the physical setup of the problem and the questions one asks. The formalism we present here can be easily adapted to other types of decomposition.

$S[\phi]$ in (IV.6) can be written as

$$
\begin{aligned}
& S[\phi]=\int d t a(t) \int d^{3} \vec{k}\left(-\frac{1}{2}\right)\left(\vec{k}^{2}+\tilde{m}^{2}(t) \phi_{<}(\vec{k}, t) \phi_{<}(-\vec{k}, t)\right) \\
& +\int d t a(t) \int d^{3} \vec{q}\left(-\frac{1}{2}\right)\left(\vec{q}^{2}+\tilde{m}^{2}(t)\right) \phi_{>}(\vec{q}, t) \phi_{>}(-\vec{q}, t) \\
& +(2 \pi)^{-3} \int d t a(t) \int d^{3} \overrightarrow{k_{1}} d^{3} \overrightarrow{k_{2}} d^{3} \overrightarrow{k_{3}} d^{3} \overrightarrow{k_{4}}\left(-\frac{1}{4 !} \tilde{\lambda}(t)\right) \\
& \times \phi_{<}\left(\vec{k}_{1}, t\right) \phi_{<}\left(\vec{k}_{2}, t\right) \phi_{<}\left(\vec{k}_{3}, t\right) \phi_{<}\left(\vec{k}_{4}, t\right) \delta\left(\vec{k}_{1}+\vec{k}_{2}+\vec{k}_{3}+\vec{k}_{4}\right) \\
& +(2 \pi)^{-3} \int d t a(t) \int d^{3} \overrightarrow{k_{1}} d^{3} \overrightarrow{k_{2}} d^{3} \overrightarrow{k_{3}} d^{3} \overrightarrow{q_{1}}\left(-\frac{1}{4 !} \tilde{\lambda}(t)\right) \\
& \times 4 \phi_{<}\left(\vec{k}_{1}, t\right) \phi_{<}\left(\vec{k}_{2}, t\right) \phi_{<}\left(\vec{k}_{3}, t\right) \phi_{>}\left(\overrightarrow{q_{1}}, t\right) \delta\left(\vec{k}_{1}+\vec{k}_{2}+\vec{k}_{3}+\vec{q}_{1}\right) \\
& +(2 \pi)^{-3} \int d t a(t) \int d^{3} \overrightarrow{k_{1}} d^{3} \overrightarrow{k_{2}} d^{3} \overrightarrow{q_{1}} d^{3} \overrightarrow{q_{2}}\left(-\frac{1}{4 !} \tilde{\lambda}(t)\right) \\
& \times 6 \phi_{<}\left(\vec{k}_{1}, t\right) \phi_{<}\left(\vec{k}_{2}, t\right) \phi_{>}\left(\vec{q}_{1}, t\right) \phi_{>}\left(\vec{q}_{2}, t\right) \delta\left(\vec{k}_{1}+\vec{k}_{2}+\vec{q}_{1}+\vec{q}_{2}\right) \\
& +(2 \pi)^{-3} \int d t a(t) \int d^{3} \overrightarrow{k_{1}} d^{3} \overrightarrow{q_{1}} d^{3} \overrightarrow{q_{2}} d^{3} \overrightarrow{q_{3}}\left(-\frac{1}{4 !} \tilde{\lambda}(t)\right) \\
& \times 4 \phi_{<}\left(\vec{k}_{1}, t\right) \phi_{>}\left(\vec{q}_{1}, t\right) \phi_{>}\left(\vec{q}_{2}, t\right) \phi_{>}\left(\vec{q}_{3}\right) \delta\left(\vec{k}_{1}+\vec{q}_{1}+\vec{q}_{2}+\vec{q}_{3}\right) \\
& +(2 \pi)^{-3} \int d t a(t) \int d^{3} \overrightarrow{q_{1}} d^{3} \overrightarrow{q_{2}} d^{3} \overrightarrow{q_{3}} d^{3} \overrightarrow{q_{4}}\left(-\frac{1}{4 !} \tilde{\lambda}(t)\right) \\
& \times \phi_{>}\left(\vec{q}_{1}, t\right) \phi_{>}\left(\vec{q}_{2}, t\right) \phi_{>}\left(\vec{q}_{3}, t\right) \phi_{>}\left(\vec{q}_{4}, t\right) \delta\left(\vec{q}_{1}+\vec{q}_{2}+\vec{q}_{3}+\vec{q}_{4}\right)
\end{aligned}
$$


where all integrals of $k$ and $q$ have the limits indicated in (IV.7) or (IV.8) depending on the cases in question. Henceforth we shall use the shorthand notation

$$
\phi_{<}(1) \equiv \phi_{<}\left(\vec{k}_{1}, t\right), \quad \phi_{>}(\tilde{1}) \equiv \phi_{>}\left(\vec{q}_{1}, t\right)
$$

and denote

$$
G_{<}(1)=\left[\vec{k}^{2}+\tilde{m}^{2}(t)-i \epsilon\right]^{-1}, \quad G_{>}(\tilde{1})=\left[\vec{q}^{2}+\tilde{m}^{2}(t)-i \epsilon\right]^{-1} .
$$

We can separate terms in (IV.9) into three groups

$$
S[\phi]=S\left[\phi_{<}\right]+S_{0}\left[\phi_{>}\right]+S_{I}\left[\phi_{<}, \phi_{>}\right]
$$

where

$$
\begin{gathered}
S\left[\phi_{<}\right]=-\frac{1}{2} G_{<}^{-1}(1) \phi_{<}(1) \phi_{<}(-1)-\frac{1}{4 !} \tilde{\lambda}(t) \phi_{<}(1) \phi_{<}(2) \phi_{<}(3) \phi_{<}(4) \delta(1+2+3+4) \\
S_{0}\left[\phi_{>}\right]=-\frac{1}{2} G_{>}^{-1}(\tilde{1}) \phi_{>}(\tilde{1}) \phi_{>}(-\tilde{1})
\end{gathered}
$$

and

$$
\begin{aligned}
S_{I}\left[\phi_{<}, \phi_{>}\right]= & -\frac{1}{4 !} \tilde{\lambda}(t)\left[4 \phi_{<}(1) \phi_{<}(2) \phi_{<}(3) \phi_{>}(\tilde{1}) \delta(1+2+3+\tilde{1})\right. \\
& +6 \phi_{<}(1) \phi_{<}(2) \phi_{>}(\tilde{1}) \phi_{>}(\tilde{2}) \delta(1+2+\tilde{1}+\tilde{2}) \\
& +4 \phi_{<}(1) \phi_{>}(\tilde{1}) \phi_{>}(\tilde{2}) \phi_{>}(\tilde{3}) \delta(1+\tilde{1}+\tilde{2}+\tilde{3}) \\
& \left.+\phi_{>}(\tilde{1}) \phi_{>}(\tilde{2}) \phi_{>}(\tilde{3}) \phi_{>}(\tilde{4}) \delta(\tilde{1}+\tilde{2}+\tilde{3}+\tilde{4})\right] \\
& \equiv S_{1}+S_{2}+S_{3}+S_{4}
\end{aligned}
$$

Here integration over k's and q's with the respective ranges is understood, and we have used the simplified notation of $1, \tilde{1}$ to denote $\vec{k}_{1}, \vec{q}_{1}$, etc. It is easier to use Feynman diagrams for the propagators and vertices corresponding to the two fields $\phi_{<}$and $\phi_{>}$. They are listed in Figure 2.

The functional integral formalism for quantum fields is set up in the usual way. Assume that at $t= \pm \infty$, the interaction is turned off so that the in-vacuum $\left|0_{-}\right\rangle$and the out-vacuum $\left|0_{+}\right\rangle$are defined. The vacuum persistence amplitude on the generating functional $\left\langle 0_{+} \mid 0_{-}\right\rangle$gives the probability amplitude of the field remaining in the vacuum after the interacting system evolves in time.

With the splitting $\phi=\phi_{<}+\phi_{>}$, the Schwinger-DeWitt (in-out) generating functional becomes,

$$
Z[\phi]=N \int D \phi e^{i S[\phi]}=N \int D \phi_{<} \int D \phi_{>} e^{\left\{i S\left[\phi_{<}\right]+S_{0}\left[\phi_{>}\right]+S_{I}\left[\phi_{<}, \phi_{>}\right]\right\}}
$$

Introducing an integral over $\phi_{>}$of $\operatorname{expi\mathrm {S}_{0}}\left[\phi_{>}\right]$as the norm for the functional average in the denominator and changing the constant $N$ to $N^{\prime}$ accordingly, we get

$$
\begin{aligned}
Z[\phi] & =N^{\prime} \int D \phi_{<} e^{i S\left[\phi_{<}\right]}\left(\int D \phi_{>} e^{i S_{0}\left[\phi_{>}\right]} e^{i S_{I}\left[\phi_{<}, \phi_{>}\right]} / \int D \phi_{>} e^{i S_{0}\left[\phi_{>}\right]}\right) \\
& =N^{\prime} \int D \phi_{<} e^{i S\left[\phi_{<}\right]} \times\left\langle e^{i S_{I}\left[\phi_{<}, \phi_{>}\right]}\right\rangle_{\phi_{>}}
\end{aligned}
$$

Here \langle\rangle$_{\phi_{>}}$defines averaging over the $\phi_{>}$field under the free action $S_{0}\left[\phi_{>}\right]$(We write \langle\rangle$_{0}$ interchangeably to emphasize that the averaging is with the free action $S_{0}\left[\phi_{>}\right]$.)

Denoting

$$
\left\langle e^{i S_{I}\left[\phi_{<}, \phi_{>}\right]}\right\rangle_{\phi_{>}}=\exp i \Delta S\left[\phi_{<}\right]
$$

we get 


$$
Z\left[\phi_{<}\right]=N^{\prime} \int D \phi_{<} \exp i S_{e f f}\left[\phi_{<}\right]
$$

The coarse-grained effective action is given by

$$
S_{\text {eff }}\left[\phi_{<}\right]=S\left[\phi_{<}\right]+\Delta S\left[\phi_{<}\right]
$$

where

$$
\Delta S\left[\phi_{<}\right]=-i \ln \left\langle\exp i S_{I}\left[\phi_{<}, \phi_{>}\right]\right\rangle_{\phi_{>}}
$$

If the interaction between the $\phi_{<}$and $\phi_{>}$fields is small (e.g. $\lambda \ll 1$ in a $\lambda \phi^{4}$ theory), one can expand this in a Dyson-Feynman series

$$
\begin{aligned}
\Delta S\left[\phi_{<}\right] & =-i l n \sum_{n=0}^{\infty} \frac{i^{n}}{n !}\left\langle S_{I}^{n}\left[\phi_{<}, \phi_{>}\right]\right\rangle_{\phi_{>}} \\
& =\left\langle S_{I}\left[\phi_{<}, \phi_{>}\right]\right\rangle_{\phi_{>}}+\frac{i}{2}\left[\left\langle S_{I}\left[\phi_{<}, \phi_{>}\right]^{2}\right\rangle_{\phi_{>}}-\left\langle S_{I}\left[\phi_{<}, \phi_{>}\right]\right\rangle_{\phi_{>}}^{2}\right]+\ldots
\end{aligned}
$$

where in the second line terms up to the second order in $S_{I}$ are written out explicitly.

Let us now examine each term in (IV.20) in detail, starting with the first order term $\left\langle S_{I}\left[\phi_{<}, \phi_{>}\right]\right\rangle_{\phi_{>}}$. We see that terms in (IV.13) involve the average of products of $\phi_{>}$fields, but those containing an odd number of fields in the product average to zero. Also any product containing four $\phi_{>}$fields (last term in ([V.13) ) averages out to a quantity which is independent of $\phi_{<}$, i.e., $\left\langle\phi_{>}(\tilde{1})\right\rangle_{0}=\left\langle\phi_{>}(\tilde{1}) \phi_{>}(\tilde{2}) \phi_{>}(\tilde{3})\right\rangle_{0}=0$ and $\left\langle\phi_{>}(\tilde{1}) \phi_{>}(\tilde{2}) \phi_{>}(\tilde{3}) \phi_{>}(\tilde{4})\right\rangle_{0}$ is independent of $\phi_{<}(\vec{k}, t)$. Thus the only contribution to $\left\langle S_{I}\left[\phi_{<}, \phi_{>}\right]\right\rangle_{0}$ is the quadratic product

$$
\left\langle\phi_{>}(\tilde{1}) \phi_{>}(\tilde{2})\right\rangle_{0}=-i a^{-1}(t) G_{>}(\tilde{1}) \delta(\tilde{1}+\tilde{2}) \delta\left(t_{1}-t_{2}\right) .
$$

We denote by $S_{I}\left[\phi_{<}, \phi_{>}\right]_{j}$ the terms containing $j \phi_{>}$fields, i.e. $S_{I 1} \sim \phi_{<} \phi_{<} \phi_{<} \phi_{>}, S_{I 2} \sim \phi_{<} \phi_{<} \phi_{>} \phi_{>}$, etc. In the Feynman diagram depiction (Fig.2c), the averaging over $\phi_{>}$amounts to closing the lines representing the environment field propagators on themselves, and it is easy to see how the above conclusion is reached. Explicitly, the only contributing first order term is

$$
\begin{aligned}
\left\langle S_{I}\left[\phi_{<}, \phi_{>}\right]_{2}\right\rangle_{0}= & (2 \pi)^{-3} \int a(t) d t \int d^{3} \overrightarrow{k_{1}} d^{3} \overrightarrow{k_{2}} \int d^{3} \overrightarrow{q_{1}} d^{3} \overrightarrow{q_{2}} 6\left(-\frac{1}{4 !}\right) a(t) \tilde{\lambda}(t) \\
& \times \phi_{<}\left(\vec{k}_{1}, t\right) \phi_{<}\left(\vec{k}_{2}, t\right)\left\langle\phi_{>}\left(\vec{q}_{1}, t\right) \phi_{>}\left(\vec{q}_{2}, t\right)\right\rangle_{0} \delta\left(\vec{k}_{1}+\vec{k}_{2}+\vec{q}_{1}+\vec{q}_{2}\right)
\end{aligned}
$$

Using (IV.21), we get

$$
\left\langle S_{I}\left[\phi_{<}, \phi_{>}\right]_{2}\right\rangle_{0}=\int a(t) d t \int d^{3} \vec{k}\left(-\frac{1}{2}\right) \phi_{<}(\vec{k}, t) \delta \tilde{m}^{2}(t, s)_{1} \phi_{<}(\vec{k}, t)
$$

where

$$
\delta \tilde{m}^{2}(t, s)_{1}=-\frac{i}{2} \delta(0) \tilde{\lambda}(t) a^{-1}(t)(2 \pi)^{-3} \int d^{3} \vec{q}\left[\vec{q}^{2}+\tilde{m}^{2}(t)-i \epsilon\right]^{-1} .
$$

This is depicted as (1) in Fig. 2c. The subscript 1 on $\delta \tilde{m}^{2}$ denotes that it is the first order correction to $\tilde{m}^{2}$. The second order correction is given by (5) and (6) of Fig. 2c and computed below.

Now we examine the second order terms in (IV.20). The second term in the square bracket $\left\langle S_{I}\left[\phi_{<}, \phi_{>}\right]\right\rangle_{0}^{2}$ gives three non-contributing disconnected graphs. The first term $\left\langle\left(S_{I}\left[\phi_{<}, \phi_{>}\right]\right)^{2}\right\rangle_{0}$ has the following components:

$$
\begin{aligned}
\left\langle S_{I}^{2}\right\rangle_{0}= & \left\langle S_{1}^{2}\right\rangle_{0}+\left\langle S_{2}^{2}\right\rangle_{0}+\left\langle S_{3}^{2}\right\rangle_{0}+\left\langle S_{4}^{2}\right\rangle_{0} \\
& +2\left[\left\langle S_{1} S_{2}\right\rangle_{0}+\left\langle S_{1} S_{3}\right\rangle_{0}+\left\langle S_{1} S_{4}\right\rangle_{0}\right. \\
& \left.+\left\langle S_{2} S_{3}\right\rangle_{0}+\left\langle S_{2} S_{4}\right\rangle_{0}+\left\langle S_{3} S_{4}\right\rangle_{0}\right]
\end{aligned}
$$

where we have written in short 


$$
S_{j}=S_{I}\left[\phi_{<}, \phi_{>}\right]_{j} \quad(j=1,2,3,4)
$$

as in Eq. IV.13). In terms of the Feynman diagrams in Fig. 2c constructed from Fig. 2b, there are four contributing connected diagrams of the second order. They are denoted by (3)-(6) in Fig. 2c. We see that diagram (1) is the first order and (5) (6) are the second order corrections to the mass $\tilde{m}^{2},(4)$ is a second order correction to the coupling constant $\tilde{\lambda}$, while (3) generates $\phi^{6}$ correction.

Adding these contributions together we obtain finally for the coarse-grained effective action up to second order in $\lambda$ :

$$
\begin{aligned}
S_{e f f}\left[\phi_{<}\right]= & \int a(t) d t \int d^{3} \vec{k}\left(-\frac{1}{2}\right) \phi_{<}(\vec{k}, t)\left[k^{2}+\tilde{m}^{2}(t, s)\right] \phi_{<}(-\vec{k}, t) \\
& +\int a(t) d t \int d^{3} \vec{k}_{1} d^{3} \vec{k}_{2} d^{3} \vec{k}_{3} d^{3} \vec{k}_{4}\left(-\frac{1}{4 !}\right) \tilde{\lambda}(t, s,\{\vec{k}\}) \\
& \times \phi_{<}\left(\vec{k}_{1}, t\right) \phi_{<}\left(\vec{k}_{2}, t\right) \phi_{<}\left(\vec{k}_{3}, t\right) \phi_{<}\left(\vec{k}_{4}, t\right) \delta\left(\vec{k}_{1}+\vec{k}_{2}+\vec{k}_{3}+\vec{k}_{4}\right) \\
& +\int a(t) d t \int d^{3} \vec{k}_{1} \ldots d^{3} \vec{k}_{6}\left(-\frac{1}{6 !}\right) \nu_{6}(t, s,\{\vec{k}\}) \\
& \times \phi_{<}\left(\vec{k}_{1}, t\right) \ldots \phi_{<}\left(\vec{k}_{6}, t\right) \delta\left(\vec{k}_{1}+\ldots+\vec{k}_{6}\right) .
\end{aligned}
$$

where the integration range of $\vec{k}_{i}$ is as indicated in (IV.7) and (IV.8): $|\vec{k}| \leq \Lambda / s$ for the case of critical phenomena and $|\vec{k}|<\epsilon H a$ for stochastic inflation.

Here,

$$
\begin{gathered}
\tilde{m}^{2}(t, s)=\tilde{m}^{2}(t)+\delta \tilde{m}^{2}(t, s)_{1}+\delta \tilde{m}^{2}(t, s)_{2 a}+\delta \tilde{m}^{2}(t, s)_{2 b} \\
\tilde{\lambda}(t, s,\{\vec{k}\})=\tilde{\lambda}(t)+\delta \tilde{\lambda}(t, s,\{\vec{k}\})_{1}
\end{gathered}
$$

The subscripts under $\delta \tilde{m}^{2}$ and $\delta \tilde{\lambda}$ denote the order of correction to the mass and the coupling constants arising from averaging over $\phi_{>}$fields. They are given by:

$$
\begin{gathered}
\delta \tilde{m}^{2}(t, s)_{2 a}=\left(-\frac{1}{4}\right)[i \delta(0)]^{2}[\tilde{\lambda}(t) / a(t)]^{2}(2 \pi)^{-3} \int d^{3} \vec{q}_{1}\left[\vec{q}_{1}^{2}+\tilde{m}^{2}(t)-i \epsilon\right]^{-2} \\
(2 \pi)^{-3} \int d^{3} \vec{q}_{2}\left[\vec{q}_{2}^{2}+\tilde{m}^{2}(t)-i \epsilon\right]^{-1} \\
\delta \tilde{m}^{2}(t, s)_{2 b}=\left(-\frac{1}{6}\right)[i \delta(0)]^{2}[\tilde{\lambda}(t) / a(t)]^{2}(2 \pi)^{-3} \int d^{3} \vec{q}_{1}(2 \pi)^{-3} \int d^{3} \vec{q}_{2}\left[\vec{q}_{1}^{2}+\tilde{m}^{2}(t)-i \epsilon\right]^{-1} \\
{\left[\vec{q}_{2}^{2}+\tilde{m}^{2}(t)-i \epsilon\right]^{-1}\left[\left(\vec{k}-\vec{q}_{1}-\vec{q}_{2}\right)^{2}+\tilde{m}^{2}(t)-i \epsilon\right]^{-1}} \\
\delta \tilde{\lambda}(t, s,\{\vec{k}\})=[i \delta(0)] \frac{3}{2} a(t)^{-1} \tilde{\lambda}^{2}(t)(2 \pi)^{-3} \int d^{3} \vec{q}\left[\vec{q}^{2}+\tilde{m}^{2}(t)-i \epsilon\right]^{-1} \\
{\left[\left(\vec{k}_{1}+\vec{k}_{2}-\vec{q}\right)^{2}+\tilde{m}^{2}(t)+i \epsilon\right]^{-1}} \\
\nu_{6}(t, s,\{\vec{k}\})=-10 \tilde{\lambda}^{2}(t)\left[\left(\vec{k}_{1}+\vec{k}_{2}+\vec{k}_{3}\right)^{2} \tilde{m}^{2}(t)-i \epsilon\right]^{-1}
\end{gathered}
$$




\section{BACKREACTION IN THE INFLATIONARY UNIVERSE: RENORMALIZATION GROUP EQUATIONS AND THE RUNNING OF COUPLING CONSTANTS}

We have so far discussed the separation and averaging processes leading to a coarse-grained effective action

which contains the averaged effect of the environment on the system. Now we will introduce a rescaling of the $\mathrm{k}$-space (truncated in critical phenomena, enlarged in stochastic inflation) to bring it back to the original size (but with less content in $\mathrm{CP}$, more in SI). By requiring that this transformed Lagrangian has the same form as the one we started out with we obtain a set of renormalization group equations for the field parameters. At this point our earlier observation on the equivalence of scaling and inflation for the field and spacetime in question becomes relevant. That is, rescaling in a static picture serves the function of inflation in a dynamic picture. Our derivation here of the differential renormalization group equations is similar to the Wilson-Fisher [29] or the Wegner-Houghton methods [40].

After introducing a rescaling of the k-space (spatial dimension $\mathrm{D}=3$ ),

$$
\vec{k}^{\prime}=s \vec{k}, \quad \phi^{\prime}\left(\vec{k}^{\prime}, t\right)=s^{-\frac{D+2}{2}} \phi_{<}(\vec{k}, t)
$$

the effective action (IV.24) becomes

$$
\begin{gathered}
S_{e f f}\left[\phi^{\prime}\right]=\int d t a(t) \int d^{3} \vec{k}^{\prime}\left(-\frac{1}{2}\right) \phi^{\prime}\left(\vec{k}^{\prime}, t\right)\left[\vec{k}^{\prime 2}+s^{2} \tilde{m}^{2}(t, s)\right] \phi^{\prime}\left(\vec{k}^{\prime}, t\right) \\
+\int d t a(t) \int d \vec{k}_{1}^{\prime} \cdots d \vec{k}_{4}^{\prime}\left(-\frac{1}{4 !}\right) s^{4-D} \tilde{\lambda}\left(t, s,\left\{\vec{k}^{\prime}\right\}\right) \phi^{\prime}\left(\vec{k}_{1}^{\prime}, t\right) \cdots \phi^{\prime}\left(\vec{k}_{4}^{\prime}, t\right) \delta\left(\vec{k}_{1}^{\prime}+\vec{k}_{2}^{\prime}+\vec{k}_{3}^{\prime}+\vec{k}_{4}^{\prime}\right) \\
+\int d t a(t) \int d \vec{k}_{1}^{\prime} \cdots d \vec{k}_{6}^{\prime}\left(-\frac{1}{6 !}\right) s^{6-2 D} \nu_{6}\left(t, s,\left\{\vec{k}^{\prime}\right\}\right) \phi^{\prime}\left(\vec{k}_{1}^{\prime}, t\right) \cdots \phi^{\prime}\left(\vec{k}_{6}^{\prime}, t\right) \delta\left(\vec{k}_{1}^{\prime}+\cdots+\vec{k}_{6}^{\prime}\right)
\end{gathered}
$$

Note that the $k^{\prime}$-integrations now resume the full range as $\mathrm{k}$ in (IV.7) or (IV.8). In terms of the rescaled variables $\phi^{\prime}, \mathrm{S}_{\mathrm{eff}}$ will have the same form up to a certain order as the original $\mathrm{S}_{\text {eff }}$ in terms of $\phi$ provided that we identify

$$
\begin{gathered}
\tilde{m}^{\prime 2}(t, s)=s^{2} \tilde{m}^{2}(t, s) \\
\tilde{\lambda}^{\prime}(t, s)=s^{4-D} \tilde{\lambda}(t, s)
\end{gathered}
$$

The effective mass and coupling constants are given in (IV.25. IV.29).

We can now proceed to tackle the coarse-grained integrals therein. At this point one needs to stipulate the system and bath separation such as the Cases A and B given as examples in Section 3. We will work out the details for critical phenomena (Case A) here. The case for stochastic inflation (Case B) can be obtained via the simple relation between Case A and B also mentioned in Section 3. For small changes in $s \simeq 1+d \sigma$, one can derive a set of differential renormalization group equations for the mass and the coupling constant as follows:

$$
\begin{array}{r}
d x / d \sigma=2 x-\frac{1}{2} y /(1+x) \\
d y / d \sigma=\epsilon y+\frac{3}{2} y^{2} /(1+x)^{2}
\end{array}
$$

where

$$
\begin{array}{r}
x \equiv \tilde{m}^{2}(t, \sigma) \Lambda^{-2} \\
y \equiv \tilde{\lambda}(t, \sigma) \Lambda^{-\epsilon} \Omega a^{-1}(t)
\end{array}
$$


Here, $\epsilon=4-D$, and $\Omega$ is the solid angle integration in D-dimension.

As expected, these equations have the same form as in the Ginzburg-Landau- Wilson model [29] 30 [41] - They govern the flow of the field parameters $\left(\tilde{m}^{2}, \tilde{\lambda}\right)$ as the scaling changes. There exist certain points in this parameter space known as the fixed points, where further application of the RG transformation leads to an invariant result. The fixed points are thus the steady-state solutions to the RG equations $d x / d \sigma=0$ and $d y / d \sigma=0$. For the $\phi^{4}$ theory, it is well-known that there is a trivial fixed point at

$$
x_{f}^{0}=0, \quad y_{f}^{0}=0
$$

and a nontrivial fixed point at

$$
x_{f}^{*}=-\epsilon / 6, \quad y_{f}^{*}=-2 \epsilon / 3
$$

Near the trivial fixed point the solution to (V.4) is

$$
\left(\begin{array}{l}
x \\
y
\end{array}\right)=A\left(\begin{array}{l}
1 \\
0
\end{array}\right) e^{2 \sigma}+B\left(\begin{array}{c}
1 \\
2(2-\epsilon)
\end{array}\right) e^{\epsilon \sigma}
$$

The critical point of interest to us is the nontrivial fixed point. We want to find out how in its neighborhood the field parameters flow towards this fixed point. By setting $x=x_{f}^{*}+\Delta x$ and $y=y_{f}^{*}+\Delta y$ we get

$$
d(\Delta x) / d \sigma=\left(2-\frac{1}{3} \epsilon\right) \Delta x-\frac{1}{2}\left(1+\frac{1}{6} \epsilon\right) \Delta y
$$

and

$$
d(\Delta y) / d \sigma=-\epsilon \Delta y
$$

with the solution

$$
\left(\begin{array}{c}
\Delta x \\
\Delta y
\end{array}\right)=A\left(\begin{array}{l}
1 \\
0
\end{array}\right) e^{(2-\epsilon / 3) \sigma}+B\left(\begin{array}{c}
1 \\
4(1+\epsilon / 6)
\end{array}\right) e^{-\epsilon \sigma}
$$

\section{A. Cosmological Consequences}

As we explained earlier, in eternal inflation the renormalization group transformation parameterized by $s$ is equivalent to time evolution parametrized by $t$. Thus under such conditions we can treat the coupling constants like $\lambda(t, s)$ as depending on either $s$ (flow) or $t$ (evolution). The fixed point corresponds to the extreme infrared limit which in the case of eternal inflation lies at infinitely late time $t_{f}$. In practice it corresponds to the time the inflation regime ends $t_{1} \simeq t_{f}$ before reheating sets in. Thus in (V.10) $\Delta y$ is a measure of how $y$ changes from $t$ to $t_{1}$. Explicitly, with $\epsilon=1$ and $\sigma=\int H d t$, the $O(\epsilon)$ expansion result gives

$$
y(t)=y\left(t_{f}\right)+\frac{14 B}{3} e^{-H\left(t_{f}-t\right)} .
$$

At the fixed point $t_{f}, y\left(t_{f}\right)=-\frac{2}{3} \epsilon$. Recall that $y=\tilde{\lambda(t)} \Lambda^{-\epsilon} \Omega a^{-1}(t)$ and $\tilde{\lambda}(t)=\lambda(t) a^{2}(t)$,this yields

$$
\lambda(t)=-\frac{2}{3} e^{-H\left(t-t_{f}\right)}\left[1-7 B e^{H\left(t-t_{f}\right)}\right] .
$$

Comparing $\lambda(t)$ at two times $t>t_{0}$,

$$
\frac{\lambda(t)}{\lambda\left(t_{0}\right)} \simeq e^{-H\left(t-t_{0}\right)}\left[1-7 B\left(e^{H\left(t-t_{f}\right)}-e^{H\left(t_{0}-t_{f}\right)}\right)\right] .
$$

Assuming $t_{f}>t, t_{0}$ (which is clearly satisfied for realistic inflation $t_{f}>t_{0} e^{68}$ ) the second term in the square bracket can be neglected. We get, 


$$
\lambda(t) / \lambda\left(t_{0}\right) \simeq e^{-H\left(t-t_{0}\right)}
$$

Thus the coupling constant $\lambda$ of the effective field theory (in a system which is depleting in content) actually decreases with time. 5 This is a new effect which could have important consequence for the galaxy formation problem.

As we know from standard calculations [43] the density contrast $\delta \rho / \rho$ depends strongly on $\lambda$. For GUT processes one needs to assume an unnaturally weak $\lambda \approx 10^{-12}$ in order that $\delta \rho / \rho \approx 10^{-4}$ at the time the fluctuations reenter the (RW) horizon. What (V.14) implies is that the strength of $\lambda$ at time $t_{G}^{*}$ when the fluctuation mode corresponding to galaxy scale left the de Sitter horizon is much weaker than $\lambda$ at $t_{H}^{*}$, the time the Hubble size left the de Sitter horizon. The running of the coupling constants seems to provide a way to reduce their strength. The reason behind this phenomena is, as we recall, due to the backreaction of the environment of high $\mathrm{k}$ modes on the system of lower modes, which in this particular choice is diminishing in time.

In contrast, for stochastic inflation where the system (consisting of modes with physical wavelength greater than the de Sitter horizon) increases in content, the coupling constants will, from this analysis, actually increase exponentially with time during the inflationary regime (era from $t_{0}$ to $t_{1}$ ). Because a formal analogy exists between this case (Case B) of stochastic inflation (SI) and critical phenomena (CP, Case A) one can indeed just read off the result from the results we obtained earlier. The transcription is simply i) Changing $\Lambda$ in $\mathrm{CP}$ to $\epsilon H$ in SI. Recall that $\Lambda$ refers to the ultraviolet cutoff in CP, whereas $H^{-1}$ is the horizon size. The ultraviolet cutoff for SI is assumed to be $\infty$. ii) Changing $s$ in CP to $s^{-1}$ in SI. Recall that the coarse-graining parameter $s$ plays the role of the scale function $a(t)$. Thus $s^{-1}$ in CP is now acting like $a(t)$ in SI [see Eq. (IV.8)]. Note that $s>1$ in CP but $s<1$ in SI. Note also that transformation (ii) is equivalent to a time reversal, i.e., changing $\Delta t \rightarrow-\Delta t$. The way $\lambda$ runs in stochastic inflation is given by Eq. (V.12), with $-t$ replacing $t$ there. The ensuing discussion of the implications on galaxy formation is similar, except that, of course, one reaches the opposite conclusion, i.e., the strengthening of $\lambda$ in time increases the density contrast as each mode reenters the RW horizon.

The opposite results arising from these two cases should not be viewed as unsettling. They arise from very different stipulations of the system (CP decreasing in time, SI increasing). The cosmological consequences will depend on whether and how $\lambda$ runs, but the theoretical issues this investigation brings out should be valid. What is shown here is that if one takes into account the interaction between the environment and the system, the running of coupling parameters in the theory as observed in the system is an unavoidable consequence. How they run (with time or scale or energy) will depend sensitively on how the system is selected with respect to the environment, and, of course, the nature of the interaction (gauge fields would presumably run differently from $\lambda \phi^{4}$, as their $U V$ and $I R$ behavior differ).

On this point, let us reexamine the physical criteria for the choice of the system versus the environment in our examples. The cutoff is determined by the highest $k$ mode which left the de Sitter horizon, which in turn depends on the duration of inflation. At the start of inflation of course there is no knowledge when inflation will end and the modes do not know which ones among them are to be counted in (the system) and which are to be out (the environment). A more straightforward division would be to have a fixed cutoff at the beginning of inflation, e.g., those with $\mathrm{k}$ greater than the galaxy size $\left(k_{G}\right)$ constitute the environment and those smaller than $k_{G}$ but greater than $H^{-1}$ be the system. This would give rise to running, but presumably at a different rate. Indeed, if one is interested in the behavior of a particular mode, say, that of the galaxy scale $k_{G}$, one can regard this one mode as the system and the rest as the environment. At all times this one mode is interacting with the rest of the whole spectrum, some of these leave the DS horizon earlier, some later. The behavior of this particular mode determines at a critical time $t_{G}^{*}$ (when it leaves the DS horizon) its own amplitude when it reenters the RW horizon, but it is influenced by all the lower and higher modes at earlier times. The effect of coarse-graining for this choice of the system (single mode) is similar to the minisuperspace problem in quantum cosmology. It is expected that the coupling constants will not run in these circumstances. Even in more general cases it may also be that the changing $\lambda$ as experienced by

\footnotetext{
${ }^{5}$ Of course, even though the running coupling goes like $\lambda(t) / \lambda\left(t_{0}\right) \simeq e^{-H\left(t-t_{0}\right)}$, a loop expansion of a quantity such as the density-density correlation function will show that in the same limit each loop appears with a factor of $e^{H\left(t-t_{0}\right)}$. The self-consistent cancellation of these two factors is associated with a crossover phenomenon and in principle could be accounted for by using an H-dependent (environmental-friendly) RG 42]. We thank C. R. Stephens for this remark.
} 
modes in the system may not exert any effect on galaxy formation, because at the moment any kth mode departs the de Sitter horizon it will have the same amplitude no matter what history of interaction it has experienced, and it is this amplitude which determines the density contrast for galaxy formation in the RW era. This point, however, raises an interesting theoretical issue.

\section{B. Theoretical Implications}

We see that different choices of the integration range in the calculation of the CGEA give rise to very different behavior of the coupling constants. In critical phenomena we stipulated the system to be in the same way as the real space renormalization group treatment of critical phenomena, i.e., that the ultraviolet cutoff is fixed at the start of inflation- this corresponds to the inverse of the lattice size, which is a fixed value for condensed matter considerations. In each iteration the UV cutoff is redshifted, making the effective range of frequency smaller at successive periods. In critical phenomena scaling is an artificial procedure to facilitate the approach to the infrared limit. However, in inflation every different time interval corresponds to a realistic physical situation. If one adopts the coarse-graining scheme similar to critical phenomena, one would get a diminishing range for the system as the universe expands. This is the cause of the changing coupling constant, and is also the source of a serious problem. Imagine that if one turns the problem around by demanding that the physics observed in each of the subsequent moments should be identical, specifically, if it possesses the full range from the Planck scale to infinity (k from 0 to inverse Planck length)- this is certainly what one usually assumes, otherwise the physical range we observe in the post-inflationary era such as in today's universe would only consists of the low k modes-if one makes this reasonable assertion, then we would not have the running coupling constants problem. This is equivalent to assuming that the physical wavemodes $p=k / a$ rather than the intrinsic wavemodes $\mathrm{k}$ has the integration range between zero and the inverse Planck length. However, making such an adjustment in order to make the range identical at each subsequent moment would require a mechanism to 'replenish' the high frequency modes between the redshifted UV limit and the Planck length, which would open up another serious problem. Notice that this problem is not particular to inflation nor curved spacetime. It is already there for any theory in a dynamical setting, e.g., in cosmology. It only becomes more serious in inflation because the redshifting is exponential. Similar questions would arise in black holes when one tries to compare what is observed between different observers from the event horizon to asymptotic infinity. The relation between black hole and inflation can be easily understood by viewing them in terms of exponential scaling, as in the dynamical finite size effects [28.

One can analyze this problem in the context of the renormalization group theory by using an explicit cutoff (defined at one instant as the Planck scale). By demanding that this cutoff be the same at all instants and at all energies, one would presumably not get any flow. This would in turn demand some theory on the physical consequence of transPlanckian frequencies 6

\section{PART THREE. Backreaction and Noise from CTP- CGEA}

\section{THE CLOSED-TIME-PATH COARSE- GRAINED EFFECTIVE ACTION}

As we introduced the CGEA in the 'in-out' version [4] we remarked that for a correct treatment of backreaction problems, the 'in-in' or closed-time-path CTP version of CGEA is the right way to proceed, as it gives a real and causal equation of motion for the effective dynamics of the open system. The CTP CGEA was first introduced by $\mathrm{Hu}$ and Sinha 35] to analyze the validity of the minisuperspace approximation in quantum cosmology. The influence functional method [45] for interacting quantum fields (based on quantum Brownian

\footnotetext{
${ }^{6}$ This issue raised originally in the context of inflationary cosmology in has a parallel in black holes, which was pursued independently by Jacobson, Unruh and others. For a discussion of trans-Planckian modes problems and explorations for its implication for quantum gravity, see, e.g., 44 .
} 
motion [46]) was introduced by $\mathrm{Hu}, \mathrm{Paz}$ and Zhang in [6, 47,8] and Calzetta and $\mathrm{Hu}$ [7], and applied to a range of problems in semiclassical gravity and cosmology such as decoherence, structure formation, entropy generation and reheating [32,33,48, 49. Lombardo and Mazzitelli [50] computed perturbatively the CTP CGEA for a self-interacting theory in a Robertson-Walker spacetime to discuss the quantum to classical transition of the low modes of the field, and to put on firmer grounds the quantum theory of structure formation in inflationary models. Greiner and Müller [51] also used the CTP CGEA to analyze the classical limit of the soft modes of a quantum field when the hard modes are in thermal equilibrium. Dalvit and Mazzitelli [52] showed that it is possible to write an exact equation for the dependence of the CGEA on the scale that separates the system and the environment. This is expected to be useful for a nonperturbative calculation of the CGEA, and to discuss the appearance of noise in the renormalization group equations.

In this part we will review the above mentioned works. We will start with the necessary definitions and the perturbative evaluation of the CTP CGEA for a $\lambda \phi^{4}$ scalar field in a Friedmann-Robertson-Walker (FRW) background spacetime. We will describe how to use the CTP CGEA to analyze the validity of the minisuperspace approximation in quantum cosmology and the issue of quantum to classical transition of fluctuations which give rise to structure formation in inflationary models. We will finally describe attempts to compute the CTP CGEA using non perturbative approximations.

\section{A. Perturbative evaluation of the CTP CGEA}

We consider again the scalar field action given by Eq.(III.1). In a flat Robertson-Walker spacetime with metric $d s^{2}=d t^{2}-a^{2}(t) d \vec{x}^{2}=a^{2}(\eta)\left[d \eta^{2}-d \vec{x}^{2}\right]$, the action can be written as

$$
S(a, \chi)=\int d^{4} x\left[\frac{1}{2} \eta^{\mu \nu} \partial_{\mu} \chi \partial_{\nu} \chi-\frac{1}{2} m^{2} a^{2} \chi^{2}-\frac{1}{2}\left(\xi-\frac{1}{6}\right) R a^{2} \chi^{2}-\frac{\lambda}{4 !} \chi^{4}\right]
$$

where $\chi=a \phi$. From now on, $d^{4} x$ denotes $d^{3} x d \eta$. To see the flat space results simply set $a=1$ and $R=0$. Let us make a system-environment field splitting

$$
\chi(x)=\chi_{<}(x)+\chi_{>}(x),
$$

where we define the system by

$$
\chi_{<}(\vec{x}, t)=\int_{|\vec{k}|<\Lambda_{c}} \frac{d^{3} \vec{k}}{(2 \pi)^{3}} \phi(\vec{k}, \eta) \exp i \vec{k} \cdot \vec{x}
$$

and the environment by

$$
\chi>(\vec{x}, \eta)=\int_{|\vec{k}|>\Lambda_{c}} \frac{d^{3} \vec{k}}{(2 \pi)^{3}} \phi(\vec{k}, \eta) \exp i \vec{k} \cdot \vec{x} .
$$

The system-field contains the modes with wavelengths longer than the critical value $\Lambda_{c}^{-1}$, while the bath or environment-field contains wavelengths shorter than $\Lambda_{c}^{-1} . \Lambda_{c}$ corresponds to $\frac{\Lambda}{s}$ of previous sections.

After the splitting, the total action (VI.1) can be written as

$$
S[a, \chi]=S_{0}\left[\chi_{<}\right]+S_{0}\left[\chi_{>}\right]+S_{\text {int }}\left[a, \chi_{<}, \chi_{>}\right],
$$

where $S_{0}$ denotes the kinetic term and the interaction part is given by

$$
\begin{aligned}
& S_{\text {int }}\left[a, \chi_{<}, \chi_{>}\right]=-\int d^{4} x\left\{\frac{M^{2}}{2} \chi_{<}^{2}+\frac{\lambda}{4 !} \chi_{<}^{4}+\frac{M^{2}}{2} \chi_{>}^{2}+\frac{\lambda}{4 !} \chi_{>}^{4}+\right. \\
& \left.\frac{\lambda}{4} \chi_{<}^{2}(x) \chi_{>}^{2}+\frac{\lambda}{6} \chi_{<}^{3} \chi_{>}+\frac{\lambda}{6} \chi_{<} \chi_{>}^{3}\right\} .
\end{aligned}
$$

with $M^{2}=m^{2} a^{2}+\left(\xi-\frac{1}{6}\right) R a^{2}$.

We are interested in the influence of the environment on the evolution of the system. Therefore the CTP CGEA $S_{\Lambda}\left[a, \chi_{<}, a^{\prime}, \chi_{<}^{\prime}\right]$ is the object of relevance. It is defined by 


$$
\begin{aligned}
& \exp i S_{\Lambda_{c}}\left[a, \chi_{<}, a^{\prime}, \chi_{<}^{\prime}\right]=\exp i\left(S_{0}\left[\chi_{<}\right]-S_{0}\left[\chi_{<}^{\prime}\right]\right) \int d \chi_{>f} \int^{\chi_{>f}} \mathcal{D} \chi_{>} \int^{\chi_{>f}} \mathcal{D} \chi_{>}^{\prime} \\
& \times \exp i\left\{S_{0}\left[\chi_{>}\right]+S_{i n t}\left[a, \chi_{<}, \chi_{>}\right]-S_{0}\left[\chi_{>}^{\prime}\right]-S_{i n t}\left[a^{\prime}, \chi_{<}^{\prime}, \chi_{>}^{\prime}\right]\right\} .
\end{aligned}
$$

The integration is over all fields $\chi_{>}\left(\right.$and $\chi_{>}^{\prime}$ ) with positive (and negative) frequency modes in the remote past that coincide at the final time $\chi_{>}=\chi_{>}^{\prime}=\chi_{>f}$

We will calculate the CTP CGEA perturbatively in $\lambda$ and $M^{2}$, up to quadratic order in both quantities. After a simple calculation we obtain

$$
\begin{aligned}
& S_{\Lambda_{c}}\left[a, \chi_{<}, a^{\prime}, \chi_{<}^{\prime}\right]=S_{0}\left[\chi_{<}\right]-S_{0}\left[\chi_{<}^{\prime}\right]+\left\{\left\langle S_{i n t}\left[a, \chi_{<}, \chi_{>}\right]\right\rangle_{0}-\left\langle S_{i n t}\left[a^{\prime}, \chi_{<}^{\prime}, \chi_{>}^{\prime}\right]\right\rangle_{0}\right\} \\
& +\frac{i}{2}\left\{\left\langle S_{i n t}^{2}\left[a, \chi_{<}, \chi_{>}\right]\right\rangle_{0}-\left[\left\langle S_{i n t}\left[a, \chi_{<}, \chi_{>}\right]\right\rangle_{0}\right]^{2}\right\} \\
& -i\left\{\left\langle S_{i n t}\left[a, \chi_{<}, \chi_{>}\right] S_{i n t}\left[a^{\prime}, \chi_{<}^{\prime}, \chi_{>}^{\prime}\right]\right\rangle_{0}-\left\langle S_{\text {int }}\left[a, \chi_{<}, \chi_{>}\right]\right\rangle_{0}\left\langle S_{i n t}\left[a^{\prime}, \chi_{<}^{\prime}, \chi_{>}^{\prime}\right]\right\rangle_{0}\right\} \\
& +\frac{i}{2}\left\{\left\langle S_{\text {int }}^{2}\left[a^{\prime}, \chi_{<}^{\prime}, \chi_{>}^{\prime}\right]\right\rangle_{0}-\left[\left\langle S_{\text {int }}\left[a^{\prime} \chi_{<}^{\prime}, \chi_{>}^{\prime}\right]\right\rangle_{0}\right]^{2}\right\}
\end{aligned}
$$

where the quantum average of a functional of the fields is defined with respect to the free action $S_{0}$

$$
\left\langle B\left[\chi_{>}, \chi_{>}^{\prime}\right]\right\rangle_{0}=\int d \chi_{>f} \int^{\chi_{>f}} \mathcal{D} \chi_{>} \int^{\chi_{>f}} \mathcal{D} \chi_{>}^{\prime} \exp \left\{S_{0}\left[\chi_{>}\right]-S_{0}\left[\chi_{>}^{\prime}\right]\right\} B .
$$

Equation (VI.8) is the in-in version of the Dyson-Feynman series (IV.20).

We define the propagators of the environment field as

$$
\begin{gathered}
\left\langle\chi_{>}(x) \chi_{>}(y)\right\rangle_{0}=i G_{++}^{\Lambda_{c}}(x-y), \\
\left\langle\chi_{>}(x) \chi_{>}^{\prime}(y)\right\rangle_{0}=-i G_{+-}^{\Lambda_{c}}(x-y), \\
\left\langle\chi_{>}^{\prime}(x) \chi_{>}^{\prime}(y)\right\rangle_{0}=-i G_{--}^{\Lambda_{c}}(x-y) .
\end{gathered}
$$

These propagators are not the usual Feynman, positive-frequency Wightman, and Dyson propagators of the scalar field since, in this case, the momentum integration is restricted by the presence of the (infrared) cutoff $\Lambda_{c}$. The explicit expressions are

$$
\begin{gathered}
G_{++}^{\Lambda_{c}}(x-y)=\int_{|\vec{p}|>\Lambda_{c}} \frac{d^{4} p}{(2 \pi)^{4}} e^{i p(x-y)} \frac{1}{p^{2}+i \epsilon}, \\
G_{+-}^{\Lambda_{c}}(x-y)=-\int_{|\vec{p}|>\Lambda_{c}} \frac{d^{4} p}{(2 \pi)^{4}} e^{i p(x-y)} 2 \pi i \delta\left(p^{2}\right) \Theta\left(p^{0}\right), \\
G_{--}^{\Lambda_{c}}(x-y)=\int_{|\vec{p}|>\Lambda_{c}} \frac{d^{4} p}{(2 \pi)^{4}} e^{i p(x-y)} \frac{1}{p^{2}-i \epsilon} .
\end{gathered}
$$

As an example, we show the expression for the propagator $G_{++}^{\Lambda_{c}}$. The usual Feynman propagator is

$$
G_{++}(x)=\frac{i}{8 \pi^{2}} \frac{1}{\sigma}-\frac{1}{8 \pi} \delta(\sigma),
$$

while

$$
\begin{aligned}
& G_{++}^{\Lambda_{c}}(x)=\frac{i}{8 \pi^{2}}\left[\frac{\cos \left[\Lambda_{c}\left(r-x^{0}\right)\right]}{r\left(r-x^{0}\right)}+\frac{\cos \left[\Lambda_{c}\left(r+x^{0}\right)\right]}{r\left(r+x^{0}\right)}\right]-\frac{1}{8 \pi^{2}}\left[\frac{\sin \left[\Lambda_{c}\left(r-x^{0}\right)\right]}{r\left(r-x^{0}\right)}-\frac{\sin \left[\Lambda_{c}\left(r+x^{0}\right)\right]}{r\left(r+x^{0}\right)}\right] \\
& \equiv G_{++}(x)-G_{++}^{|\vec{p}|<\Lambda_{c}}(x),
\end{aligned}
$$


where $\sigma=\frac{1}{2} x^{2}$.

The CTP CGEA can be computed from Eqs. (VI.8)-(VI.12) using standard techniques. After some algebra we find

$$
\begin{aligned}
& S_{\Lambda_{c}}\left[a, \chi_{<}, a^{\prime}, \chi_{<}^{\prime}\right]=S_{0}\left(\chi_{<}\right)-S_{0}\left(\chi_{<}^{\prime}\right)-\frac{1}{2} \int d^{4} x\left(M^{2}(x) \chi_{<}^{2}-M^{\prime 2} \chi_{<}^{\prime 2}\right) \\
& -\lambda \int d^{4} x\left[\frac{1}{24}\left(\chi_{<}^{4}(x)-\chi_{<}^{\prime 4}(x)\right)+\frac{1}{2} i G_{++}^{\Lambda_{c}}(0)\left(\tilde{M}^{2}(x)-\tilde{M}^{\prime 2}(x)\right)\right] \\
& +\int d^{4} x \int d^{4} y\left[-\frac{\lambda^{2}}{72} \chi_{<}^{3}(x) G_{++}^{\Lambda_{c}}(x-y) \chi_{<}^{3}(y)-\frac{\lambda^{2}}{36} \chi_{<}^{3}(x) G_{+-}^{\Lambda_{c}}(x-y) \chi_{<}^{\prime 3}(y)\right. \\
& +\frac{\lambda^{2}}{72} \chi_{<}^{\prime 3}(x) G_{--}^{\Lambda_{c}}(x-y) \chi_{<}^{\prime 3}(y)-\frac{1}{4} \tilde{M}^{2}(x) i G_{++}^{\Lambda_{c} 2}(x-y) \tilde{M}^{2}(y) \\
& +\frac{1}{2} \tilde{M}^{2}(x) i G_{+-}^{\Lambda_{c} 2}(x-y) \tilde{M}^{\prime 2}(y)-\frac{1}{4} \tilde{M}^{\prime 2}(x) i G_{--}^{\Lambda_{c} 2}(x-y) \tilde{M}^{\prime 2}(y) \\
& +\frac{\lambda^{2}}{18} \chi_{<}(x) G_{++}^{\Lambda_{c} 3}(x-y) \chi_{<}(y)+\frac{\lambda^{2}}{9} \chi_{<}(x) G_{+-}^{\Lambda_{c} 3}(x-y) \chi_{<}^{\prime}(y) \\
& \left.-\frac{\lambda^{2}}{18} \chi_{<}^{\prime}(x) G_{--}^{\Lambda_{c} 3}(x-y) \chi_{<}^{\prime}(y)\right],
\end{aligned}
$$

where we introduced the notation $\tilde{M}^{2}=M^{2}+\frac{\lambda}{2} \chi_{<}^{2}$.

Defining

$$
\begin{gathered}
P_{ \pm}=\frac{1}{2}\left(\chi_{<}^{4} \pm \chi_{<}^{\prime 4}\right) \quad ; \quad R_{ \pm}=\frac{1}{2}\left(\chi_{<}^{3} \pm \chi_{<}^{\prime 3}\right) \\
\lambda Q_{ \pm}=\frac{1}{2}\left(\tilde{M}^{2} \pm \tilde{M}^{\prime 2}\right) \quad ; \quad \chi_{ \pm}=\frac{1}{2}\left(\chi_{<} \pm \chi_{<}^{\prime}\right),
\end{gathered}
$$

and using simple identities for the propagators, the real and imaginary parts of the CTP CGEA can be written as

$$
\begin{aligned}
& R e S_{\Lambda_{c}}=S_{0}\left(\chi_{<}\right)-S_{0}\left(\chi_{<}^{\prime}\right)-\lambda \int d^{4} x\left\{\frac{1}{12} P_{-}(x)+\frac{i}{2} G_{++}^{\Lambda_{c}}(0) Q_{-}(x)\right\} \\
& +\lambda^{2} \int d^{4} x \int d^{4} y \Theta\left(y^{0}-x^{0}\right)\left\{-\frac{1}{18} R_{+}(x) \operatorname{Re} G_{++}^{\Lambda_{c}}(x-y) R_{-}(y)+\right. \\
& \left.\frac{1}{4} Q_{+}(x) \operatorname{Im} G_{++}^{\Lambda_{c} 2}(x-y) Q_{-}(y)+\frac{1}{3} \chi_{+}(x) \operatorname{Re} G_{++}^{\Lambda_{c} 3}(x-y) \chi_{-}(y)\right\}, \\
& I m S_{\Lambda_{c}}=\lambda^{2} \int d^{4} x \int d^{4} y\left\{-\frac{1}{18} R_{-}(x) \operatorname{Im} G_{++}^{\Lambda_{c}}(x-y) R_{-}(y)\right. \\
& \left.-\frac{1}{4} Q_{-}(x) \operatorname{Re} G_{++}^{\Lambda_{c} 2}(x-y) Q_{-}(y)+\frac{1}{3} \chi_{-}(x) \operatorname{Im} G_{++}^{\Lambda_{c} 3}(x-y) \chi_{-}(y)\right\} .
\end{aligned}
$$

The real part of the CTP CGEA in Eq. VI.19) contains divergences and must be renormalized. As the propagators in Eqs. (VI.10)-(VI.12) differ from the usual ones only by the presence of the infrared cutoff, the ultraviolet divergences coincide with those of the usual $\lambda \chi^{4}$-theory. The effective action can therefore be renormalized using the standard procedure.

Consider the square of the Feynman propagator. Using dimensional regularization we find

$$
G_{++}^{\Lambda_{c} 2}(x)=G_{++}^{2}(x)+G_{++}^{\left(|\vec{p}|<\Lambda_{c}\right) 2}(x)-2 G_{++}(x) G_{++}^{\left(|\vec{p}|<\Lambda_{c}\right)}(x),
$$

where

$$
\begin{gathered}
G_{++}^{2}(x)=\frac{i}{16 \pi^{2}}\left[\frac{1}{n-4}+\psi(1)-4 \pi\right] \delta^{4}(x)+i \Sigma(x)-\eta(x)-\log \left[4 \pi \mu^{2}\right], \\
\Sigma(x)=\frac{1}{(2 \pi)^{4}} \int d^{4} p e^{i p x} \log \left|p^{2}\right|
\end{gathered}
$$




$$
\eta(x)=\frac{\pi}{(2 \pi)^{4}} \int d^{4} p e^{i p x} \Theta\left(p^{2}\right) .
$$

Note that the divergence is the usual one, i.e., proportional to $\frac{1}{n-4} \delta^{4}(x-y)$ and independent of $\Lambda_{c}$. Consequently, the term $\operatorname{Im} G_{++}^{\Lambda 2}(x-y) Q_{+}(x) Q_{-}(y)$ in Eq. (VI.19) is also divergent and renormalizes the coupling constant $\lambda$ and the constants that appear in the gravitational action (as usual, in order to renormalize the theory of a quantum field in a curved space, it is necessary to include in the gravitational action the Einstein Hilbert term, a cosmological constant, and terms quadratic in the curvature tensor). The other divergences can be treated in a similar way. One can also check that the imaginary part of the effective action does not contain divergences.7

As we will show with the help of QBM models, the (nonlocal) real and imaginary parts of $S_{\Lambda_{c}}\left[a, \chi_{<}, a^{\prime}, \chi_{<}^{\prime}\right]$ can be associated with the dissipation and noise respectively, and can be related by an integral equation known as the fluctuation-dissipation relation.

Similar perturbative results for the CTP CGEA have been obtained by Greiner and Müller in Ref. [51]. In order to derive effective field equations for the soft modes of the scalar field, they computed the CTP CGEA assuming that the modes in the environment are at thermal equilibrium at a temperature $T$ such that $\Lambda_{c} \ll T$. Their result for the effective action is essentially given by expressions (VI.19) and (VI.20), for the particular case $a(t)=1$ and replacing the vacuum propagators by thermal propagators in order to take into account the state of the environment.

In Section VII.B we will see that the field equations derived from the CTP CGEA are real and causal.

\section{B. Applications: Dynamics of System Fields/Modes with Backreaction of Environment Fields/Modes}

The coarse-grained effective action is a very useful method to treat coarse-graining and backreaction problems. Examples include stochastic inflation [6,32,50] and reheating in inflationary cosmology [33, 53], effect of hard thermal loops in QCD plasma, and the effect of individual atoms on a BEC condensate. The first instances the CTP effective action was applied to were for backreaction of quantum fluctuations and particle creation on the background spacetime [1, 39] or for interacting quantum fields [2,54]. The CTP CGEA was first introduced [35] to address the validity of the so-called minisuperspace approximation. Although it was introduced in the context of quantum cosmology, the method has a wide range of applications. The relevant issues are the backreaction effect of the inhomogeneous modes in an interacting quantum field on the homogeneous mode; and the validity of quantizing a truncated theory: does it preserve the salient features of a fully quantized theory? These issues underlie many problems in physics, especially when we view effective theories as playing a more fundamental role in the description of Nature [55].

\section{MASTER AND LANGEVIN EQUATIONS IN QUANTUM FIELD THEORY}

We now show how to use the open system concepts and techniques to give a first principles derivation of the evolution equation for the reduced density matrix that describes the system under the influence of the environment. We will use the influence functional method to extract the noise and dissipation kernels. The open system framework is best illustrated by the quantum mechanical problem of Brownian motion (QBM). Feynman and Vernon 445] first treated this problem with the influence functional method. Using this method, the master equation was derived by Caldeira and Leggett [46] for Markovian processes (Ohmic environment at high temperature), and by $\mathrm{Hu} \mathrm{Paz}$ and Zhang [56] for a general environment including nonMarkovian processes. (See also [57] and [58]). As we will soon find out, the influence functional method is intimately related to the CTP coarse-grained effective action [59.7].

\footnotetext{
${ }^{7}$ Of course, a successful ultraviolet renormalization does not guarantee that an approximation scheme such as RGimproved perturbation theory will be well behaved. A good example is in Section V, where loops depend on a factor $e^{H\left(t-t_{0}\right)}$ which would invalidate perturbation theory. Further 'infrared' $H$-dependent (environmentally friendly) renormalization of $\lambda$ is needed.
} 
$\mathrm{Hu}, \mathrm{Paz}$ and Zhang [47,6] first generalized the quantum mechanical problem of Brownian motion to quantum fields, taking the system and environment as two independent fields. Lombardo and Mazzitelli 50] treated the same problem, taking as system and environment the low and high frequency modes of a single, self-interacting scalar field. We will follow their treatment here.

We begin with a brief review of the problem of quantum Brownian motion. Denote by $x$ the coordinate of the Brownian particle, by $\Omega$ its frequency, and by $q_{i}$ the coordinates of the oscillators in the environment. The influence of the environment on the Brownian particle can be described by the reduced density matrix $\rho_{r}\left(x, x^{\prime}, t\right)$ that is obtained from the full density matrix by integrating out the environmental degrees of freedom $q_{i}$.

For a linear coupling $x q_{i}$, the master equation for the reduced density matrix $\rho_{r}\left(x, x^{\prime}, t\right)$ is of the form [60]

$$
\begin{aligned}
& i \hbar \partial_{t} \rho_{r}\left(x, x^{\prime}, t\right)=\left\langle x\left|\left[H, \rho_{r}\right]\right| x^{\prime}\right\rangle-i \gamma(t)\left(x-x^{\prime}\right)\left(\partial_{x}-\partial_{x^{\prime}}\right) \rho_{r}\left(x, x^{\prime}, t\right) \\
& +f(t)\left(x-x^{\prime}\right)\left(\partial_{x}+\partial_{x^{\prime}}\right) \rho_{r}\left(x, x^{\prime}, t\right)-i D(t)\left(x-x^{\prime}\right)^{2} \rho_{r}\left(x, x^{\prime}, t\right),
\end{aligned}
$$

where the coefficients $\gamma(t), D(t)$ and $f(t)$ depend on the properties of the environment (temperature $\beta^{-1}$ and spectral density $I(\omega)$ ). The first term on the RHS of Eq. (VII.1) gives the usual Liouvillian evolution. The second is a dissipative term with a time dependent dissipative coefficient $\gamma(t)$. The last two are diffusive terms. The one proportional to the anomalous diffusion coefficient $f(t)$ does not produce decoherence, i.e., the off-diagonal terms in the density matrix are not suppressed in time. To see this in a simple example, assume that $\rho_{r}=\rho_{0} e^{-B(t)\left(x-x^{\prime}\right)^{2}}$. Inserting this into Eq. (VII.1) it is easy to prove that the $f$-term produces an oscillating function $B(t)$ but no damping. (We refer the reader to Refs. 60, 61] for a more detailed justification). The last term with diffusion coefficient $D(t)$

$$
D(t)=\int_{0}^{t} d s \cos (\Omega s) \quad \int_{0}^{\infty} d \omega I(\omega) \operatorname{coth}\left(\frac{1}{2} \beta \hbar \omega\right) \cos (\omega s),
$$

gives the main contribution to decoherence. Indeed, an approximate solution of Eq. (VII.1) is [56,60]

$$
\rho_{r}\left[x, x^{\prime} ; t\right] \approx \rho_{r}\left[x, x^{\prime}, 0\right] \exp \left[-\left(x-x^{\prime}\right)^{2} \int_{0}^{t} D(s) d s\right],
$$

and we see that the off-diagonal terms of the density matrix are suppressed as long as $\int_{0}^{t} D(s) d s$ is large enough. For non-linear couplings like $x^{n} q_{i}^{m}$, one expects the master equation to contain terms of the form $i D^{(n, m)}(t)\left(x^{n}-x^{\prime n}\right)^{2} \rho_{r}$.

We now proceed to quantum field theory. The total density matrix (for the system and environment fields) is defined by

$$
\rho\left[\chi_{<,} \chi_{>}, \chi_{<}^{\prime}, \chi_{>}^{\prime}, \eta\right]=\left\langle\chi<\chi_{>}|\hat{\rho}| \chi_{<}^{\prime} \chi_{>}^{\prime}\right\rangle,
$$

where $\left|\chi_{<}\right\rangle$and $\left|\chi_{>}\right\rangle$are the eigenstates of the field operators $\hat{\chi}_{<}$and $\hat{\chi}>$, respectively. For simplicity, we will assume that the interaction is turned on at the initial time $\eta_{0}$ and that, at this time, the system and the environment are not correlated. (The physical consequences of such a choice is elaborated in [56]. More general initial conditions can be introduced by a stipulated preparation function [62].) As such, the total density matrix can be written as the product of the density matrix operator for the system and for the bath

$$
\hat{\rho}\left[\eta_{0}\right]=\hat{\rho}_{<}\left[\eta_{0}\right] \hat{\rho}_{>}\left[\eta_{0}\right] .
$$

We will further assume that the initial state of the environment is the vacuum.

The reduced density matrix is defined by

$$
\rho_{\text {red }}\left[\chi_{<}, \chi_{<}^{\prime}, \eta\right]=\int \mathcal{D} \chi_{>} \rho\left[\chi_{<}, \chi_{>}, \chi_{<}^{\prime}, \chi_{>}, \eta\right] .
$$

and evolves in time according to

$$
\rho_{r}\left[\chi_{<f}, \chi_{<f}^{\prime}, \eta\right]=\int d \chi_{<i} \int d \chi_{<i}^{\prime} J_{r}\left[\chi_{<f}, \chi_{<f}^{\prime}, \eta \mid \chi_{<i}, \chi_{<i}^{\prime}, \eta_{0}\right] \rho_{r}\left[\chi_{<i} \chi_{<i}^{\prime}, \eta_{0}\right],
$$

where $J_{r}\left[t, \eta_{0}\right]$ is the reduced evolution operator 


$$
J_{r}\left[\chi_{<f}, \chi_{<f}^{\prime}, \eta \mid \chi_{<i}, \chi_{<i}^{\prime}, \eta_{0}\right]=\int_{\chi_{<i}}^{\chi_{<f}} \mathcal{D} \chi_{<} \int_{\chi_{<i}^{\prime}}^{\chi_{<f}^{\prime}} \mathcal{D} \chi_{<}^{\prime} \exp \frac{i}{\hbar}\left\{S\left[\chi_{<}\right]-S\left[\chi_{<}^{\prime}\right]\right\} F\left[\chi_{<}, \chi_{<}^{\prime}\right]
$$

The influence functional (or Feynman-Vernon functional) $F\left[\chi_{<}, \chi_{<}^{\prime}\right]$ is defined as

$$
\begin{aligned}
& F\left[\chi_{<}, \chi_{<}^{\prime}\right]=\int d \chi_{>i} \int d \chi_{>i}^{\prime} \rho_{>}\left[\chi_{>i}, \chi_{>i}^{\prime}, \eta_{0}\right] \int d \chi_{>f} \int_{\chi_{>i}}^{\chi_{>f}} \mathcal{D} \chi_{>} \int_{\chi_{>i}^{\prime}}^{\chi_{>f}} \mathcal{D} \chi_{>}^{\prime} \\
& \times \exp \frac{i}{\hbar}\left\{S\left[\chi_{>}\right]+S_{\text {int }}\left[\chi_{<}, \chi_{>}\right]-S\left[\chi_{>}^{\prime}\right]-S_{\text {int }}\left[\chi_{<}^{\prime}, \chi_{>}^{\prime}\right]\right\} .
\end{aligned}
$$

We see that when the environment is initially in its vacuum state, the influence functional coincides with the CTP CGEA 59,7]. Treatment of two self-interacting fields via the influence functional leading to noise is contained in 8

As the propagator $J_{r}$ is defined through a path integral, we can obtain an estimation using the saddle point approximation:

$$
J_{r}\left[\chi_{<f}, \chi_{<f}^{\prime}, \eta \mid \chi_{<i}, \chi_{<i}^{\prime}, \eta_{0}\right] \approx \exp \frac{i}{\hbar} S_{\Lambda_{c}}\left[\chi_{<}^{c l}, \chi_{<}^{\prime c l}\right]
$$

where $\chi_{<}^{c l}\left(\chi_{<}^{\prime c l}\right)$ is the solution of the equation of motion $\left.\frac{\delta R e S_{\Lambda_{c}}}{\delta \chi_{<}}\right|_{\chi_{<}=\chi_{<}^{\prime}}=0$ with boundary conditions $\chi_{<}^{c l}\left(\eta_{0}\right)=\chi_{<i}\left(\chi_{<i}^{\prime}\right)$ and $\chi_{<}^{c l}(\eta)=\chi_{<f}\left(\chi_{<f}^{\prime}\right)$. This formula enables us to analyze the quantum to classical transition of the system field using the perturbative evaluation of the CTP CGEA described in the previous section.

\section{A. Master equation and decoherence of long wavelengths}

The decoherence effects are contained in the imaginary part of the CTP CGEA, which is already of order $\lambda^{2}$. As a consequence, in the evaluation of $\operatorname{Im} S_{\Lambda_{c}}$ we can approximate $\chi_{<}^{c l}$ by the solution of the free field equation satisfying the appropriate boundary conditions. For simplicity we will consider massless, conformally coupled fields. Therefore the classical solution reads

$$
\chi_{<}^{c l}(\vec{x}, s)=\left[\chi_{<f} \frac{\sin \left(k_{0} s\right)}{\sin \left(k_{0} \eta\right)}+\chi_{<i} \frac{\sin \left[k_{0}(\eta-s)\right]}{\sin \left(k_{0} \eta\right)}\right] \cos \left(\vec{k}_{0} \cdot \vec{x}\right) \equiv \chi_{<}^{c l}(s) \cos \left(\vec{k}_{0} \cdot \vec{x}\right),
$$

where we assumed that the system-field contains only one Fourier mode with $\vec{k}=\vec{k}_{0}$. This is a sort of "minisuperspace" approximation for the system-field that will greatly simplify the calculations.

As in the QBM problem, we can derive the diffusion coefficients from the master equation, which would provide relevant information about decoherence. $\mathrm{Hu}$ Paz and Zhang [47,6] derived the noise kernel of two interacting $\phi^{4}$ quantum fields in de Sitter space and analyzed its behavior in relation to decoherence. Lombardo and Mazzitelli [50] did the same, with one self-interacting field split into two mode sectors. They followed the method proposed in [60, and computed the time derivative of the propagator $J_{r}$, eliminating the dependence on the initial field configurations $\chi_{<i}$ and $\chi_{<i}^{\prime}$ that enters through $\chi_{<}^{c l}$ and $\chi_{<}^{\prime c l}$. The master equation is of the form

$$
\begin{aligned}
& i \hbar \partial_{\eta} \rho_{r}\left[\chi_{<f}, \chi_{<f}^{\prime}, \eta\right]=\left\langle\chi_{<f}\left|\left[\hat{H}_{r e n}, \hat{\rho}_{r}\right]\right| \chi_{<f}^{\prime}\right\rangle-i \lambda^{2}\left[\frac{\left(\chi_{<f}^{3}-\chi_{<f}^{\prime 3}\right)^{2} V}{1152} D_{1}\left(k_{0} ; \eta\right)\right. \\
& \left.+\frac{\left(\chi_{<f}^{2}-\chi_{<f}^{\prime 2}\right)^{2} V}{32} D_{2}\left(k_{0} ; \eta\right)-\frac{\left(\chi_{<f}-\chi_{<f}^{\prime}\right)^{2} V}{6} D_{3}\left(k_{0} ; \eta\right)\right] \rho_{r}\left[\chi_{<f}, \chi_{<f}^{\prime}, \eta\right]+\ldots
\end{aligned}
$$

Due to the complexity of the equation, we only show the correction to the usual unitary evolution term coming from the noise kernels.

This equation contains three time-dependent diffusion coefficients $D_{i}(\eta)$. Up to one loop, only $D_{1}$ and $D_{2}$ survive and are given by 


$$
\begin{aligned}
& D_{1}\left(k_{0} ; \eta\right)=\int_{0}^{t} d s \cos ^{3}\left(k_{0} s\right) \operatorname{Im} G_{++}^{\Lambda_{c}}\left(3 k_{0} ; \eta-s\right) \\
&= \frac{1}{6 k_{0}} \int_{0}^{t} d s \cos ^{3}\left(k_{0} s\right) \cos \left(3 k_{0} s\right) \theta\left(3 k_{0}-\Lambda_{c}\right) \\
&= \frac{2 k_{0} \eta+3 \sin \left(2 k_{0} \eta\right)+\frac{3}{2} \sin \left(4 k_{0} \eta\right)+\frac{1}{3} \sin \left(6 k_{0} \eta\right)}{576 k_{0}^{2}}, \quad \frac{\Lambda_{c}}{3}<k_{0}<\Lambda_{c} \\
& D_{2}\left(k_{0} ; \eta\right)=\int_{0}^{\eta} d s \cos ^{2}\left(k_{0} s\right)\left(\operatorname{Re} G_{++}^{\Lambda_{c} 2}\left(2 k_{0} ; \eta-s\right)+2 \operatorname{Re} G_{++}^{\Lambda_{c} 2}(0 ; \eta-s)\right) .
\end{aligned}
$$

Using that

$$
\begin{aligned}
\operatorname{Re} G_{++}^{\Lambda_{c} 2}\left(2 k_{0} ; \eta-s\right)= & \frac{\pi}{k_{0}}\left\{\int_{\Lambda_{c}}^{2 k_{0}+\Lambda_{c}} d p \int_{\Lambda_{c}}^{2 k_{0}+p} d z \cos [(p+z) s]\right. \\
& \left.+\int_{2 k_{0}+\Lambda_{c}}^{\infty} d p \int_{p-2 k_{0}}^{p+2 k_{0}} d z \cos [(p+z) s]\right\} \\
\operatorname{Re} G_{++}^{\Lambda_{c} 2}(0 ; \eta-s) & =\pi\left\{2 \pi \delta(s)-2 \frac{\sin \left(2 \Lambda_{c} s\right)}{s}\right\}
\end{aligned}
$$

the $D_{2}$ diffusion coefficient can be written as

$$
\begin{aligned}
D_{2}\left(k_{0} ; \eta\right)= & \frac{\pi}{4}\left\{3 \pi-\left(\frac{3}{2}-\frac{\Lambda_{c}}{2 k_{0}}\right) \operatorname{Si}\left[2 \eta\left(\Lambda_{c}-k_{0}\right)\right]\right. \\
& -\left(2-\frac{\Lambda_{c}}{2 k_{0}}\right) \operatorname{Si}\left[2 \Lambda_{c} \eta\right]-\left(\frac{3}{2}+\frac{\Lambda_{c}}{2 k_{0}}\right) \operatorname{Si}\left[2 \eta\left(\Lambda_{c}+k_{0}\right)\right]-\left(1+\frac{\Lambda_{c}}{2 k_{0}}\right) \operatorname{Si}\left[2 \eta\left(2 k_{0}+\Lambda_{c}\right)\right] \\
& \left.+\frac{\cos \left[2 \Lambda_{c} \eta\right]}{4 k_{0} t}-\frac{\cos \left[2 \eta\left(\Lambda_{c}+k_{0}\right)\right]}{4 k_{0} \eta}+\frac{\cos \left[2 \eta\left(\Lambda_{c}-k_{0}\right)\right]}{4 k_{0} \eta}-\frac{\cos \left[2 \eta\left(2 k_{0}+\Lambda_{c}\right)\right]}{4 k_{0} \eta}\right\},
\end{aligned}
$$

where $S i[z]$ denotes the sine-integral function $[63]$.

Eq. (VII.12) is the field-theoretical version of the QBM master equation we were looking for. In our case, the system is coupled in a nonlinear form. Owing to the existence of three interaction terms $\left(\chi_{<}^{3} \chi_{>}, \chi_{<}^{2} \chi_{>}^{2}\right.$, and $\chi<\chi_{>}^{3}$ ) there are three diffusion coefficients in the master equation. The form of the coefficients is fixed by these couplings and by the particular choice of the quantum state of the environment.

Our results are valid in the single-mode approximation of Eq. (VII.11). In this approximation one obtains a reduced density matrix for each mode $\vec{k}_{0}$, and neglects the interaction between different system-modes. Due to this interaction, in the general case, $\rho_{r}$ will be different from $\prod_{\vec{k}_{0}} \rho_{r}\left(\vec{k}_{0}\right)$. This point deserves further study.

A detailed analysis of the quantum to classical transition in the model we are considering is a very complicated task. One should analyze in detail the master equation and see whether the off-diagonal elements of the reduced density matrix are suppressed or not. One should also study the form of the Wigner function, and see whether it predicts classical correlations or not 64,65] Having in mind the analogy with the QBM, here we will only be concerned with the diffusive terms of the master equation. By examining how the value of the diffusion coefficients change in time, one can get an indication of how effective decoherence is [6, 50]. This simplified method can only provide a rough approximation.

A mode in the system will decohere if the diffusion coefficients are different from zero during an appreciable period of time. Therefore we ask the following question: which is the maximum value of the cutoff $\Lambda_{c}$ such that, a few e-foldings after the initial time, all modes with $k_{0} \leq \Lambda_{c}$ still suffer the diffusive effects? The value of the diffusion coefficients at a given time depends on the value of the adimensional quantity $l=\Lambda_{c} \eta$. For the particular case of a de Sitter spacetime we have $a(t)=\exp (H t)$ and

$$
l=\Lambda_{c} \eta=\frac{\Lambda_{c}}{H}\left(1-\frac{1}{a}\right)
$$


which, a few e-foldings after the initial time, is approximately given by $l=\Lambda_{c} / H$. We can distinguish two possibilities. When $l \sim 1$ both coefficients $D_{1}$ and $D_{2}$ are appreciably different from zero for all values of $k_{0}$. On the other hand, when $l \gg 1$ the situation is completely different: $D_{1}$ is very small everywhere while $D_{2}$ is also very small in the infrared sector. The diffusive effects are important only for $k_{0} \sim \Lambda_{c}$. The conclusion is that, in order to have decoherence for all modes with $k_{0}<\Lambda_{c}$, we can include in our system only those modes with wavelength larger than $H^{-1}$. This is consistent with Starobinsky's original suggestion. If we include wavelengths shorter than $H^{-1}$, the frequency threshold in the environment increases, the infrared sector of the system cannot excite the environment and therefore does not decohere.

A more realistic calculation should include a time dependent cutoff [66], since the system should contain, at each time, the modes with $k_{p h}=\frac{k_{0}}{a}<H$. More importantly, the scalar field should be minimally coupled $(\xi=0)$ to the curvature. In spite of this, we think that our example illustrates the main aspects of the problem. Indeed, one could repeat the calculations for a given mode of the minimally coupled scalar field, and obtain a master equation, similar to our Eq. (VII.12). The main difference would be that, for $\xi=0$, the propagators in De Sitter spacetime would differ from their flat-spacetime counteparts Eqs. (VI.13 VI.15) (See Zhang's thesis [47). The evaluation of the diffusion coefficients using curved spacetime propagators would be more realistic for discussions of structure formation in inflationary cosmology.

\section{B. The Langevin equation}

We now show how to derive a stochastic (Langevin) equation for the system field from the CTP CGEA. This equation takes into account the three fundamental effects of the environment on the system: renormalization, dissipation and noise.

The real and imaginary parts of the CTP CGEA for our model are given in Eqs. (VI.19) and (VI.20). One can regard the imaginary part of $S_{\Lambda_{c}}$ as coming from three noise sources $\nu(x), \xi(x)$, and $\eta(x)$ with a Gaussian functional probability distribution given by

$$
\begin{aligned}
& P[\nu(x), \xi(x), \eta(x)]=N_{\nu} N_{\xi} N_{\eta} \exp \left\{-\frac{1}{2} \int d^{4} x \int d^{4} y \nu(x)\left[\frac{\lambda^{2}}{9} \operatorname{Im} G_{++}^{\Lambda_{c}}\right]^{-1} \nu(y)\right\} \\
& \left.\times \exp \left\{-\frac{1}{2} \int d^{4} x \int d^{4} y \xi(x)\left[\frac{\lambda^{2}}{2} \operatorname{Re} G_{++}^{\Lambda_{c} 2}\right]^{-1}\right] \xi(y)\right\} \\
& \times \exp \left\{-\frac{1}{2} \int d^{4} x \int d^{4} y \eta(x)\left[\frac{-2 \lambda^{2}}{3} \operatorname{Im} G_{++}^{\Lambda_{c} 3}\right]^{-1} \eta(y)\right\},
\end{aligned}
$$

where $N_{\nu}, N_{\xi}$, and $N_{\eta}$ are normalization factors. Indeed, we can write the imaginary part of the influence action as three functional integrals over the Gaussian fields $\nu(x), \xi(x)$, and $\eta(x)$ :

$$
\begin{aligned}
& \int \mathcal{D} \nu(x) \int \mathcal{D} \xi(x) \int \mathcal{D} \eta(x) P[\nu, \xi, \eta] \exp -\frac{i}{\hbar}\left\{R_{-}(x) \nu(x)+Q_{-}(x) \xi(x)+\chi_{-}(x) \eta(x)\right\} \\
& =\exp \left\{-\frac{i}{\hbar} \int d^{4} x \int d^{4} y\left[\frac{\lambda^{2}}{18} R_{-}(x) \operatorname{Im} G_{++}^{\Lambda}(x, y) R_{-}(y)\right.\right. \\
& \left.\left.+\frac{\lambda^{2}}{4} Q_{-}(x) R e G_{++}^{\Lambda 2}(x, y) Q_{-}(y)-\frac{\lambda^{2}}{3} \chi_{-}(x) \operatorname{Im} G_{++}^{\Lambda 3}(x, y) \chi_{-}(y)\right]\right\}
\end{aligned}
$$

Therefore, the CTP-CGEA can be rewritten as

$$
S_{\Lambda_{c}}\left[\chi_{<}, \chi_{<}^{\prime}\right]=-\frac{1}{i} \ln \int \mathcal{D} \nu P[\nu] \int \mathcal{D} \xi P[\xi] \int \mathcal{D} \eta P[\eta] \exp \left\{i S_{e f f}\left[\chi_{<}, \chi_{<}^{\prime}, \nu, \xi, \eta\right]\right\},
$$

where

$$
S_{e f f}\left[\chi_{<}, \chi_{<}^{\prime}, \nu, \xi, \eta\right]=R e S_{\Lambda_{c}}\left[\chi_{<}, \chi_{<}^{\prime}\right]-\int d^{4} x\left[R_{-}(x) \nu(x)+Q_{-}(x) \xi(x)+\chi_{-}(x) \eta(x)\right] .
$$

From this effective action it is easy to derive the stochastic field equation for the system 


$$
\left.\frac{\partial S_{e f f}\left[\chi_{<}, \chi_{<}^{\prime}, \nu, \xi, \eta\right]}{\partial \chi_{<}}\right|_{\chi_{<}=\chi_{<}^{\prime}}=0
$$

It is given by

$$
\begin{aligned}
& \square \chi_{<}+\frac{1}{6} \chi_{<}^{3}+\frac{1}{12} \lambda^{2} \chi_{<}^{2}(x) \int d^{4} y \theta\left(x_{0}-y_{0}\right) \operatorname{Re} G_{++}^{\Lambda_{c}}(x, y) \chi_{<}^{3}(y) \\
& +\frac{1}{4} \lambda^{2} \chi_{<}(x) \int d^{4} y \theta\left(x_{0}-y_{0}\right) \operatorname{Im} G_{++}^{\Lambda_{c} 2}(x, y) \chi_{<}^{2}(y)+\frac{1}{6} \lambda^{2} \int d^{4} y \theta\left(x_{0}-y_{0}\right) \operatorname{Re} G_{++}^{\Lambda_{c} 3}(x, y) \chi_{<}(y) \\
& =\frac{3}{2} \nu(x) \chi_{<}^{2}(x)+\xi(x) \chi_{<}(x)+\frac{1}{2} \eta(x) .
\end{aligned}
$$

The field equation is real and causal, as expected. We see that it contains multiplicative and additive colored noise. The non linear coupling between modes makes the Langevin equation much more complicated than the usually assumed white noise equation (see Section III C).

Greiner and Müller [51] obtained a similar stochastic equation in flat spacetime, for a thermal environment. They analyzed in detail the dissipative terms in the Langevin equation. In particular they found explicit expressions for momentum dependent dissipation coefficients using a Markovian approximation for the soft modes.

\section{RENORMALIZATION GROUP FROM CTP CGEA}

\section{A. Towards a nonperturbative evaluation of the CTP CGEA: The exact RG equation}

The main drawback of the results presented in the previous sections is that we have evaluated the CTP CGEA only perturbatively. As is well known, several applications, in particular the analysis of phase transitions in the early universe and condensed matter physics require nonperturbative calculations. In this section we will derive an exact evolution equation for the dependence of the CGEA on the coarse graining scale [52]. In order to simplify the notation in this section we will denote by $\Lambda_{0}$ the ultraviolet cutoff and by $\Lambda$ the coarse graining scale. The CTP CGEA interpolates between the bare theory at $\Lambda=\Lambda_{0}$ and the physical theory at the scale $\Lambda$. We will now consider a $\lambda \phi^{4}$ field theory in Minkowski spacetime.

To derive such an exact evolution equation, we follow the approach of Wegner and Houghton 40] which is designed for Euclidean spacetime. Therefore we will invoke the Euclidean average effective action of Wetterich et al [37,67 71]. (As already mentioned, the main difference between both actions is that the Euclidean action averages the field over a space-time volume, while our CTP CGEA averages the field over a spatial volume, and is therefore more adept to study non-equilibrium conditions.)

The Euclidean CGEA is defined by

$$
e^{-S_{\Lambda}(\phi)} \equiv \int \prod_{\Lambda_{0}>q>\Lambda} \mathcal{D}[\phi(q)] e^{-S_{c l}[\phi]}
$$

By considering an infinitesimal variation $\Lambda \rightarrow \Lambda-\delta \Lambda$ it is possible to obtain an evolution equation for $S_{\Lambda}$, which reads

$$
\Lambda \frac{\partial S_{\Lambda}}{\partial \Lambda}=-\frac{\Lambda}{2 \delta \Lambda} \int^{\prime} \frac{d^{4} q}{(2 \pi)^{4}} \ln \left(\frac{\delta^{2} S_{\Lambda}}{\delta \phi_{q} \delta \phi_{-q}}\right)-\int^{\prime} \frac{d^{4} q}{(2 \pi)^{4}} \frac{\delta S_{\Lambda}}{\delta \phi_{q}} \frac{\delta S_{\Lambda}}{\delta \phi_{-q}}\left(\frac{\delta^{2} S_{\Lambda}}{\delta \phi_{q} \delta \phi_{-q}}\right)^{-1}
$$

(in the original equation of Wegner and Houghton there are additional terms coming from a rescaling of the modes after the coarse graining). The prime in the momenta integrals means that integration is restricted to the shell $\Lambda>q>\Lambda-\delta \Lambda$

A typical approximation taken to solve this evolution equation is to assume that $S_{\Lambda}$ is of the form

$$
S_{\Lambda}[\phi]=\int d^{4} x\left[\frac{1}{2}\left(\partial_{\mu} \phi\right)^{2}+V_{\Lambda}(\phi)\right]
$$

Eq. VIII.2) reduces in this case to an evolution equation for the effective potential $V_{\Lambda}(\phi)$. We will discuss this kind of approximation for the CTP CGEA in the next section. 
Now we start the CTP calculation by writing the CGEA for a scale $\Lambda-\delta \Lambda$, namely

$$
e^{i S_{\Lambda-\delta \Lambda}\left(\phi_{+}, \phi_{-}\right)} \equiv \int \prod_{\Lambda_{0}>|\vec{q}|>\Lambda-\delta \Lambda} \mathcal{D}\left[\phi_{+}(\vec{q}, t)\right] \mathcal{D}\left[\phi_{-}(\vec{q}, t)\right] e^{i S_{c l}\left[\phi_{+}, \phi_{-}\right]}
$$

The modes to be integrated can be split into two parts: one within the shell $\Lambda>|\vec{q}|>\Lambda-\delta \Lambda$ and another containing modes with $\Lambda_{0}>|\vec{q}|>\Lambda$. Expanding the action in powers of the modes within the shell, one obtains

$$
\begin{aligned}
e^{i S_{\Lambda-\delta \Lambda}\left(\phi_{+}, \phi_{-}\right)}= & e^{i S_{\Lambda}\left(\phi_{+}, \phi_{-}\right)} \times \\
& \int \prod_{\Lambda>|\vec{q}|>\Lambda-\delta \Lambda} \mathcal{D}\left[\phi_{+}\right] \mathcal{D}\left[\phi_{-}\right] e^{i\left(S_{1}+S_{2}+S_{3}\right)} e^{\frac{i}{2} \int \frac{d^{3} q}{(2 \pi)^{3}} \int d t \frac{d}{d t}\left(\phi_{a}(-\vec{q}, t) \dot{\phi}_{b}(\vec{q}, t) g_{a b}\right)}
\end{aligned}
$$

where

$$
\begin{aligned}
& S_{1}=\int d t \int^{\prime} \frac{d^{3} q}{(2 \pi)^{3}} \phi_{a}(\vec{q}, t) \frac{\partial S_{\Lambda}}{\partial \phi_{a}(-\vec{q}, t)} \\
& S_{2}=\frac{1}{2} \int d t d t^{\prime} \int^{\prime} \frac{d^{3} q}{(2 \pi)^{3}} \phi_{a}(\vec{q}, t) \frac{\partial^{2} S_{\Lambda}}{\partial \phi_{a}(-\vec{q}, t) \phi_{b}\left(\vec{q}, t^{\prime}\right)} \phi_{b}\left(\vec{q}, t^{\prime}\right) .
\end{aligned}
$$

In taking the functional derivatives of $S_{\Lambda}$ (which contains modes whose wave vectors satisfy $|\vec{q}|<\Lambda$ ) the modes within the shell are set to zero. We use the notation

$$
\phi_{a}(\vec{q}, t)=\left(\begin{array}{c}
\phi_{+}(\vec{q}, t) \\
\phi_{-}(\vec{q}, t)
\end{array}\right) ; g_{a b}=\left(\begin{array}{cc}
1 & 0 \\
0 & -1
\end{array}\right)
$$

The $S_{3}$ term is cubic in the modes within the shell and, as in the Euclidean case, it does not contribute in the limit $\delta \Lambda \rightarrow 0$ (basically, this is because one is doing a one loop calculation for the shell modes). The functional integrals over the shell modes have the CTP boundary conditions. A comment about the last exponential factor in Eq. (VIII.5) is in order. Usually one discards it because it is a surface term, but in the CTP formalism it must be kept since the boundary conditions are that $\phi_{+}(\vec{q}, T)=\phi_{-}(\vec{q}, T)$ with $T \rightarrow \infty$ for the modes $\vec{q}$ within the shell.

In order to evaluate the functional integrals we split the field as $\phi_{a}=\bar{\phi}_{a}+\varphi_{a}$ and impose the boundary conditions on the "classical" fields $\bar{\phi}_{ \pm}$, i.e. they vanish in the past $-T$ (negative and positive frequencies respectively) and match in the Cauchy surface at time $T$. The fluctuations $\varphi_{a}$ vanish both in the past and in the future. The classical fields are solutions to

$$
\left(-\frac{d^{2}}{d t^{2}}-q^{2}\right) g_{a b} \bar{\phi}_{b}(\vec{q}, t)+\int d t^{\prime} \frac{\partial^{2} S_{i n t}}{\partial \varphi_{a}(-\vec{q}, t) \partial \varphi_{b}\left(\vec{q}, t^{\prime}\right)} \bar{\phi}_{b}\left(\vec{q}, t^{\prime}\right)=0
$$

where we have split the CGEA as $S_{\Lambda}\left(\phi_{ \pm}\right)=S_{k i n}\left(\phi_{ \pm}\right)+S_{i n t}\left(\phi_{ \pm}\right)$with

$$
S_{k i n}=\int d^{4} x\left[\frac{1}{2}\left(\partial_{\mu} \phi_{+}\right)^{2}+\frac{i \epsilon}{2} \phi_{+}^{2}\right]-\int d^{4} x\left[\frac{1}{2}\left(\partial_{\mu} \phi_{-}\right)^{2}-\frac{i \epsilon}{2} \phi_{-}^{2}\right]
$$

As before, in the functional derivatives the modes within the shell are set to zero.

Let $h_{a}$ be solutions to Eq.(VIII.8), vanishing in the past and satisfying an arbitrary normalization in the future, and let $\phi(\vec{q})$ be the common value of the fields taken in the future. We can then write

$$
\bar{\phi}_{a}(\vec{q}, t)=\phi(\vec{q}) \frac{h_{a}(\vec{q}, t)}{h_{a}(\vec{q}, T)}
$$

We first integrate over the common value $\phi(\vec{q})$ and then proceed with the functional integration over the fluctuations $\varphi_{a}$ (both are Gaussian integrals with "source" terms). One finally gets 


$$
\begin{aligned}
\Lambda \frac{\partial S_{\Lambda}}{\partial \Lambda}= & -\frac{i \Lambda}{2 \delta \Lambda} \int^{\prime} \frac{d^{3} q}{(2 \pi)^{3}} \ln \left(\frac{\dot{h}_{+}(\vec{q}, T)}{h_{+}(\vec{q}, T)}-\frac{\dot{h}_{-}(\vec{q}, T)}{h_{-}(\vec{q}, T)}\right)+ \\
& \frac{\Lambda}{2 \delta \Lambda} \int^{\prime} \frac{d^{3} q}{(2 \pi)^{3}}\left(\frac{\dot{h}_{+}(\vec{q}, T)}{h_{+}(\vec{q}, T)}-\frac{\dot{h}_{-}(\vec{q}, T)}{h_{-}(\vec{q}, T)}\right)^{-1}\left(\int d t \frac{h_{a}(\vec{q}, t)}{h_{a}(\vec{q}, T)} \frac{\partial S_{\Lambda}}{\partial \varphi_{a}(-\vec{q}, t)}\right)^{2}- \\
& \frac{i \Lambda}{2 \delta \Lambda} \ln \operatorname{det}^{\prime}\left(A_{a b}\right)+ \\
& \frac{\Lambda}{2 \delta \Lambda} \int d t d t^{\prime} \int^{\prime} \frac{d^{3} q}{(2 \pi)^{3}} \frac{\partial S_{\Lambda}}{\partial \varphi_{a}(\vec{q}, t)} A_{a b}^{-1}\left(-\vec{q}, t ; \vec{q}, t^{\prime}\right) \frac{\partial S_{\Lambda}}{\partial \varphi_{b}\left(\vec{q}, t^{\prime}\right)}
\end{aligned}
$$

The $2 \times 2$ matrix $A_{a b}$ has the following elements

$$
\begin{aligned}
& A_{++}\left(-\vec{q}, t ; \vec{q}^{\prime}, t^{\prime}\right)=\left(-\frac{d^{2}}{d t^{2}}-q^{2}+i \epsilon\right) \delta\left(t-t^{\prime}\right) \delta^{3}\left(\vec{q}+\vec{q}^{\prime}\right)+\frac{\partial^{2} S_{i n t}}{\partial \varphi_{+}(-\vec{q}, t) \partial \varphi_{+}\left(\vec{q}^{\prime}, t^{\prime}\right)} \\
& A_{--}\left(-\vec{q}, t ; \vec{q}^{\prime}, t^{\prime}\right)=\left(\frac{d^{2}}{d t^{2}}+q^{2}+i \epsilon\right) \delta\left(t-t^{\prime}\right) \delta^{3}\left(\vec{q}+\vec{q}^{\prime}\right)+\frac{\partial^{2} S_{i n t}}{\partial \varphi_{-}(-\vec{q}, t) \partial \varphi_{-}\left(\vec{q}^{\prime}, t^{\prime}\right)} \\
& A_{+-}\left(-\vec{q}, t ; \vec{q}^{\prime}, t^{\prime}\right)=A_{-+}\left(\vec{q}^{\prime}, t^{\prime} ;-\vec{q}, t\right)=\frac{\partial^{2} S_{i n t}}{\partial \varphi_{+}(-\vec{q}, t) \partial \varphi_{-}\left(\vec{q}^{\prime}, t^{\prime}\right)}
\end{aligned}
$$

The primed determinant must be calculated as the product of the eigenvalues of $A_{a b}$ in a space of functions with wave vectors within the shell $(\Lambda-\delta \Lambda<|\vec{q}|<\Lambda)$ and satisfying null conditions both in the past and in the future. Similar conditions are to be used to evaluate the inverse $A_{a b}^{-1}$.

The equation (VIII.11) is exact in the sense that no perturbative approximation has so far been used. It is similar to its Euclidean counterpart (VIII.2), but involves two fields and CTP boundary conditions. It contains all the information of the influence of the short wavelength modes on the long wavelength ones, and should be the starting point for a non-perturbative analysis of decoherence, dissipation, domain formation and out of equilibrium evolution.

\section{B. Derivative expansion}

The exact renormalization group equation is too complex to be solved without taking some approximation. The usual ones are expansions in the number of powers of the fields (see Ref. [72] for a detailed analysis) or in derivatives of them 73 75]. In the following we shall make use of the derivative expansion approach.

We will prove that, within this approach, the exact RG Eq.(VIII.11) admits a solution of the form

$$
S_{\Lambda}\left(\phi_{+}, \phi_{-}\right)=S_{\Lambda}\left(\phi_{+}\right)-S_{\Lambda}\left(\phi_{-}\right)
$$

Clearly this is not the most general form that can be imagined for the coarse grained action because contributions involving mixing of both fields are not taken into account. The main drawback of this approach is therefore that we miss the stochastic aspects of the theory, since there can be no noise terms. However, the proposed form for the CGEA will be enough for studying the renormalization group flow of real time field theories.

The great technical advantage of the form Eq. (VIII.13) is that the second functional derivative of the action has no crossed terms, leading to a diagonal matrix $A_{a b}$ whose determinant is easily computed as the product of two determinants, one for $A_{++}$and one for $A_{--}$. Following Ref. [76] one can express both $\operatorname{det}^{\prime} A_{++}$and $\operatorname{det}^{\prime} A_{--}$as the product over momenta of a constant (momenta independent) times the mode $h(\vec{q}, T)$ evaluated at the final time $T$. Therefore the last term of the exact RG equation can be written as

$$
\ln \operatorname{det}^{\prime}\left(A_{a b}\right)=\ln \left[\operatorname{det}^{\prime}\left(A_{++}\right) \operatorname{det}^{\prime}\left(A_{--}\right)\right]=\int^{\prime} \frac{d^{3} q}{(2 \pi)^{3}} \ln \left(h_{+}(\vec{q}, T) h_{-}(\vec{q}, T)\right)
$$

The first and the third terms can then be cast in the form of a single logarithm, and we arrive at 


$$
\begin{aligned}
\Lambda \frac{\partial S_{\Lambda}}{\partial \Lambda}= & -\frac{i \Lambda}{2 \delta \Lambda} \int^{\prime} \frac{d^{3} q}{(2 \pi)^{3}} \ln \left(h_{-}(\vec{q}, T) \dot{h}_{+}(\vec{q}, T)-h_{+}(\vec{q}, T) \dot{h}_{-}(\vec{q}, T)\right)+ \\
& \frac{\Lambda}{2 \delta \Lambda} \int^{\prime} \frac{d^{3} q}{(2 \pi)^{3}}\left(\frac{\dot{h}_{+}(\vec{q}, T)}{h_{+}(\vec{q}, T)}-\frac{\dot{h}_{-}(\vec{q}, T)}{h_{-}(\vec{q}, T)}\right)^{-1}\left(\int d t \frac{h_{a}(\vec{q}, t)}{h_{a}(\vec{q}, T)} \frac{\partial S_{\Lambda}}{\partial \varphi_{a}(-\vec{q}, t)}\right)^{2}+ \\
& \frac{\Lambda}{2 \delta \Lambda} \int d t d t^{\prime} \int^{\prime} \frac{d^{3} q}{(2 \pi)^{3}} \frac{\partial S_{\Lambda}}{\partial \varphi_{a}(\vec{q}, t)} A_{a b}^{-1}\left(-\vec{q}, t ; \vec{q}, t^{\prime}\right) \frac{\partial S_{\Lambda}}{\partial \varphi_{b}\left(\vec{q}, t^{\prime}\right)}
\end{aligned}
$$

Note that the equations for the two modes $h_{+}$and $h_{-}$(Eq. (VIII.8)) simplify considerably, since the two equations are decoupled. What we still have to prove is that the proposed form for the action makes the r.h.s. of the exact RG equation split in the same form.

Next we perform a derivative expansion of the interaction term. As our coarse graining explicitly breaks Lorentz invariance, we allow different coefficients for the temporal and spatial derivatives, namely

$$
S_{i n t}\left(\phi_{ \pm}\right)=\int d^{4} x\left[-V_{\Lambda}\left(\phi_{ \pm}\right)+\frac{1}{2} Z_{\Lambda}\left(\phi_{ \pm}\right) \dot{\phi}_{ \pm}^{2}-\frac{1}{2} Y_{\Lambda}\left(\phi_{ \pm}\right)\left(\vec{\nabla} \phi_{ \pm}\right)^{2}+\ldots\right]
$$

We expand the fields around a time dependent background: $\phi_{ \pm}=\phi_{ \pm}(t)+\varphi_{ \pm}(\vec{x}, t)$ and Fourier transform in space. We shall solve the Eq. (VIII.8) for the modes to zeroth order in the inhomogeneities, i.e. we equate terms in the equations for $h_{ \pm}$that are independent of $\varphi_{ \pm}$'s. Since the first functional derivative of the CGEA (S') is linear in the inhomogeneities $\varphi_{ \pm}$, we put $S^{\prime}=0$ and keep the $\varphi_{ \pm}$-independent contributions to $S_{\text {int }}^{\prime \prime}$. After a little algebra and functional derivations, we get

$$
\begin{aligned}
\frac{\partial^{2} S_{i n t}}{\partial \varphi(\vec{q}, t) \partial \varphi\left(-\vec{q}^{\prime}, t^{\prime}\right)}= & {\left[-V^{\prime \prime}-\frac{1}{2} \dot{\phi}^{2} Z^{\prime \prime}-Y q^{2}-Z^{\prime} \dot{\phi} \frac{d}{d t}-Z \frac{d^{2}}{d t^{2}}-\ddot{\phi} Z^{\prime}+\ldots\right] \times } \\
& \delta\left(t-t^{\prime}\right) \delta^{3}\left(\vec{q}-\vec{q}^{\prime}\right)
\end{aligned}
$$

where the primes denote derivation with respect to the field and the ellipsis denote terms linear in the fluctuations. In this expression and hereafter we omit (unless explicitly stated) the \pm subscripts in the background fields $\phi_{ \pm}(t)$, in the potential $V_{\Lambda}\left(\phi_{ \pm}(t)\right)$, and in the wave function factors $Z_{\Lambda}\left(\phi_{ \pm}(t)\right)$ and $Y_{\Lambda}\left(\phi_{ \pm}(t)\right)$. Note that the effective mass of the modes depends on the time-dependent background $\phi(t)$. The equations of motion for the modes $h_{a}$ become localized and take the form of harmonic oscillators with variable frequency and a damping term. The boundary conditions to be imposed are the aforementioned CTP ones.

If one defines new modes as $f(\vec{q}, t)=\left(1+Z_{\Lambda}\right)^{1 / 2} h(\vec{q}, t)$, the damping terms cancel out and the new modes are harmonic oscillators with frequency

$$
w_{q}^{2}(t)=q^{2} \frac{1+Y_{\Lambda}}{1+Z_{\Lambda}}+\frac{V_{\Lambda}^{\prime \prime}}{1+Z_{\Lambda}}+\frac{1}{4} \frac{Z_{\Lambda}^{\prime 2}}{\left(1+Z_{\Lambda}\right)^{2}} \dot{\phi}^{2}+\frac{1}{2} \frac{Z_{\Lambda}^{\prime}}{1+Z_{\Lambda}} \ddot{\phi}
$$

Using an adiabatic expansion for the modes,

$$
h_{ \pm}(\vec{q}, t)=\left(1+Z_{\Lambda}\right)^{-1 / 2} \frac{1}{\sqrt{2 W_{ \pm}(\vec{q}, t)}} e^{ \pm i \int_{-T}^{t} W_{ \pm}\left(\vec{q}, t^{\prime}\right) d t^{\prime}}
$$

we can easily evaluate the logarithmic term in the r.h.s. of the exact RGE (VIII.15), which is the only term that survives in the approximation we are working. The RGE VIII.15) reduces to

$$
\begin{aligned}
& \Lambda \int d t\left\{\left[-\frac{d V_{\Lambda}\left(\phi_{+}\right)}{d \Lambda}+\frac{1}{2} \frac{d Z_{\Lambda}\left(\phi_{+}\right)}{d \Lambda} \dot{\phi}_{+}^{2}\right]-\left[-\frac{d V_{\Lambda}\left(\phi_{-}\right)}{d \Lambda}+\frac{1}{2} \frac{d Z_{\Lambda}\left(\phi_{-}\right)}{d \Lambda} \dot{\phi}_{-}^{2}\right]\right\}= \\
& \frac{\Lambda}{2 \delta \Lambda} \int^{\prime} \frac{d^{3} q}{(2 \pi)^{3}} \int\left[W_{+}(\vec{q}, t)-W_{-}(\vec{q}, t)\right] d t
\end{aligned}
$$

In the adiabatic expansion, the $W$ 's read

$$
W^{2}=A_{\Lambda}+B_{\Lambda} \dot{\phi}^{2}(t)+C_{\Lambda} \ddot{\phi}(t)
$$

where the coefficients are 


$$
\begin{aligned}
A_{\Lambda} & =\Lambda^{2} \frac{1+Y_{\Lambda}}{1+Z_{\Lambda}}+\frac{V_{\Lambda}^{\prime \prime}}{1+Z_{\Lambda}} \\
B_{\Lambda} & =\frac{Z_{\Lambda}^{\prime 2}}{4\left(1+Z_{\Lambda}\right)^{2}}+\frac{5 A_{\Lambda}^{\prime 2}}{16 A_{\Lambda}^{2}}-\frac{A_{\Lambda}^{\prime \prime}}{4 A_{\Lambda}} \\
C_{\Lambda} & =\frac{Z_{\Lambda}^{\prime}}{2\left(1+Z_{\Lambda}\right)}-\frac{A_{\Lambda}^{\prime}}{4 A_{\Lambda}}
\end{aligned}
$$

Integrating by parts we get

$$
\int d t\left\{-\Lambda \frac{d V_{\Lambda}}{d \Lambda}+\frac{1}{2} \Lambda \frac{d Z_{\Lambda}}{d \Lambda} \dot{\phi}^{2}\right\}=\frac{\Lambda^{3}}{4 \pi^{2}} \int d t\left\{\sqrt{A_{\Lambda}}+\frac{1}{2} \dot{\phi}^{2}\left[\frac{B_{\Lambda}}{\sqrt{A_{\Lambda}}}-\left(\frac{C_{\Lambda}}{\sqrt{A_{\Lambda}}}\right)^{\prime}\right]\right\}
$$

Therefore the dependence of the potential and the wave function renormalization on the infrared scale is given by

$$
\begin{aligned}
& \Lambda \frac{d V_{\Lambda}}{d \Lambda}=-\frac{\Lambda^{3}}{4 \pi^{2}} \sqrt{\Lambda^{2} \frac{1+Y_{\Lambda}}{1+Z_{\Lambda}}+\frac{V_{\Lambda}^{\prime \prime}}{1+Z_{\Lambda}}} \\
& \Lambda \frac{d Z_{\Lambda}}{d \Lambda}=\frac{\Lambda^{3}}{4 \pi^{2}}\left[\frac{B_{\Lambda}}{\sqrt{A_{\Lambda}}}-\left(\frac{C_{\Lambda}}{\sqrt{A_{\Lambda}}}\right)^{\prime}\right]
\end{aligned}
$$

These equations are valid both for the $\phi_{+}$field and for the $\phi_{-}$field.

The above equations describe the flow of the coarse grained action with the infrared scale in the derivative expansion of the exact CTP renormalization group equation. It is interesting to note that the higher derivative terms modify the differential equation for the effective potential.

We have obtained two equations for the three independent unknown functions $V_{\Lambda}, Z_{\Lambda}$ and $Y_{\Lambda}$. In order to find an additional relation between the spatial and temporal wave funtion renormalization functions $Z_{\Lambda}$ and $Y_{\Lambda}$, it is necessary to write the exact RGE up to quadratic order in the inhomogeneities. We will not present this long calculation here. For simplicity, we will assume that $Z_{\Lambda}$ and $Y_{\Lambda}$ are small numbers, and therefore we will set them to zero on the r.h.s. of Eq.(VIII.24). This assumption is partially confirmed by numerical calculations [52]. Note that in this approximation we recover the RG improved equation proposed in Ref. [77 for the coarse grained effective potential

$$
\Lambda \frac{d V_{\Lambda}}{d \Lambda}=-\frac{\Lambda^{3}}{4 \pi^{2}} \sqrt{\Lambda^{2}+V_{\Lambda}^{\prime \prime}} .
$$

There are other points which are worth noting. First, when we substitute $V_{\Lambda}, Z_{\Lambda}$ and $Y_{\Lambda}$ by their classical values $V_{\Lambda}=V, Z_{\Lambda}=Y_{\Lambda}=0$, on the r.h.s. of both equations we obtain the one loop evolution equations [52]. Second, while in the one loop approximation it is possible to take the limit $\Lambda_{0} \rightarrow \infty$ (the infinities can be absorbed into the bare mass and coupling constant), in this non-perturbative calculation it is not possible to renormalize the theory (as is the case for Hartree, Gaussian and $1 / N$ approximations). For these reasons we keep $\Lambda_{0}$ as a large (compared with the mass) but finite number.

Once the functions $V_{\Lambda}$ and $Z_{\Lambda}$ are known, one can write the effective dynamical equations for the coarse grained field.

\section{PART FOUR: Renormalization Group in Semiclassical Gravity}

\section{RENORMALIZATION GROUP AND STOCHASTIC SEMICLASSICAL GRAVITY}

In this Section we will describe a different relation between the RG and the CTP CGEA. We will consider the backreaction of quantum matter fields on the spacetime geometry, assumed classical. To analyze this problem, we will use the formulation of quantum open systems and compute the CTP CGEA using an expansion in powers of the spacetime curvature and discuss its relation with the RG equations. 
The usual approach to analyze backreaction in semiclassical gravity is based on the use of the Semiclassical Einstein Equations (SEEs) [78

$$
\frac{1}{8 \pi G}\left[R_{\mu \nu}-\frac{1}{2} R g_{\mu \nu}\right]-\alpha H_{\mu \nu}^{(1)}-\beta H_{\mu \nu}^{(2)}=T_{\mu \nu}^{c l a s}+<T_{\mu \nu}>.
$$

In the SEEs, which can be derived from the real part of the CTP CGEA [1,2,39], the effect of quantum matter fields is taken into account by including as a source the quantum mean value of the energy-momentum tensor. The terms proportional to

$$
H_{\mu \nu}^{(1)}=\left[4 R^{; \mu \nu}-4 g^{\mu \nu} \square R\right]+O\left(R^{2}\right),
$$

and

$$
H_{\mu \nu}^{(2)}=\left[4 R^{\mu \alpha ; \nu} ; \alpha-2 \square R^{\mu \nu}-g^{\mu \nu} \square R\right]+O\left(R^{2}\right),
$$

come from terms quadratic in the curvature in the gravitational action, which are needed to renormalize the theory.

These equations cannot provide a full description of the problem [3], since they do not take into account the fluctuations of the energy momentum tensor around its mean value. The fluctuations can be incorporated by including an additional stochastic term [7, 79,80] on the right hand side of Eq. [X.1. This noise-term can be derived from the imaginary part of the CTP CGEA, in the same way we proceeded for the soft modes of the scalar field (see Section VII.B). When it is incorporated in the SEE, one obtains the "Einstein-Langevin Equations" (ELEs), which include both the dissipative and diffusive effects of the quantum matter on the geometry of spacetime [7], in complete analogy with what happens in quantum Brownian motion typical of quantum open systems. For a review of the semiclassical stochastic gravity program based on the ELEs, see [81.

The ELEs have been derived for arbitrary small metric perturbations conformally coupled to a massless quantum scalar field in a spatially flat background [80], and, in a cosmological setting, for a massive field in a spatially flat Friedmann-Robertson-Walker universe [82], and in a Bianchi type-I spacetime [83]. Further ellaboration of its physical meaning can be found in the papers by Verdaguer and Roura [84]. In ref. [85] it is proven that the ELE may be used to compute certain quantum averages, even in conditions where there is no decoherence.

Here we present the derivation of the ELEs based on the renormalization group equations of Lombardo and Mazzitelli [86]. Using a covariant expansion in powers of the curvature we will see that, for massless quantum fields, the ELEs are determined to leading order by the running couplings of the theory.

This example will show an interesting connection between the "usual" renormalization group and the different versions of the effective action. Indeed, in a naive "Wilsonian" approach, some quantum effects can be taken into account by replacing the coupling constants of the theory by their running counterparts. However, the (usual) running of coupling constants is defined in momentum space, and a careful implementation of the Wilsonian approach leads to a nonlocal effective action in configuration space. We will compute explicitly this non local effective action first in its Euclidean version, and show that it coincides with calculations based on resummations of the Schwinger DeWitt expansion. Using general relations between Euclidean propagators and CTP propagators, we will be able to obtain the CTP CGEA from the Euclidean effective action. From the CTP CGEA we will derive the ELEs, thus showing that they are a consequence of usual renormalization group equations of the theory. As an application, we will compute the leading quantum corrections to the Newtonian potential.

Consider a quantum scalar field on a classical, Euclidean curved background. The classical action is given by

$$
S=S_{\text {grav }}+S_{\text {matter }}
$$

where

$$
S_{\text {grav }}=-\int d^{4} x \sqrt{g}\left[\frac{1}{16 \pi G_{0}}\left(R-2 \Lambda_{0}\right)+\alpha_{0} R^{2}+\beta_{0} R_{\mu \nu} R^{\mu \nu}\right],
$$

and 


$$
S_{\text {matter }}=\frac{1}{2} \int d^{4} x \sqrt{g}\left[\partial_{\mu} \phi \partial^{\mu} \phi+m^{2} \phi^{2}+\xi R \phi^{2}\right] .
$$

Here $\xi$ is the coupling to the curvature. $G_{0}, \Lambda_{0}$, and the dimensionless constants $\alpha_{0}$ and $\beta_{0}$ are bare constants.

The effective action for this theory is a complicated, non local object. It is defined by integrating out the quantum scalar field, that is

$$
e^{-S_{e f f}}=N \int \mathcal{D} \phi e^{-S\left[g_{\mu \nu}, \phi\right]},
$$

where $N$ is a normalization constant. It is in general not possible to find a closed form for it. If we compute it using a covariant expansion in powers of the curvature, the different terms must be constructed with the Riemann tensor and its derivatives $\nabla \nabla \ldots \mathcal{R}$. Using integration by parts and the Gauss Bonnet identity, the effective action can be written only in terms of the Ricci tensor $R_{\mu \nu}$ and $\square R_{\mu \nu}$ [87]. These arguments suggest that the effective action must have the general form

$$
\begin{aligned}
S_{e f f} & =-\int d^{4} x \sqrt{g}\left[\frac{1}{16 \pi G} R+\alpha R^{2}+\beta R_{\mu \nu} R^{\mu \nu}\right] \\
& +\frac{1}{32 \pi^{2}} \int d^{4} x \sqrt{g}\left[F_{0} R+R F_{1}(\square) R+R_{\mu \nu} F_{2}(\square) R^{\mu \nu}+\ldots\right],
\end{aligned}
$$

where the ellipsis denote terms cubic in the curvature. For simplicity, in the above equation and in what follows we will omit the cosmological constant term. Note that the bare constants in Eq. ([X.5) have been replaced by dressed couplings in Eq. (IX.8). The expansion is adequate for weak gravitational fields, i.e. $\nabla \nabla \mathcal{R} \ll \mathcal{R}^{2}$.

Up to this order, all the information about the effect of the quantum field is encoded in the constant $F_{0}$ and in the form factors $F_{1}$ and $F_{2}$. The form factors are, in general, non-local two point functions constructed with the d'Alambertian and the parameters $\xi$ and $m^{2} . F_{0}, F_{1}$, and $F_{2}$ also depend on an energy scale $\mu$, introduced by the regularization method.

The dressed coupling constants depend on the energy scale $\mu$ according to the RG equations. Using minimal substraction these equations read 11

$$
\begin{aligned}
& \mu \frac{d G}{d \mu}=\frac{G^{2} m^{2}}{\pi}\left(\xi-\frac{1}{6}\right), \\
& \mu \frac{d \alpha}{d \mu}=-\frac{1}{32 \pi^{2}}\left[\left(\frac{1}{6}-\xi\right)^{2}-\frac{1}{90}\right], \\
& \mu \frac{d \beta}{d \mu}=-\frac{1}{960 \pi^{2}} .
\end{aligned}
$$

The dependence of $F_{0}, F_{1}$ and $F_{2}$ on $\mu$ is such that the full equation is $\mu$-independent. For example, from Eqs. (IX.8) and (IX.9), we see that $F_{0}=m^{2} \ln \left(\frac{m^{2}}{\mu^{2}}\right)\left(\xi-\frac{1}{6}\right)+$ const.

When the scalar field is massless, this information is enough to fix completely the form factors. Indeed, as the $F_{i}, i=1,2$ are dimensionless two point functions, by simple dimensional analysis we obtain $F_{i}\left(\square, \mu^{2}, \xi\right)=$ $F_{i}\left(\frac{\square}{\mu^{2}}, \xi\right)$. Inserting this into Eq. ([X.8), using Eqs. (IX.10) and ([X.11), and the fact that $S_{\text {eff }}$ must be independent of $\mu$, we obtain

$$
\begin{aligned}
& F_{1}(\square)=\frac{1}{2}\left[\left(\xi-\frac{1}{6}\right)^{2}-\frac{1}{90}\right] \ln \left[\frac{-\square}{\mu^{2}}\right]+\text { const }, \\
& F_{2}(\square)=\frac{1}{60} \ln \left[\frac{-\square}{\mu^{2}}\right]+\text { const. }
\end{aligned}
$$

The final result for the effective action has a clear interpretation: it is just the classical action in which the coupling constants $\alpha$ and $\beta$ have been replaced by nonlocal two point functions that take into account their running in configuration space.

For a massive field, the situation is more complex because there is an additional dimensional parameter. The form factors also depend on $\frac{m^{2}}{\mu^{2}}$ and the $\mu$-independence of the effective action is not enough to fix the form of them. They have already been computed in the literature 88, 89. 


$$
F_{i}(\square)=\int_{0}^{1} d \gamma \chi_{i}(\xi, \gamma) \ln \left[\frac{m^{2}-\frac{1}{4}\left(1-\gamma^{2}\right) \square}{\mu^{2}}\right]
$$

where

$$
\begin{aligned}
& \chi_{1}(\xi, \gamma)=\frac{1}{2}\left[\xi^{2}-\frac{1}{2} \xi\left(1-\gamma^{2}\right)+\frac{1}{48}\left(3-6 \gamma^{2}-\gamma^{4}\right)\right], \\
& \chi_{2}(\xi, \gamma)=\frac{1}{12} \gamma^{4} .
\end{aligned}
$$

These equations can be obtained through a covariant perturbation expansion 88, or by a resummation of the Schwinger DeWitt expansion [89]. Of course these form factors coincide with our previous Eq. (IX.12) in the massless case. For the sake of completness we will consider in what follows the massive case, although it is clear that only in the massless case the form factors are determined by the RG equations.

In order to clarify the meaning of the two point functions appearing in Eqs. (IX.12) and (IX.13), it is useful to introduce the following integral representation

$$
\ln \left[\frac{m^{2}-\frac{1}{4}\left(1-\gamma^{2}\right) \square}{\mu^{2}}\right]=\left[\ln \frac{\left(1-\gamma^{2}\right)}{4}+\int_{0}^{\infty} d z\left(\frac{1}{z+\mu^{2}}-G_{E}^{(z)}\right)\right],
$$

so the logarithm of the d'Alambertian is written in terms of the massive Euclidean propagator $G_{E}^{(z)}=$ $\left(z+\frac{4 m^{2}}{\left(1-\gamma^{2}\right)}-\square\right)^{-1}$. This representation will also be useful to construct the CTP version of the effective action.

Up to here we considered the Euclidean effective action. What about the in-out and CTP CGEA? Of course one can compute them from first principles using the covariant expansion, and indeed there are some calculations in the literature for the CTP CGEA [90]. However, in order to emphazise the relation with the RG equations, we will construct the CTP CGEA from its Euclidean counterpart.

Replacing the Euclidean propagator by the Feynman one in the integral representation Eq. (IX.15), one obtains the usual in-out effective action. As we already pointed out, the effective equations derived from this action are neither real nor causal because they are equations for in-out matrix elements and not for mean values.

The CTP CGEA can be written as

$$
e^{i S_{e f f}\left[g^{+}, g^{-}\right]}=N e^{i\left(S_{\text {grav }}\left[g^{+}\right]-S_{\text {grav }}\left[g^{-}\right]\right)} \int \mathcal{D} \phi^{+} \mathcal{D} \phi^{-} e^{i\left(S_{\text {matter }}\left[g^{+}, \phi^{+}\right]-S_{\text {matter }}\left[g^{-}, \phi^{-}\right]\right)},
$$

and the field equations are obtained from taking the variation of this action with respect to the $g_{\mu \nu}^{+}$metric, and then setting $g_{\mu \nu}^{+}=g_{\mu \nu}^{-}$.

In an alternative, and more concise notation, we can write this effective action as [91]

$$
e^{i S_{\text {eff }}^{\mathcal{C}}[g]}=N e^{i S_{\text {grav }}^{\mathcal{C}}[g]} \int \mathcal{D} \phi e^{i S_{\text {matter }}^{\mathcal{C}}[g, \phi]},
$$

where we have introduced the CTP complex temporal path $\mathcal{C}$, going from minus to plus infinity $\mathcal{C}_{+}$and backwards $\mathcal{C}_{-}$, with a decreasing (infinitesimal) imaginary part. Time integration over the contour $\mathcal{C}$ is defined by $\int_{\mathcal{C}} d t=\int_{\mathcal{C}_{+}} d t-\int_{\mathcal{C}_{-}} d t$. The field $\phi$ appearing in Eq. (IX.17) is related to those in Eq. (IX.16) by $\phi(t, \vec{x})=\phi_{ \pm}(t, \vec{x})$ if $t$ belongs to $\mathcal{C}_{ \pm}$. The same applies to $g_{\mu \nu}$.

This equation is useful because it has the structure of the usual in-out or the Euclidean effective action. Feynman rules are therefore the ordinary ones, replacing the Euclidean propagator by

$$
G(x, y)= \begin{cases}G_{F}(x, y)=i\langle 0, i n|T \phi(x) \phi(y)| 0, i n\rangle, & t, t^{\prime} \text { both on } \mathcal{C}_{+} \\ G_{D}(x, y)=-i\langle 0, i n|\tilde{T} \phi(x) \phi(y)| 0, i n\rangle, & t, t^{\prime} \text { both on } \mathcal{C}_{-} \\ G_{+}(x, y)=-i\langle 0, i n|\phi(x) \phi(y)| 0, i n\rangle, & t \text { on } \mathcal{C}_{-}, t^{\prime} \text { on } \mathcal{C}_{+} \\ G_{-}(x, y)=i\langle 0, i n|\phi(y) \phi(x)| 0, i n\rangle, & t \text { on } \mathcal{C}_{+}, t^{\prime} \text { on } \mathcal{C}_{-}\end{cases}
$$

Introducing Riemann normal coordinates, we can write, up to lowest order in the curvature

$$
G_{F}(x, y)=\int \frac{d^{4} p}{(2 \pi)^{4}} \frac{e^{i p(x-y)}}{p^{2}+m^{2}-i \epsilon}=G_{D}^{*}(x, y)
$$




$$
G_{ \pm}(x, y)=\mp \int \frac{d^{4} p}{(2 \pi)^{4}} e^{i p(x-y)} 2 \pi i \delta\left(p^{2}-m^{2}\right) \theta\left( \pm p^{0}\right) .
$$

All of the preceeding formulation of the effective action is valid for any field theory. In our particular case, we must replace the Euclidean propagator $G_{E}^{(z)}$ in Eq. (IX.15) by the propagator $G(x, y)$ of Eq. (IX.18) with a mass given by $\frac{4 m^{2}}{1-\gamma^{2}}+z$. After integration in $z$ we obtain

$$
\ln \left[\frac{\frac{4 m^{2}}{\left(1-\gamma^{2}\right)}-\square}{\mu^{2}}\right]_{C T P}= \begin{cases}\int \frac{d^{4} p}{(2 \pi)^{4}} e^{i p(x-y)} \ln \left(\frac{\left(1-\gamma^{2}\right)\left(p^{2}-i \epsilon\right)+4 m^{2}}{\mu^{2}}\right) & t, t^{\prime} \text { both on } \mathcal{C}_{+} \\ \int \frac{d^{4} p}{(2 \pi)^{4}} e^{i p(x-y)} \ln \left(\frac{\left(1-\gamma^{2}\right)\left(p^{2}+i \epsilon\right)+4 m^{2}}{\mu^{2}}\right) & t, t^{\prime} \text { both on } \mathcal{C}_{-} \\ \int \frac{d^{4} p}{(2 \pi)^{4}} e^{i p(x-y)} 2 \pi i \theta\left(p^{0}\right) \theta\left(-p^{2}-\frac{4 m^{2}}{1-\gamma^{2}}\right) & t \text { on } \mathcal{C}_{-}, t^{\prime} \text { on } \mathcal{C}_{+} \\ -\int \frac{d^{4} p}{(2 \pi)^{4}} e^{i p(x-y)} 2 \pi i \theta\left(-p^{0}\right) \theta\left(-p^{2}-\frac{4 m^{2}}{1-\gamma^{2}}\right) & t \text { on } \mathcal{C}_{+}, t^{\prime} \text { on } \mathcal{C}_{-}\end{cases}
$$

With the expression for the CTP logarithm of the d'Alambertian we can calculate explicitly the CTP effective action. Using the previous notation with $g_{\mu \nu}^{+}$and $g_{\mu \nu}^{-}$the CTP effective action reads

$$
\begin{aligned}
& S_{e f f}\left[g^{+}, g^{-}\right]=S_{\text {grav }}^{r}\left[g^{+}\right]-S_{\text {grav }}^{r}\left[g^{-}\right] \\
& \quad+\frac{i}{8 \pi^{2}} \int d^{4} x \int d^{4} y \Delta(x) \Delta(y) N_{1}(x, y)-\frac{1}{8 \pi^{2}} \int d^{4} x \int d^{4} y \Delta(x) \Sigma(y) D_{1}(x, y) \\
& \quad+\frac{i}{8 \pi^{2}} \int d^{4} x \int d^{4} y \Delta_{\mu \nu}(x) \Delta^{\mu \nu}(y) N_{2}(x, y)-\frac{1}{8 \pi^{2}} \int d^{4} x \int d^{4} y \Delta_{\mu \nu}(x) \Sigma^{\mu \nu}(y) D_{2}(x, y),
\end{aligned}
$$

where $\Delta=\frac{R^{+}-R^{-}}{2}, \Sigma=\frac{R^{+}+R^{-}}{2}, \Delta_{\mu \nu}=\frac{R_{\mu \nu}^{+}-R_{\mu \nu}^{-}}{2}, \Sigma_{\mu \nu}=\frac{R_{\mu \nu}^{+}+R_{\mu \nu}^{-}}{2}$. The classical gravitational action $S_{g r a v}^{r}$ contains the dressed, $\mu$-dependent coupling constants and we absorbed $F_{0}$ into the gravitational constant $G$.

The real and imaginary parts of $S_{\text {eff }}$ can be associated with the dissipation and noise, respectively. The dissipation $D_{i}$ and noise $N_{i}$ kernels are given by

$$
\begin{gathered}
D_{i}(x, y)=\int_{0}^{1} d \gamma \chi_{i}(\xi, \gamma) \int \frac{d^{4} p}{(2 \pi)^{4}} \cos [p(x-y)] \ln \left|\frac{\left(1-\gamma^{2}\right) p^{2}+4 m^{2}}{\mu^{2}}\right|, \\
N_{i}(x, y)=\int_{0}^{1} d \gamma \chi_{i}(\xi, \gamma) \int \frac{d^{4} p}{(2 \pi)^{4}} \cos [p(x-y)] \theta\left(-p^{2}-\frac{4 m^{2}}{1-\gamma^{2}}\right) .
\end{gathered}
$$

It is important to note that the imaginary part of this effective action must be positive definite. To make this point explicit, one can write the imaginary part in terms of the Weyl tensor $C_{\mu \nu \alpha \beta}$ and the scalar curvature $R$ by means of the following relation: $C_{\mu \nu \alpha \beta} C^{\mu \nu \alpha \beta}=2 R_{\mu \nu} R^{\mu \nu}-2 / 3 R^{2}$. It is not difficult to show that the scalar and tensor contributions to the imaginary part of the effective action are both positive.

In order to derive the ELEs we proceed as in Section VII B. One can regard the imaginary part of the CTP CGEA as coming from two classical stochastic sources $\eta(x)$ and $\eta^{\mu \nu \alpha \beta}(x)$, where the last tensor has the symmetries of the Weyl tensor. In fact, we can write the imaginary part as

$$
\begin{aligned}
\int \mathcal{D} \eta(x) & \int \mathcal{D} \eta^{\mu \nu \alpha \beta}(x) P\left[\eta, \eta^{\mu \nu \alpha \beta}\right] \exp \left(i\left\{\Delta(x) \eta(x)+\Delta_{\mu \nu \alpha \beta} \eta^{\mu \nu \alpha \beta}\right\}\right) \\
= & \exp \left\{-\int d^{4} x \int d^{4} y\left[\Delta(x) \tilde{N}(x-y) \Delta(y)+\Delta_{\mu \nu \alpha \beta}(x) N_{2}(x-y) \Delta^{\mu \nu \alpha \beta}(y)\right]\right\},
\end{aligned}
$$

where $\tilde{N}(x, y)=N_{1}(x, y)+\frac{1}{3} N_{2}(x, y)$, and $\Delta_{\mu \nu \alpha \beta}=\frac{1}{2} C_{\mu \nu \alpha \beta}^{+}-C_{\mu \nu \alpha \beta}^{-}$. The Gaussian functional probability distribution $P\left[\eta, \eta^{\mu \nu \alpha \beta}\right]$ is given by

$$
\begin{aligned}
P\left[\eta, \eta^{\mu \nu \alpha \beta}\right]=A & \exp \left\{-\frac{1}{2} \int d^{4} x \int d^{4} y \eta(x)[\tilde{N}(x, y)]^{-1} \eta(y)\right\} \\
& \times \exp \left\{-\frac{1}{2} \int d^{4} x \int d^{4} y \eta_{\mu \nu \alpha \beta}(x)\left[N_{2}(x, y)\right]^{-1} \eta^{\mu \nu \alpha \beta}(y)\right\},
\end{aligned}
$$


with $A$ a normalization factor.

Therefore, the CTP CGEA can be written as

$$
\exp \left\{i S_{e f f}\right\}=\int \mathcal{D} \eta \mathcal{D} \eta_{\mu \nu \alpha \beta} P\left[\eta, \eta_{\mu \nu \alpha \beta}\right] \exp \left\{i A_{e f f}\left[\Delta, \Delta_{\mu \nu \alpha \beta}, \Sigma, \Sigma_{\mu \nu}, \eta, \eta_{\mu \nu \alpha \beta}\right]\right\},
$$

where

$$
A_{e f f}=R e S_{e f f}+\int d^{4} x\left[\Delta(x) \eta(x)+\Delta_{\mu \nu \alpha \beta}(x) \eta^{\mu \nu \alpha \beta}\right] .
$$

The field equations $\left.\frac{\delta A_{e f f}}{\delta g_{\mu \nu}^{+}}\right|_{g_{\mu \nu}^{+}=g_{\mu \nu}^{-}}=0$, the Einstein-Langevin equations, are

$$
\begin{aligned}
& \frac{1}{8 \pi G}\left(R^{\mu \nu}-\frac{1}{2} g^{\mu \nu} R\right)-\tilde{\alpha} H_{\mu \nu}^{(1)}-\tilde{\beta} H_{\mu \nu}^{(2)} \\
& =-\frac{1}{32 \pi^{2}} \int d^{4} y D_{1}(x, y) H_{\mu \nu}^{(1)}(y)-\frac{1}{32 \pi^{2}} \int d^{4} y D_{2}(x, y) H_{\mu \nu}^{(2)}(y) \\
& +g^{\mu \nu} \square \eta-\eta^{; \mu \nu}+2 \eta_{; \alpha \beta}^{\mu \alpha \nu \beta},
\end{aligned}
$$

where $\tilde{\alpha}$ and $\tilde{\beta}$ differ from $\alpha$ and $\beta$ by $\xi$-dependent finite constants. Eq. (IX.29) is the main result of this section. The r.h.s. consists of the mean value of the energy-momentum tensor for the scalar field plus a stochastic correction characterized by the two point correlation functions

$$
\begin{aligned}
<\eta(x) \eta(y)> & =\tilde{N}(x, y) \\
<\eta_{\mu \nu \alpha \beta}(x) \eta_{\rho \sigma \lambda \tau}(y)> & =T_{\mu \nu \alpha \beta \rho \sigma \lambda \tau} N_{2}(x, y),
\end{aligned}
$$

where the tensor $T_{\mu \nu \alpha \beta \rho \sigma \lambda \tau}$ is a linear combination of four-metric products in such a way that the r.h.s of Eq. (IX.30) keeps the Weyl's symmetries. The scalar-noise kernel is given by

$$
\tilde{N}(x, y)=\frac{1}{2} \int_{0}^{1} d \gamma\left[\left(\xi-\frac{\left(1-\gamma^{2}\right)}{4}\right)^{2}-\frac{\gamma^{4}}{36}\right] \int \frac{d^{4} p}{(2 \pi)^{4}} \cos [p(x-y)] \theta\left(-p^{2}-\frac{4 m^{2}}{1-\gamma^{2}}\right) .
$$

In the massless case $\tilde{N}$ is proportional to $(\xi-1 / 6)^{2}$, and vanishes for conformal coupling. Therefore this term is present when the quantum fields are massive and/or when the coupling is not conformal. This is to be expected, since the imaginary part of the CTP CGEA signifies particle creation. For massless, conformally coupled quantum fields, particle creation takes place only when the spacetime is not conformally flat. Therefore in this case the only contribution to the imaginary part of the CTP CGEA is proportional to the square of the Weyl tensor. When the fields are massive and/or non-conformally coupled, particle creation takes place even when the Weyl tensor vanishes. This is why an additional contribution proportional to $R^{2}$ appears in the imaginary part of the effective action.

From Eq. (IX.29) we can define the effective energy-momentum tensor

$$
T_{\mu \nu}^{e f f}=<T_{\mu \nu}>+T_{\mu \nu}^{s t o c h}=<T_{\mu \nu}>+g^{\mu \nu} \square \eta-\eta^{; \mu \nu}+2 \eta_{; \alpha \beta}^{\mu \alpha \nu \beta},
$$

where $\left\langle T_{\mu \nu}\right\rangle$ is the quantum expectation value of the energy- momentum tensor of the quantum field and $T_{\mu \nu}^{\text {stoch }}$ is the contribution of the stochastic force, which in turn has contributions from the scalar and tensor noises. In the massless-conformal case the scalar-noise kernel vanishes, and $\left(T_{\mu}{ }^{\mu}\right)^{\text {stoch }}=0$, because the noise-source $\eta^{\mu \nu \alpha \beta}$ has vanishing trace. This means that there is no stochastic correction to the trace anomaly [80].

To summarize, we have obtained the ELEs using a covariant expansion in powers of the curvature. Our results are valid for quantum scalar fields with arbitrary mass and coupling to the curvature $\xi$. In the massless case, still for arbitrary $\xi$, we have shown that it is possible to obtain the noise and dissipation kernels using only dimensional analysis, the running of the coupling constants and the relation between the Euclidean and CTP CGEA. From this point of view, we can therefore conclude that the RG equations already contain information about the dissipation and noise kernels.

The results of this section can be easily generalized to any field theory with massless quantum fields in gravitational or Yang Mills backgrounds. 


\section{RENORMALIZATION GROUP AND QUANTUM CORRECTIONS TO THE NEWTONIAN POTENTIAL}

Two major areas of application for the stochastic and semiclassical gravity programs are the study of the final states of black holes and the physics of the early Universe. These are enormously complicated problems. We will describe here one of the simplest applications of semiclassical gravity, the calculation of quantum corrections to the Newtonian potential. Although a very simple example, it will shed light on important conceptual issues, in particular on the relation between the quantum corrections and the renormalization group.

Duff [92 computed the corrections to the Newtonian potential produced by the vacuum polarization of gravitons. His result is, schematically,

$$
V_{e f f}(r)=-\frac{G M}{r}\left(1+a \frac{G}{r^{2}}\right)
$$

This result has been rederived more recently by a number of authors 93 95. It was also shown that corrections of this form are the leading quantum corrections for massless quantum fields. The constant $a$ depends on the number and spin of massless fields. The first derivation based in the SEE has been presented in Ref. 96]. Recently there is a renewed interest in this type of corrections since it is relevant to relating two different developments in quantum gravity: Maldacena's Ads/CFT correspondence and the RandallSundrum alternative to compactification, see for example [97].

There is an alternative, intuitive "Wilsonian" way of taking into account, at least partially, the quantum effects: just modify the classical potential by replacing the Newton constant by its running counterpart

$$
V(r)=-\frac{G\left(\mu=\frac{1}{r}\right) M}{r}
$$

where $G(\mu)$ is the solution to the renormalization group equations in the theory considered. As can be seen from Eqs. (IX.9) and (X.1) this argument does not reproduce the leading quantum correction for massless fields.

We will now show that the result $\mathrm{X} .1$ ) can be derived from the ELEs, and are therefore a consequence of the RG equations for the parameters $\alpha$ and $\beta$. For simplicity, and to make contact with previous works, we will solve the ELEs without including the noise source. Solutions to the ELEs including this source can be found in 98$]$.

In the static, weak field approximation $g_{\mu \nu}=\eta_{\mu \nu}+h_{\mu \nu}$, we have

$$
\begin{aligned}
R_{\mu \nu} & =-\frac{1}{2} \square h_{\mu \nu} \\
R & =-\frac{1}{2} \square h \\
H_{\mu \nu}^{(1)} & =\left(-2 \partial_{\mu} \partial_{\nu}+2 \eta_{\mu \nu} \square\right) \square h \\
H_{\mu \nu}^{(2)} & =\left(-\partial_{\mu} \partial_{\nu}+\frac{1}{2} \eta_{\mu \nu} \square\right) \square h+\square \square h_{\mu \nu},
\end{aligned}
$$

where $h=\eta^{\mu \nu} h_{\mu \nu}$ and we assumed the Lorentz gauge condition $\left(h_{\mu \nu}-\frac{1}{2} h \eta_{\mu \nu}\right)^{; \nu}=0$.

Including a point particle source with $T_{\mu \nu}=\delta_{\mu}^{0} \delta_{\nu}^{0} \delta^{3}(\mathbf{x})$, the linearized field equations become,

$$
\left[-\frac{1}{16 \pi G}+\frac{m^{2}}{32 \pi^{2}}\left(\xi-\frac{1}{6}\right) \ln \frac{m^{2}}{\mu^{2}}\right] \square \bar{h}_{\mu \nu}-\alpha H_{\mu \nu}^{(1)}-\beta H_{\mu \nu}^{(2)}=T_{\mu \nu}+\left\langle T_{\mu \nu}\right\rangle
$$

with $\bar{h}_{\mu \nu}=h_{\mu \nu}-\frac{1}{2} h \eta_{\mu \nu}$. We define the quantum corrected Newtonian potential as $V(r)=-\frac{1}{2} h_{00}$.

The trace of the field equations is

$$
\left[\frac{1}{16 \pi G}-\frac{m^{2}}{32 \pi^{2}}\left(\xi-\frac{1}{6}\right) \ln \frac{m^{2}}{\mu^{2}}\right] \nabla^{2} h-2(3 \alpha+\beta) \nabla^{2} \nabla^{2} h=T_{\mu}^{\mu}+\left\langle T_{\mu}^{\mu}\right\rangle,
$$

where, to first order in $\frac{m^{2}}{\nabla^{2}}$, 


$$
\left\langle T_{\mu}^{\mu}\right\rangle=-\frac{1}{32 \pi^{2}}\left[3\left(\xi-\frac{1}{6}\right)^{2} \ln \left(-\frac{\nabla^{2}}{\mu^{2}}\right) \nabla^{2} \nabla^{2}-6 m^{2}\left(\xi^{2}-\frac{1}{36}\right) \ln \left(-\frac{\nabla^{2}}{m^{2}}\right) \nabla^{2}\right] h .
$$

We shall solve Eq. (X.8) perturbatively $h=h^{(0)}+h^{(1)}$. The classical contribution $h^{0}$ satisfies

$$
\left(\nabla^{2}-\sigma^{-2} \nabla^{2} \nabla^{2}\right) h^{(0)}=-16 \pi G M \delta^{3}(\mathbf{x}) \quad \sigma^{-2}=32 \pi G(3 \alpha+\beta) .
$$

The time independent and spherically symmetric solution is

$$
h^{(0)}=\frac{4 G M}{r}\left(1-e^{-\sigma r}\right) .
$$

The equation for the first quantum correction is

$$
\left(\nabla^{2}-\sigma^{-2} \nabla^{2} \nabla^{2}\right) h^{(1)}=\mathcal{D}\left(\nabla^{2}\right) h^{(0)},
$$

where

$$
\mathcal{D}\left(\nabla^{2}\right)=-\frac{3 G}{2 \pi}\left(\xi-\frac{1}{6}\right)^{2} \ln \left(-\frac{\nabla^{2}}{\mu^{2}}\right) \nabla^{2} \nabla^{2}+\frac{G m^{2}}{\pi}\left[\frac{1}{2}\left(\xi-\frac{1}{6}\right) \ln \left(\frac{m^{2}}{\mu^{2}}\right)+3\left(\xi^{2}-\frac{1}{36}\right) \ln \left(-\frac{\nabla^{2}}{m^{2}}\right) \nabla^{2}\right] .
$$

To find a solution to this equation, we will consider the limit $\sigma r \rightarrow \infty$ (we are interested in long distance quantum corrections). In this limit $h^{(0)}=4 G M\left(\frac{1}{r}+4 \pi \sigma^{-2} \delta^{3}(\mathbf{x})\right)$ and the nonlocal operator in the rhs of Eq. X.12) can be easily evaluated. After a long calculation we obtain

$$
h^{(1)}=-\frac{24 G^{2} M m^{2}}{\pi}\left(\xi^{2}-\frac{1}{36}\right) \frac{\ln \frac{r}{r_{0}}}{r}-\frac{12 G^{2} M}{\pi}\left(\xi-\frac{1}{6}\right)^{2} \frac{1}{r^{3}}+\ldots
$$

The dots denote corrections at the origin, which are proportional to $\delta^{3}(\mathbf{x})$ and its derivatives. We have not included them because our quantum corrections are not accurate near the origin. Indeed, we have derived the modified Einstein equations under the assumptions $\nabla \nabla \mathcal{R} \gg \mathcal{R}^{2}$ and $m^{2} \mathcal{R} \ll \nabla \nabla R$. Both conditions are satisfied for the $\frac{G M}{r}$ potential if $G M \ll r \ll m^{-1}$, so the origin is excluded.

A similar analysis can be carried out for $\bar{h}_{00}$. We omit the details [99]. The final answer for the Newtonian potential is

$$
V(r)=-\frac{1}{2} h_{00}=-\frac{G M}{r}\left\{1+\frac{2 G}{\pi}\left[\frac{1}{2}\left(\xi-\frac{1}{6}\right)^{2}+\frac{1}{90}\right] \frac{1}{r^{2}}+\frac{2 m^{2} G}{\pi}\left(\xi^{2}+\frac{1}{12}\right) \ln \frac{r}{r_{0}}\right\} .
$$

From Eq. X.15) we see that there are two different terms in the quantum correction. The term containing the logarithm is qualitatively what we expected from 'Wilsonian' arguments (see Eq. X.2). However, the coefficient is not exactly the same as the one derived from the renormalization group equation (IX.9), unless $\xi=0$. Besides the running of $G$, we have obtained the leading quantum $r^{-3}$ correction, which is a consequence of the running of $\alpha$ and $\beta$ in configuration space.

\section{RENORMALIZATION GROUP THEORY FOR NONEQUILIBRIUM SYSTEMS}

\section{A. Summary Remarks}

We first give a schematic summary of this report and then discuss some general issues concerning RG theory for NEq systems.

The CGEA contains all the information about the influence of the environment on the system. The CTP version of the CGEA is suitable for the analysis of the dynamical evolution of the system under the influence of the environment, and takes into account effects of renormalization, dissipation and noise.

In this review we have described several applications of the in-out and in-in effective action in semiclassical gravity and cosmology, paying particular attention to the relation with the RG equations. The Euclidean averaged effective action is reviewed by Wetterich and his collaborators in an article in this conference.

We have described a perturbative calculation of the CTP CGEA in RW spacetimes. We have shown that the CTP CGEA is useful to derive the master and Langevin equations in quantum field theory. The 
imaginary part of the CTP CGEA produces diffusive terms in the master equation. These terms can induce the reduced density matrix to become diagonal as it evolves, and are therefore crucial for decoherence and quantum to classical transition of the system. We applied this formalism to investigate the decoherence of mean fields due to quantum fluctuations relevant for theories of structure formation in inflationary models.

We adapted the Wegner and Houghton Euclidean approach to the in-in CGEA in order to obtain an exact renormalization group equation for the dependence of $S_{\Lambda}$ on the coarse graining scale. The exact equation is extremely complicated. We have solved it using a derivative expansion. In this approximation, the CTP CGEA "decouples" [i.e. it is of the form $\left.S_{\Lambda}\left(\phi_{+}, \phi_{-}\right)=S_{\Lambda}\left(\phi_{+}\right)-S_{\Lambda}\left(\phi_{-}\right)\right]$and contains neither dissipative nor noise terms. Previous results on the RG improved effective potential are recovered under this approximation.

Efforts to find solutions beyond the adiabatic approximation are under investigation. We believe that this equation (or a simplified version of it) will play an essential role in the development of a renormalization group theory for nonequilibrium systems. We expect that, as soon as we decrease the scale from $\Lambda_{0}$, dissipative and noise terms will grow: the CGEA will develop an imaginary part (related to noise) and a real part containing interactions between the $\phi_{ \pm}$fields (dissipation). This can be easily checked both in the one loop approximation and from the exact RGE. Indeed, we have seen that the one loop CTP CGEA is in general non-real. On the other hand, the real and imaginary parts of the CGEA are not decoupled in Eq. (VIII.11), and a non-vanishing real part at $\Lambda=\Lambda_{0}$ will induce an imaginary part at lower scales.

One should be able to find a non-perturbative, $\Lambda$-dependent fluctuation-dissipation relation and RG equations for the coupling constants of the theory that include the noise effects.

Finally, in the context of semiclassical gravity we described an interesting relation between the usual RG equations (running coupling constants) and the CTP CGEA. We have seen that one can take into account backreaction effects of quantum fields on the spacetime metric using a "Wilsonian" effective action in which the parameters of the theory are replaced by their running counterparts (this form of the effective action suggests itself by demanding the theory to be independent of the scale $\mu$ introduced by dimensional regularization). As the running is local in momentum space, it becomes nonlocal in configuration space, and thus one obtains an Euclidean, nonlocal effective action for the spacetime metric previously obtained by using different approaches. This Euclidean action can be transformed into the CTP CGEA taking into account formal analogies between their definitions. The final result, which contains dissipation and noise effects, can be viewed as a consequence of the running couplings of the theory.

\section{B. Towards a Nonequilibrium Renormalization Group Theory}

There are three important aspects in the construction of a renormalization group theory for nonequilibrium systems: a) The idea behind the introduction of the renormalization group (RG) description of critical phenomena characterized by the running of the interaction parameters of the theory; b) The scale characteristic of the dynamical interaction absent in static critical phenomena; c) How noise and dissipation in the stochastic equations describing the effective dynamics are reflected in the renormalization group equations governing the parameters of the theory. Point a) is discussed in all theories of static critical phenomena. We approached this problem stressing the coarse graining and backreaction aspects. Point b) is discussed in dynamical critical phenomena (see e.g., [100]). We note, however, in the literature the noise in the dynamical Ginzburg-Landau equation is usually introduced by hand, and what is at issue is how the system behaves towards the critical point - neither is the origin of noise accounted for nor is the dissipative aspect of the system dynamics being incorporated in the flow. The first part of Point c), i.e., how dissipation and noise appear in an open system is discussed in this report. (For a discussion of how noise is identified in interacting quantum fields in both a prescribed open system and an effectively open system, see, [8,101].) The crucial remaining issues are how they affect the RG flow and how they manifest in the approach to critical points. We will make some general comments here, as research on nonequilibrium (NEq) RG is still in its infant stage, its application to self-organized criticality, driven-diffusive systems and turbulence notwithstanding.

It is perhaps helpful to reflect upon the basic ideas and procedures behind the introduction of the RGi.e., coarse graining and scaling. When a system has a certain degree of regularity (homogeneity, periodicity) depicted by some symmetry, one can choose to represent it with an equivalent coarse-grained description. Examples are decimation in real time RG applied to a lattice, where, e.g., in the Ising model every other spin is eliminated and the effective bond strength between remaining spins is doubled. This Kadanoff-Migdal procedure produces a new Hamiltonian, which, upon iteration, can transform an original system into a simpler 
one. In the momentum space description, this procedure transcribes (runs) the ultraviolet (short range) behavior of the system to its infrared (long-ranged) domain, and hence is useful for the description of critical phenomena, as the behavior of the system near the critical point is dominated by the appearance of long range order. Whether this procedure, which is one of many possible coarse-graining schemes, can faithfully depict or capture the essential physics depends on the compatibility of the procedure with the system and on the properties of the system near the critical point. If the coarse-graining respects the symmetry of the system, (e.g., for an anisotropic medium, use a different decimation grading in different directions commensurate with the symmetry of the medium) and if the system possesses some scaling properties near the critical point, then the transformed problem via the RG would preserve the same critical behavior as the original problem. Otherwise it fails. The applicability of the RG idea thus depends crucially on the choice of a coarse-graining procedure and the use of scaling concepts. Leaving scaling aside for now, which has more to do with the properties of the particular systems of interest than with the procedure for accessing relevant information about the system, our approach to nonequilibrium processes and NEqRG theory starts from examining closely the coarse-graining procedure.

\section{1. $R G$ procedures in the light of open system concepts}

As we stated clearly at the beginning of this report, the necessary steps to capture the essense of a physical system with a simplified depiction lies in : 1) distinguish the system from the environment; 2) coarse grain the environment and 3) measure how the coarse-grained environment influences the system in providing an effective kinematics or dynamics of the reduced system. Let us examine the RG procedure in the light of the open systems scheme of nonequilibrium statistical mechanics. The first step consists of a) separating the order parameter field $\Phi(x, t)$ into two parts, $\Phi=\Phi_{S}+\Phi_{E}$, where $\Phi_{S}$ and $\Phi_{E}$ are respectively our system and environment. We assume that $\Phi_{S}$ contains the lower $k$ wave modes and $\Phi_{E}$ the higher $k$ modes. In critical phenomena, $\Phi_{S}:|\vec{k}|<\Lambda / s, \quad \Phi_{E}: \Lambda / s<|\vec{k}|<\Lambda$. Here $\Lambda$ is the ultraviolet cutoff and $s>1$ is the coarse-graining parameter which gives the fraction of total $k$ modes counted in the environment. Then b) one 'integrates out' the short wavelength sector, which amounts to finding out the effect or backreaction of the coarse-grained environment (the short wavelength modes) on the system. This is most succinctly and forcefully executed by the use of coarse-grained effective action 顿, as illustrated in the examples of this report. The last step in the $\mathrm{RG}$ procedure is c) to introduce a rescaling of the $\mathrm{k}$-space and a rescaled field, e.g. $\vec{k}^{\prime}=s \vec{k}, \quad \phi_{S}^{\prime}\left(\vec{k}^{\prime}, t\right)=s^{-\frac{d+2}{2}} \phi_{S}(\vec{k}, t)$ (d being the spatial dimension). In identifying (or rather, demanding that they are equivalent, up to a certain order in the perturbation expansion) the new action in terms of the rescaled variables with the old action, one can obtain a set of differential RG, or the WegnerHoughton equation. At the critical point itself, this effective theory containing only the low frequency modes is insensitive to further coarse graining, and therefore is described by a fixed point of the RG flow.

For dynamical systems, in addition to the scaling transformation, an artificial device of the RG theory depicting the running of the coupling constants of the system towards the infrared region, we find a real time-dependence depicting the dynamics of the system.

Now that we have identified the steps of the RG procedure in parallel to the treatment of open systems we can ask a few questions to illuminate the relevant issues. It is easy to see where dissipation and noise occur in the open system. But the appearance or nonappearance of noise and dissipation in the RG equations for nonequilibrium dynamics is not a simple issue. That is why we deem it necessary to first provide a clear and thorough discussion of the conceptual and technical basis for addressing the origin and nature of noise and dissipation in nonequilibrium field theory, as this report attempts to do, before we move on to investigate the related issues in RG theory for nonequilibrium processes. When the RG procedure is viewed in the light of an open system theory - in effect the modes of interest in the system after the Kadanoff transformation form an open system, and the techniques of nonequilibrium field theory are the natural language to address these problems - the long wavelength / slow modes dynamics will display, in general, both noise and dissipation.

\footnotetext{
${ }^{8}$ In treating dynamical systems, we may also coarse grain the system according to some time scale, obtaining the effective theory for slow modes after coarse graining the fast modes. Sometimes what is slow compared to what is fast may not be taken at face value, but has to be determined relevant to the dynamics and symmetry of the system. (Viewing eternal inflation as 'static' and 'slow- roll' as dynamic is an example we showed.)
} 
Indeed, the possibility that a renormalization group flow may acquire stochastic features for nonequilibrium processes is even clearer if we think of the RG as encoding the process of eliminating irrelevant degrees of freedom from our description of a system 102]. These elimination processes lead as a rule to dissipation and noise, as was made manifest in the influence action and the CTP-CGEA approaches shown.

But why, one may ask, is it that we don't usually talk about dissipation in the system or noise in the environment in a RG theory. A simple answer is that RG running is different from real-time dynamics, and dissipation in the dynamics of the effective system does not show up as dissipation runs. Another simple answer is that the bulk of RG research has been focused on equilibrium, stationary properties rather than the nonequilibrium dynamics [103]. 9 A major task in NEqRG is to make explicit this point, i.e., where does dissipation in the open system (Langevin) dynamics show up in the RG equations? We can offer only some speculative observations:

\section{Stochastic RG Equations}

A) For apparently closed (yet effectively open) systems described by the Boltzmann dynamics, this issue is more involved. We learned that when the hierarchy of correlation functions are simply truncated (with no slaving) [101], the equation of motion for the finite set of low-order correlation functions is unitary, such as is the case in Vlasov dynamics, which is obviously non-equilibrium, yet no dissipation or noise appear as such. A similar case is the n-loop effective action in ordinary quantum field theory. If we view the separation between the classical background field and the quantum fluctuation field as between a system and an environment, then the ordinary n-loop effective action is only one special case of the coarse-grained effective action, with quantum fluctuations being integrated or coarse-grained away. This results in radiative corrections to the bare mass and charge as we learned from renormalization theory, which, in the nonequilibrium language, amounts to backreaction of the environment on the system. We would expect to see dissipation in the effective dynamics of the background field if we follow the general arguments of NEq field theory, but of course one never talks about dissipation or noise in the equations of motion derived from an n-loop effective action. So what is missing? The reason is similar for both cases: the mean field or background field is the lowest order of the hierarchy of correlation functions in the Schwinger-Dyson equations. Any loop expansion entails a truncation of the hierarchy and, just like Vlasov dynamics in classical mechanics, the equation of motion from the effective action is unitary. So again it is not just the dynamics which prompts the appearance of dissipation - the causal factorization condition which we call slaving (as in the molecular chaos assumption of Boltzmann) is responsible for it.

For example the circumstances whereby the ordinary RG equations are derived could be similar to the Vlasov or the loop approximation examples mentioned above, in that the correlation functions are truncated from the hierarchy with a factorization condition (rather than from slaving, where the correlation noise arises). This results in the coupling constants being modified only partially, in a way similar to the renormalized mass or charge in one-loop (quantum) field theory or the (classical) Vlasov dynamics following the averaged potential which replaces the particle interactions. In other words, the backreaction of the coarse-grained modes is not taken into account fully. We see this, e.g. for the $\phi^{4}$ theory: the rescaled theory differs from the original theory in the $\phi^{6}$ and higher order terms. By identifying the two theories in such a way prescribed by the ordinary RG theory procedure, we assumed that the error of ignoring the higher order corrections are inmaterial in our range of consideration. As such it is a good description of the theory only for systems which scale near the critical point, because there the higher order correlation functions can be ignored or incorporated in a way simply related to that of the lower order ones. Away from the critical point this is not true and this procedure would give bad results. That is the criterion whereby one can assert the equivalence of the RG transformed theory and the original one.

Thinking about the relation between the general nonequilibrium dynamics of an open system and that depicted by the RG transformation in this light, we can see that the former (say, starting from a nonlocal Langevin equation governing the system arising from the backreaction of another subsystem or environment

\footnotetext{
${ }^{9}$ As for whether it has to do with equilibrium versus nonequilibrium conditions, we know that even under equilibrium conditions there is always noise and dissipation, as can be seen from linear reponse theory. They are related by the fluctuation-dissipation theorem. Indeed it is the precise balance of these processes which sustains the equilibrium.
} 
via a Zwangzig-Mori projection operator) keeps track of much more information about the environment than that in the RG treatment of the critical regime (as slaving being more than truncation). Although this is not sufficient for a proper description of the effective dynamics as dissipation and fluctuations carry important information about the system and the environment, it is sufficient for the purpose of depicting the (static) critical phenomena of the system in the critical region, as scaling behavior simplifies the relation between the correlation functions. That is why in actual RG calculations one can assume that the cutoff is in effect infinitely far above the scales of interest. This advantage near the critical point will still come to the aid of dynamical critical phenomena, but when the full dynamics goes beyond an approach to the equilibrium (which is manifest in say, critical slowing down) or when one is interested in the behavior of the system farther away from the critical point, then more of the dissipative and noise attributes which are always present in general circumstances will have to be included in a nonequilibrium RG theory. The art in the design of such a theory for NEq processes is to find out what additional information (noise and dissipation) need be kept, or what degree of coarse-graining is sufficient for the RG procedure which can still capture the essence of the critical behavior for dynamical systems.

B) One place where one can indeed see the 'disappearance' of the effects of noise from the RG equations is when an adiabatic or quasilocal approximation is introduced on the mode function dynamics. In [52] Dalvit and Mazzitelli show from the Wegener - Houghton equation derived from the CTP effective action that, for static long wavelength fields, the usual RG is recovered. In view of this result it is clear that an understanding of the running of noise and dissipation requires consideration of dynamical long wavelength fields. However, if these fields are slowly varying, they may be considered as actually linear or quadratic in the space - time coordinates, in which case the functional integration of the high momentum modes may be carried out exactly, at least to one loop order [26,104.

Alternatively, we may consider that the low momentum modes are following a prescribed, though not necessarily slow, evolution, and compute the back reaction of the high momentum modes keeping track of non adiabatic effects. Such an analysis is carried out in [105] (See also [53]), where the fast modes are represented by a massive heavy field interacting with slow modes, described as a light field. As expected, noise and dissipation display significant running (they increase exponentially), as the gap between the characteristic frequencies of the heavy and light fields narrows. This result suggests that a nonequilibrium RG should include, besides the usual interactions, the running of suitable parameters describing both noise and dissipation.

Therefore the challenge is to construct a RG flow where the dissipative and noisy characteristics of the dynamics is ingrained therein. There is a precedent for this problem in studies of RG flow in the Navier - Stokes equation [106], where one is interested, for example, in the running of the viscosity. In this kind of research, the starting point is generally a Langevin type equation, whose solutions are given through functional representations following the methods of Zinn-Justin, e.g., 100 (see also [107]). We would like to point out that even in this context, closed time - path techniques offer an alternative way to obtain a functional representation of the solutions of the Langevin equation [108], which is in several aspects simpler than the more familiar, forward time path, one.

C) Another lead can be found in the stochastic correlation function $\mathbf{G}$ introduced in a quantum field theoretical derivation of the Boltzmann-Langevin equation [101]. Its expectation value reproduces the usual propagators (Green functions), while its fluctuations account for the quantum fluctuations in the binary product of (operator) fields. The dynamical equation for $\mathbf{G}$ takes the form of an explicitly stochastic Dyson equation. In the kinetic limit, the fluctuations in $\mathbf{G}$ become the classical fluctuations in the one particle distribution function, and the dynamical equation for G's Wigner transform becomes the Boltzmann Langevin equation. (Each of these results has an interest of its own. A priori, there is no simple reason why the fluctuations derived from quantum field theory should have a physical meaning corresponding to a phenomenological entropy flux and Einstein's relation.) The studies of the fluctuating character of these field theoretic Green functions also suggest new avenues in the development of RG theory. For example, we are used to fixing the ambiguities of renormalization theory by demanding certain Green functions to take on given values under certain conditions (conditions which should resemble the physical situation of interest as much as possible, as stressed by O'Connor and Stephens 109]). If the Green functions themselves are to be regarded as fluctuating, then the same ought to hold for the renormalized coupling constants defined from them, and for the renormalization group (RG) equations describing their scale dependence. The notion that Green functions (and indeed, higher correlations as well) may or even ought to be seen as possessing fluctuating characters (when placed in the larger context of the whole hierarchy) with clearly discernable physical meanings is likely to have an impact on the way we perceive the statistical properties of field theory. 
To end, we note that while the application of renormalization group methods to stochastic equations is presented in well-known monographs 100], our proposal here goes beyond these results in at least two ways. First, in our approach the noise is not put in by hand or brought in from outside (e.g., the environment of an open system), as in the usual Langevin equation approach, but it follows from the (quantum) dynamics of the system itself. Actually, the possibility of learning about the system from the noise properties (whether it is white or coloured, additive or multiplicative, etc.) - unraveling the noise, or treating noise creativelyis a subtext in our program. Second, our result suggests that stochasticity may, or should, not only appear at the level of equations of motion, but also the level of the RG equations, as they describe the running of 'constants' which are themselves fluctuating. This we feel is the first task in the construction of a RG theory for NEq processes.

Acknowledgements We wish to thank the organizers of the RG2000 meeting in Taxco (Mexico), January 1999 for their warm hospitality, especially Denjoe O'Connor and Chris Stephens, with whom we enjoyed many close discussions over the years. We also enjoy the exchanges with David Huse, Michael Fisher and Jean Zinn-Justin during the meeting on the role of noise in nonequilibrium renormalization group theory. EC, FDM are supported in part by CONICET, UBA, Fundación Antorchas and Agencia Nacional de Promoción Científica y Tecnológica. BLH is supported in part by NSF grant PHY98-00967 and their collaboration is supported in part by NSF grant INT95-09847.

[1] E. Calzetta and B. L. Hu, Phys. Rev. D35, 495 (1987).

[2] E. Calzetta and B. L. Hu, Phys. Rev. D40, 656 (1989).

[3] B. L. Hu, Physica A158, 399 (1989).

[4] B. L. Hu and Y. Zhang, "Coarse-Graining, Scaling, and Inflation" Univ. Maryland Preprint 90-186; B. L. Hu, in Relativity and Gravitation: Classical and Quantum Proc. SILARG VII, Cocoyoc, Mexico 1990. eds. J. C. D' Olivo et al (World Scientific, Singapore 1991)

[5] B. L. Hu, Class.Quant.Grav. 10 (1993) S93

[6] B. L. Hu, J. P. Paz and Y. Zhang, "Quantum Origins of Noise and Fluctuations in Cosmology" in The Origins of Structures in the Universe" ed. E. Gunzig and P. Nardone (NATO ASI Series) (Plenum Press, New York, 1993) p. 227

[7] E. Calzetta and B. L. Hu, Phys. Rev. D 49, 6636 (1994)

[8] B. L. Hu, in Proceedings of the Third International Workshop on Thermal Fields and its Applications, CNRS Summer Institute, Banff, August 1993, edited by R. Kobes and G. Kunstatter (World Scientific, Singapore, 1994).

[9] M. W. Choptuik, Phys. Rev. Lett. 70, 9 (1993)

[10] H. J. de Vega, N. Sanchez, F. Combes, Phys. Rev. D54, 6008-6020 (1996 )

[11] I.L. Buchbinder, S.D. Odintsov and I.L. Shapiro in Effective action in quantum gravity, ( IOP Publishing Ltd, London, 1992)

[12] K. Kirsten, G. Guido and L. Vanzo, Phys. Rev. D 48, 2813 (1993),

[13] A. Bonanno, Phys. Rev. D 52, 9697 (1995).

[14] J. Ambjorn, Nucl. Phys. B, Proc. Suppl. 42 (1995) 3-16. In *Bielefeld 1994, Lattice '94* 3-16. hep-lat 9412006.

[15] I. Antoniadis, P. Mazur and E. Mottola, Phys. Lett. B 323 (1994) 284

[16] See, e.g., P. C. Hohenberg and B. I. Halperin, Rev. Mod. Phys. 49, 435 (1977). Ch. XII of S. K. Ma, Modern Critical Phenomena (Benjamin, N.Y. 1976); K. Kawasaki and J. Guntun, Phys. Rev. B13, 4658 (1976).

[17] J. M. Cornwall and R. Bruinsma, Phys. Rev. D38, 3146 (1988)

[18] B. L. Hu and A. Raval, in preparation

[19] R. M. Wald, Phys. Rev. D28, 2118 (1983)

[20] W. Boucher, G. W. Gibbons and G. T. Horowitz, Phys. Rev. D30, 2447 (1984)

[21] T. C. Shen, B. L. Hu and D. J. O'Connor, Phys. Rev. D31, 2401 (1985)

[22] F. Lucchin and S. Matarrese, Phys. Rev. D32, 1316 (198

[23] L. Kadanoff, Ann. Phys. (N.Y.) 2, 263 (1966); A. A. Migdal, Sov. Phys.-JETP 69, 810; L457 (1975).

[24] B. L. Hu, Phys. Lett. 123B, 189 (1983); B. L. Hu and L. F. Chen, Phys. Lett. 160B, 36 (1985).

[25] S. Sinha and B. L. Hu, Phys. Rev. D38, 2423 (1989).

[26] B. L. Hu, and D. J. O'Connor, Phys. Rev. D30, 743 (1984). 
[27] I. Moss, D. J. Toms and A. Wright, Phys. Rev. D46, 1670 (1992)

[28] B. L. Hu, in Proc. CAP-NSERC Summer Institute in Theoretical Physics, (ed.) F. C. Khanna, H. Umezawa, G. Kunstatter and P. Lee (World Scientific, Singapore 1988) Vol II.; B. L. Hu and D. J. O'Connor, Phys. Rev. D36, 1701(1987). D. J. O'Connor, C. R. Stephens and B. L. Hu, Ann. Phys. (N.Y.) 190,310 (1990).

[29] L. Kadanoff, Ann. Phys. (N.Y.) 100,359 (1976); Rev. Mod. Phys. 49, 267 (1977); K. Wilson and J. Kogut, Phys. Rep.C12,75(1974); M. E. Fisher, Rev. Mod. Phys. 46, 597 (1974) and in Critical Phenomena, (ed.) F.J.W. Hahne (Springer, Berlin 1983); J. F Nicoll, T. S. Chang and H.E. Stanley, Phys. Rev. A13, 1251 (1976); P. Pfeuty and G. Toulouse, Introduction to the Renormalization Group and to Critical Phenomena (Wiley, N.Y. 1977). For a simple introduction, see K. Huang, Statistical Mechanics (J. Wiley, N.Y. 1989) Ch. 18; Bambi Hu, Phys. Rep. 91, 233 (1982).

[30] See, e.g., E. Brezin, J. C. Lee Guillou and J. Zinn-Justin in Phase transitions and Critical Phenomena, Vol. VI (Academic Press, N.Y. 1976); D. J. Amit, Field Theory, The Renormalization Group and Critical Phenomena, Second edition (World Scientific, Singapore, 1985).

[31] A. A. Starobinsky, in Field Theory, Quantum Gravity and Strings, ed. H. J. de Vega and N. Sanchez (Springer, Berlin 1986); J. M. Bardeen and G. J. Bublik, Class. Quant. Grav. 4, 573 (1987); S. J. Rey, Nucl. Phys. B284, 706(1987).

[32] E. Calzetta and B. L. Hu, Phys. Rev. D52, 6770 (1995)

[33] S. A. Ramsey, B. L. Hu, Phys. Rev. D56, 678-705 (1997). Erratum-ibid. D57, 3798 (1998).

[34] F.D. Mazzitelli, J.P. Paz and C. El Hasi, Phys. Rev. D40, 955 (1989).

[35] S. Sinha and B. L. Hu, Phys. Rev. D 44, 1028 (1991)

[36] E. Braaten, R. D. Pisarski, Phys. Rev. D45, 1827-1830 (1992).

[37] C. Wetterich, Nucl. Phys. B352, 529 (1991); Phys. Lett. B301, 90 (1993); Z. Phys. C60, 461 (1993).

[38] B. S. DeWitt, in Quantum Concepts in Space and Time, ed. R. Penrose and C. J. Isham (Claredon Press, Oxford, 1986).

[39] R. D. Jordan, Phys. Rev. D33, 44 (1986).

[40] F. J. Wegner and A. Houghton, Phys. Rev. A8, 401 (1973)

[41] An interesting discussion of how this consideration motivated the use of scaling ideas and renormalization group techniques for the investigation of critical phenomena can be found in K. Wilson, Rev. Mod. Phys. 55, 583(1982). See also S. K. Ma, Modern Critical Phenomena (Benjamin, N.Y.1976).

[42] C. R. Stephens, private communication.

[43] J.M. Bardeen, P.J. Steinhardt and M.S.Turner, Phys. Rev. D28, 629(1983); A. Guth and S.Y Pi, Phys. Rev. Lett. 49, 1110 (1982);A.A. Starobinsky, Phys. Lett. 117B, 175(1982); S.W Hawking, Phys. Lett. 115B, 295(1982).

[44] T. A. Jacobson, Phys. Rev. D44, 1731 (1991).

[45] R. Feynman and F. Vernon, Ann. Phys. (NY) 24, 118 (1963). R. Feynman and A. Hibbs, Quantum Mechanics and Path Integrals, (McGraw - Hill, New York, 1965). H. Kleinert. Path Integrals in Quantum Mechanics, Statistics, and Polymer Physics (World Scientific, Singapore, 1990).

[46] A. O. Caldeira and A. J. Leggett, Physica 121A, 587 (1983); Ann. Phys. (NY) 149, 374 (1983).

[47] Y. Zhang, Ph. D. Thesis, University of Maryland, 1991 (unplished)

[48] B. L. Hu and A. Matacz, Phys. Rev. D51, 1577 (1995)

[49] D. Koks, A. Matacz and B.L. Hu, Phys. REv D59, 5917 (1997)

[50] F. C. Lombardo and F. D. Mazzitelli, Phys. Rev. D 53, 2001 (1996).

[51] C. Greiner and B. Müller, Phys. Rev. D 55, 1026 (1997)

[52] D. A. R. Dalvit and F. D. Mazzitelli, Phys. Rev. D 54, 6338 (1996).

[53] D. Boyanovsky, M. D’Attanasio, H.J. de Vega, R. Holman, D.-S. Lee, Phys. Rev. D52, 6805-6827 (1995).

[54] J. P. Paz, Phys. Rev. D41, 1054 (1990); D42, 529 (1990)

[55] S. Weinberg, Phys. Lett. 83B, 339 (1979)

[56] B. L. Hu, J. P. Paz and Y. Zhang, Phys. Rev. D45, 2843 (1992) B. L. Hu, J. P. Paz and Y. Zhang, Phys. Rev. D47, 1576 (1993)

[57] H. Grabert, P. Schramm and G. L. Ingold, Phys. Rep. 168, 115 (1988).

[58] W.G. Unruh, W.H. Zurek, Phys.Rev.D40:1071,1989.

[59] Z. Su et al, Phys. Rev. B37, 9810 (1988)

[60] J. P. Paz, in The Physical Origin of Time Asymmetry, ed. by J. Halliwell, J. Perez Mercader and W. Zurek (Cambridge University Press, Cambridge, 1994)

[61] F.C. Lombardo, F.D. Mazzitelli and D. Monteoliva, Phys. Rev. D62, 045016 (2000).

[62] L. Davila Romero and J.P. Paz, Phys. Rev. A55, 4070 (1997)

[63] M. Abramowitz and I. Stegun (eds.) of Handbook of Mathematical Functions, Dover Publications (N. Y.) (1972)

[64] S. Habib and R. Laflamme, Phys. Rev. D42, 4056(1990)

[65] J. J. Halliwell, T. Yu, Phys.Rev.D53:2012-2019,1996. 
[66] S. Habib, Phys. Rev. D46, 2408 (1992)

[67] S. B. Liao and J. Polonyi, Ann. Phys. 222, 122 (1993).

[68] J. Polchinski, Nucl. Phys. B231, 269 (1984).

[69] A. Hasenfratz and P. Hasenfratz, Nucl. Phys. B270, 687 (1986).

[70] T. R. Morris, Int. J. Mod. Phys. A 9, 2411 (1994).

[71] M. Bonini, M.D'Attanasio and G. Marchesini, Nucl. Phys. B490, 441 (1993).

[72] T. R. Morris, Phys. Lett. B 334, 355 (1994).

[73] T. R. Morris, Phys. Lett. B 329, 241 (1994).

[74] R. Ball et al., Phys. Lett. B 347, 80 (1995); P. Haagensen et al., Phys. Lett. B 323, 330 (1994).

[75] J. Adams et al., Mod. Phys. Lett. A 10, 2367 (1995).

[76] I. Gel'fand and A. Yaglom, J. Math. Phys. 1, 48 (1960); S. Coleman, Aspects of Symmetry (Cambridge University Press, Cambridge, England, 1985).

[77] S. B. Liao, J. Polonyi and D. Xu, Phys. Rev. D 51, 748 (1995); S. B. Liao and S. Strickland, Phys. Rev. D 52, 3653 (1995); M. D’Attanasio and M. Pietroni, Nucl. Phys. 472, 711 (1996).

[78] N. D. Birrell and P. C. W. Davies, Quantum Fields in Curved Space (Cambridge University Press, London, 1982)

[79] A. Campos and E. Verdaguer, Phys. Rev. D 49, 1861 (1994)

[80] A. Campos and E. Verdaguer, Phys. Rev. D 53, 1927 (1996)

[81] B.L. Hu, Int. J. Theor. Phys. 38, 2987 (1999)

[82] B. L. Hu and A. Matacz, Phys. Rev. D 51, 1577 (1995)

[83] B. L. Hu and S. Sinha, Phys. Rev. D 51, 1587 (1995)

[84] A. Roura and E. Verdaguer, Phys. Rev. D 60, 107503 (1999).

[85] E. Calzetta, A. Roura and E. Verdaguer, quant-ph/0011097.

[86] F. C. Lombardo and F. D. Mazzitelli, Phys. Rev. D 55, 3889 (1997).

[87] G.A. Vilkovisky, in Quantum Theory of Gravity, edited by S.M. Christensen (Hilger, Bristol, 1984)

[88] A.O. Barvinsky and G.A. Vilkovisky, Nucl. Phys. B282, 163 (1987); B333, 471 (1990)

[89] I.G. Avramidi, Yad. Fiz. 49, 1185 (1989) [Sov. J. Nucl. Phys. 49, 735 (1989)]

[90] W. Tichy and E. E. Flanagan, Phys.Rev.D58, 124007 (1998).

[91] F. Cooper et al, Phys. Rev. D 50, 2848 (1994)

[92] M.J. Duff, Phys. Rev. D9, 1837 (1974).

[93] J. F. Donoghue, Phys. Rev. Lett. 72, 2996 (1994).

[94] H. Hamber and S. Liu, Phys. Lett. B357, 51 (1995).

[95] I. Muzinich and S. Vokos, Phys. Rev D52, 3472 (1995).

[96] D.A.R. Dalvit and F. Mazzitelli, Phys. Rev. D 50, 1001 (1994); See also Phys. Rev. D 52, 2577 (1995) and Phys. Rev. D 56, 7779 (1997).

[97] M. J. Duff and J. T. Liu, Phys. Rev. Lett. 85, 2052 (2000); E. Alvarez and F.D. Mazzitelli, hep-th 0010203, to appear in Phys. Lett. B (2001).

[98] R. Martín and E. Verdaguer, Int. J. Theor. Phys. 38, 3049 (1999); R. Martín and E. Verdaguer, Phys. Lett. B 465, 113 (1999); R. Martín and E. Verdaguer, Phys. Rev. D 60, 084008 (1999); R. Martín and E. Verdaguer, Phys. Rev. D 61, 124024 (2000).

[99] D. A. R. Dalvit, doctoral thesis, Univ. of Buenos Aires, hep-th/9807112

[100] J. Zinn - Justin, Field Theory and Critical Phenomena (Oxford University Press, Oxford, 1989); K. Kawasaki and J. Gunton, Phys. Rev. B13, 4658 (1976); D. Forster, D. Nelson and M. Stephen, Phys. Rev. A16, 732 (1977).

[101] E. Calzetta and B. L. Hu, Phys. Rev. D61 (2000) 025012

[102] S. K. Ma, Modern Theory of Critical Phenomena (Benjamin, London, 1976); J. Zinn-Justin, Statistical Field Theory (John Wiley, New York, 1989); E. Brezin, J. C. le Guillou and J. Zinn-Justin Field Theoretical Approach to Critical Phenomena in Phase Transitions and Critical Phenomena edited by C. Domb and M. S. Green (Academic Press, London, 1976); M. E. Fisher, Rev. Mod. Phys. 70, 653 (1998).

[103] P. Hohenberg and B. Halperin, Rev. Mod. Phys. 49, 435 (1977); J. Cardy, Scaling and Renormalization in Statistical Physics (Cambridge University Press, Cambridge, 1996); M. Peskin and D. Schroeder, Quantum Field Theory (Addison - Wesley, New York, 1995)

[104] J. Schwinger, Phys. Rev. 82, 664-679 (1951); M. Brown and M. Duff, Phys. Rev. D11, 2124 (1975)

[105] E. Calzetta and B. L. Hu, Phys. Rev. D55, 3536 (1997)

[106] U. Frisch, Turbulence, Cambridge University Press (1995); and references therein.

[107] D. Hochberg et al, J. Stat. Phys, 99, 903 (2000); D. Hochberg, C. Molina-Paris and M. Visser, cond-mat 0009424.

[108] J. J. Halliwell, Phys. Rev. D58, 105015 (1998).

[109] D. O'Connor and C. R. Stephens, Int. J. Mod. Phys. A9, 2805 (1994); Phys. Rev. Lett 72, 506 (1994); M. 
Van Eijck, D. O'Connor and C. R. Stephens, Int. J. Mod. Phys. A10, 3343 (1995); C. R. Stephens, preprint hep-th/9611062; F. Freire, M. Van Eijck, D. O'Connor and C. R. Stephens, preprint hep-th/9601165. 


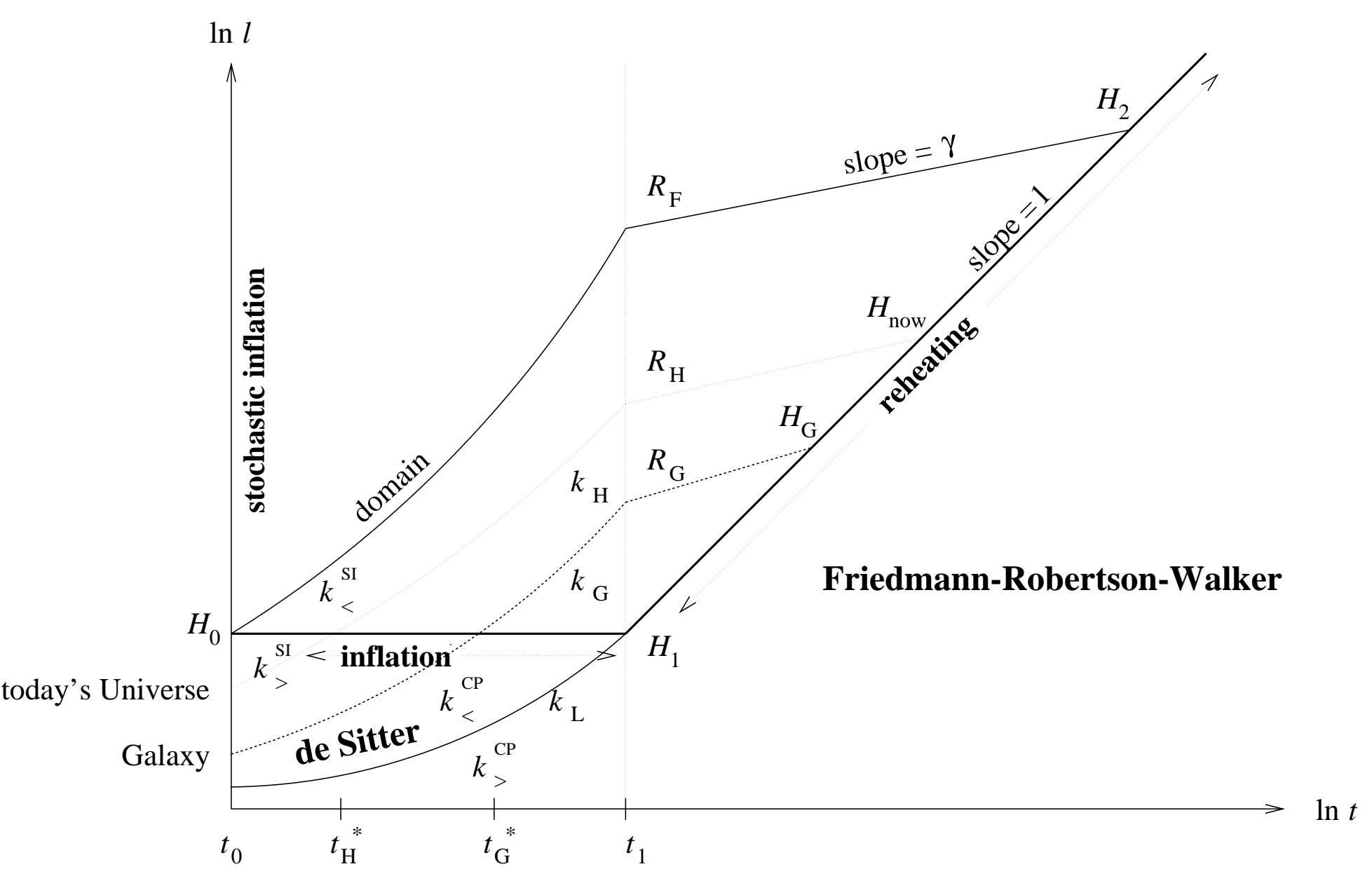


